Homology, Homotopy and Applications, vol.12(2), 2010, pp.167-210

\title{
ON THE SECOND COHOMOLOGY GROUP OF A SIMPLICIAL GROUP
}

\author{
SEBASTIAN THOMAS
}

(communicated by Graham Ellis)

\begin{abstract}
We give an algebraic proof for the result of EILENBERG and MAC LANE that the second cohomology group of a simplicial group $G$ can be computed as a quotient of a fibre product involving the first two homotopy groups and the first Postnikov invariant of $G$. Our main tool is the theory of crossed module extensions of groups.
\end{abstract}

\section{Introduction}

In [12], Eilenberg and Mac Lane assigned to an arcwise connected pointed topological space $X$ a topological invariant $k^{3} \in \mathrm{H}^{3}\left(\pi_{1}(X), \pi_{2}(X)\right)$, that is, a 3cohomology class of the fundamental group $\pi_{1}(X)$ with coefficients in the $\pi_{1}(X)$ module $\pi_{2}(X)$, which is nowadays known as the first Postnikov invariant of $X$. Thereafter, they showed that the second cohomology group of $X$ with coefficients in an abelian group $A$ only depends on $\pi_{1}(X), \pi_{2}(X)$ and $k^{3}$. Explicitly, they described this dependency as follows. We let $\operatorname{Ch}\left(\pi_{1}(X), A\right)$ denote the cochain complex of $\pi_{1}(X)$ with coefficients in $A$ and $\operatorname{Hom}_{\pi_{1}(X)}\left(\pi_{2}(X), A\right)$ denote the group of $\pi_{1}(X)$-equivariant group homomorphisms from $\pi_{2}(X)$ to $A$, where $\pi_{1}(X)$ is supposed to act trivially on A.

Theorem (Eilenberg, Mac Lane, 1946 [12, thm. 2]). We choose a 3-cocycle $z^{3} \in \mathrm{Z}^{3}\left(\pi_{1}(X), \pi_{2}(X)\right)$ such that $k^{3}=z^{3} \mathrm{~B}^{3}\left(\pi_{1}(X), \pi_{2}(X)\right)$. The second cohomology group $\mathrm{H}^{2}(X, A)$ is isomorphic to the quotient group

$$
Z^{2} / B^{2}
$$

where $Z^{2}$ is defined to be the fibre product of

$$
\mathrm{Ch}^{2}\left(\pi_{1}(X), A\right) \stackrel{\partial}{\longrightarrow} \mathrm{Ch}^{3}\left(\pi_{1}(X), A\right)
$$

Received November 15, 2009, revised May 11, 2010; published on September 20, 2010. 2000 Mathematics Subject Classification: 55U10, 18G30, 18D35, 20 J06.

Key words and phrases: cohomology, simplicial groups, crossed modules.

This article is available at http://intlpress.com/HHA/v12/n2/a6

Copyright (C) 2010, International Press. Permission to copy for private use granted. 
with vertical map given by $\varphi \mapsto z^{3} \varphi$, and where $B^{2}$ is defined to be the subgroup

$$
B^{2}:=\{0\} \times \mathrm{B}^{2}\left(\pi_{1}(X), A\right) \leqslant Z^{2} \leqslant \operatorname{Hom}_{\pi_{1}(X)}\left(\pi_{2}(X), A\right) \times \mathrm{Ch}^{2}\left(\pi_{1}(X), A\right) .
$$

In this article, we give an algebraic proof of the simplicial group version of the theorem of EILENBERG and MAC LANE, cf. theorem 5.4(b). Since simplicial groups are algebraic models for path connected homotopy types of CW-spaces, this yields an algebraic proof for their original theorem mentioned above.

It turns out to be convenient to work on the level of crossed modules. To any simplicial group $G$, we can attach its crossed module segment $\operatorname{Trunc}^{1} G$, while to any crossed module $V$, we can attach its simplicial group coskeleton $\operatorname{Cosk}_{1} V$. We have $\mathrm{H}^{2}(G, A) \cong \mathrm{H}^{2}\left(\operatorname{Cosk}_{1}\right.$ Trunc $\left.^{1} G, A\right)$. Moreover, the crossed module segment of $G$ suffices to define the Postnikov invariant $k^{3}$ of $G$ via choices of certain sections, see $[\mathbf{4}$, ch. IV, sec. 5] or [31, sec. 4]. These sections pervade our algebraic approach.

Related to this theorem, ELLIS [14, th. 10] has shown that there exists a long exact sequence involving the second cohomology group $\mathrm{H}^{2}(V, A)$ of a crossed module $V$ starting with

$$
0 \longrightarrow \mathrm{H}^{2}\left(\pi_{0}(V), A\right) \longrightarrow \mathrm{H}^{2}(V, A) \longrightarrow \operatorname{Hom}_{\pi_{0}(V)}\left(\pi_{1}(V), A\right)
$$

This part of his sequence is also a consequence of our EILENBERG-MAC LANE-type description of $\mathrm{H}^{2}(V, A)$, cf. theorem 5.4. $\left(^{1}\right)$

Concerning Postnikov invariants, cf. also [8], where general Postnikov invariants for crossed complexes, which are generalisations of crossed modules, are constructed.

\section{Outline}

In section 2, we recall some basic facts from simplicial algebraic topology, in particular cohomology of simplicial groups. We will recall how simplicial groups, crossed modules and (ordinary) groups interrelate. Finally, we will give a brief outline how a cohomology class can be attached to a crossed module - and hence to a simplicial group - and conversely.

In section 3, we will consider the low-dimensional cohomology groups of a simplicial group. The aim of this section is to give algebraic proofs of the well-known facts that the first cohomology group depends only on the group segment and the second cohomology group depends only on the crossed module segment of the given simplicial group. This gives already a convenient description of simplicial group cohomology in dimensions 0 and 1 , and can be seen in dimension 2 as a reduction step allowing us to work with crossed modules in the following.

In section 4, we introduce a certain standardised form of 2-cocycles and 2-coboundaries of a crossed module, which suffices to compute the second cohomology group. On the other hand, this standardisation directly yields the groups $Z^{2}$ and $B^{2}$ occurring in the description of EILENBERG and MAC LANE.

We apply our results of sections 3 and 4 in section 5 to simplicial groups, thus obtaining the analogon of EILENBERGs and MAC LANEs theorem. Finally, we discuss some corollaries and examples.

\footnotetext{
${ }^{1}$ Our notation here differs from ELLIS' by a dimension shift.
} 


\section{Acknowledgement}

I thank MATTHIAS KÜNZER for many useful discussions on this article and for directing me to the article of Eilenberg and Mac Lane [12].

\section{Conventions and notations}

We use the following conventions and notations.

- The composite of morphisms $f: X \rightarrow Y$ and $g: Y \rightarrow Z$ is usually denoted by $f g: X \rightarrow Z$. The composite of functors $F: \mathcal{C} \rightarrow \mathcal{D}$ and $G: \mathcal{D} \rightarrow \mathcal{E}$ is usually denoted by $G \circ F: \mathcal{C} \rightarrow \mathcal{E}$.

- We use the notations $\mathbb{N}=\{1,2,3, \ldots\}$ and $\mathbb{N}_{0}=\mathbb{N} \cup\{0\}$.

- Given a map $f: X \rightarrow Y$ and subsets $X^{\prime} \subseteq X, Y^{\prime} \subseteq Y$ with $X^{\prime} f \subseteq Y^{\prime}$, we write $\left.f\right|_{X^{\prime}} ^{Y^{\prime}}: X^{\prime} \rightarrow Y^{\prime}, x^{\prime} \mapsto x^{\prime} f$. Moreover, we abbreviate $\left.f\right|_{X^{\prime}}:=\left.f\right|_{X^{\prime}} ^{Y^{\prime}}$ and $\left.f\right|^{Y^{\prime}}:=$ $\left.f\right|_{X} ^{Y^{\prime}}$.

- Given integers $a, b \in \mathbb{Z}$, we write $[a, b]:=\{z \in \mathbb{Z} \mid a \leqslant z \leqslant b\}$ for the set of integers lying between $a$ and $b$. If we need to specify orientation, then we write $\lceil a, b\rceil:=(z \in \mathbb{Z} \mid a \leqslant z \leqslant b)$ for the ascending interval and $\lfloor a, b\rfloor=(z \in \mathbb{Z} \mid$ $a \geqslant z \geqslant b$ ) for the descending interval. Whereas we formally deal with tuples, we use the element notation; for example, we write $\prod_{i \in\lceil 1,3\rceil} g_{i}=g_{1} g_{2} g_{3}$ and $\prod_{i \in\lfloor 3,1\rfloor} g_{i}=g_{3} g_{2} g_{1}$ or $\left(g_{i}\right)_{i \in\lfloor 3,1\rfloor}=\left(g_{3}, g_{2}, g_{1}\right)$ for group elements $g_{1}, g_{2}, g_{3}$.

- Given tuples $\left(x_{j}\right)_{j \in A}$ and $\left(x_{j}\right)_{j \in B}$ with disjoint index sets $A$ and $B$, we write $\left(x_{j}\right)_{j \in A} \cup\left(x_{j}\right)_{j \in B}$ for their concatenation.

- Given groups $G$ and $H$, we denote by triv: $G \rightarrow H$ the trivial group homomorphism $g \mapsto 1$.

- Given a group homomorphism $\varphi: G \rightarrow H$, we denote its kernel by $\operatorname{Ker} \varphi$, its cokernel by Coker $\varphi$ and its image by $\operatorname{Im} \varphi$. Moreover, we write inc $=\operatorname{inc}{ }^{\operatorname{Ker} \varphi}$ : $\operatorname{Ker} \varphi \rightarrow G$ for the inclusion and quo $=$ quo $^{\operatorname{Coker} \varphi}: H \rightarrow \operatorname{Coker} \varphi$ for the quotient morphism.

- The distinguished point in a pointed set $X$ will be denoted by $*=*^{X}$.

- The fibre product of group homomorphisms $\varphi_{1}: G_{1} \rightarrow H$ and $\varphi_{2}: G_{2} \rightarrow H$ will be denoted by $G_{1 \varphi_{1}} \times{ }_{\varphi_{2}} G_{2}$.

\section{A remark on functoriality}

Most constructions defined below, for example M, Ch, etc., are functorial, although we only describe them on the objects of the respective source categories. For the definitions on the morphisms and other details, we refer the reader for example to $[\mathbf{2 9}]$.

\section{A remark on Grothendieck universes}

To avoid set-theoretical difficulties, we work with Grothendieck universes $[\mathbf{1}$, exp. I, sec. 0] in this article. In particular, every category has an object set and a morphism set.

We suppose given a Grothendieck universe $\mathfrak{U}$. A $\mathfrak{U}$-set is a set that is an element of $\mathfrak{U}$, and a $\mathfrak{U}$-map is a map between $\mathfrak{U}$-sets. The category of $\mathfrak{U}$-sets consisting of the set of $\mathfrak{U}$-sets, that is, of $\mathfrak{U}$, as object set and the set of $\mathfrak{U}$-maps as morphism set will be denoted by $\boldsymbol{S e t}_{(\mathfrak{U})}$. A $\mathfrak{U}$-group is a group whose underlying set is a $\mathfrak{U}$-set, and a 
$\mathfrak{U}$-group homomorphism is a group homomorphism between $\mathfrak{U}$-groups. The category of $\mathfrak{U}$-groups consisting of $\mathfrak{U}$-groups and $\mathfrak{U}$-group homomorphisms will be denoted by $\operatorname{Grp}_{(\mathfrak{U})}$.

Because we do not want to overload our text with the usage of Grothendieck universes, we may suppress them in notation, provided we work with a single fixed Grothendieck universe.

Grothendieck universes will play a role in the discussion of crossed module extensions, cf. section 2.13 .

\section{Preliminaries on simplicial objects, crossed modules, cohomology and extensions}

In this section, we recall some standard definitions and basic facts of simplicial algebraic topology and crossed modules. Concerning simplicial algebraic topology, the reader is referred for example to the books of Goerss and JARDine [16] or MAY [26], and a standard reference on crossed modules is the survey of BROWN [5].

The main purpose of this section is to fix notation and to explain how the cocycle formulas in the working base 3.1 can be deduced. The reader willing to believe the working base 3.1 can start to read at that point, occasionally looking up notation.

\subsection{Simplicial objects}

We suppose given a Grothendieck universe containing an infinite set. For $n \in \mathbb{N}_{0}$, we let $[n]$ denote the category induced by the totally ordered set $[0, n]$ with the natural order, and we let $\boldsymbol{\Delta}$ be the full subcategory in Cat defined by Ob $\boldsymbol{\Delta}:=\left\{[n] \mid n \in \mathbb{N}_{0}\right\}$. For $n \in \mathbb{N}, k \in[0, n]$, we let $\delta^{k}:[n-1] \rightarrow[n]$ be the injection that omits $k$, and for $n \in \mathbb{N}_{0}, k \in[0, n]$, we let $\sigma^{k}:[n+1] \rightarrow[n]$ be the surjection that repeats $k$.

The category of simplicial objects in a given category $\mathcal{C}$ is defined to be the functor category $\mathbf{s} \mathcal{C}:=\left(\Delta^{\mathrm{op}}, \mathcal{C}\right)$. The objects resp. morphisms of $\mathbf{s} \mathcal{C}$ are called simplicial objects in $\mathcal{C}$ resp. simplicial morphisms in $\mathcal{C}$.

Given a simplicial object $X$ in a category $\mathcal{C}$, the images of $\delta^{k}$ resp. $\sigma^{k}$ under $X$ are denoted by $\mathrm{d}_{k}=\mathrm{d}_{k}^{X}:=X_{\delta^{k}}$, called the $k$-th face, for $k \in[0, n], n \in \mathbb{N}$, resp. $\mathrm{s}_{k}=\mathrm{s}_{k}^{X}:=X_{\sigma^{k}}$, called the $k$-th degeneracy, for $k \in[0, n], n \in \mathbb{N}_{0}$. For the simplicial identities between the faces and degeneracies in our composition order, see for example [29, prop. (1.14)]. We use the ascending and descending interval notation for composites of faces resp. degeneracies, that is, we write $\mathrm{d}_{\lfloor l, k\rfloor}:=\mathrm{d}_{l} \mathrm{~d}_{l-1} \ldots \mathrm{d}_{k}$ resp. $\mathrm{s}_{\lceil k, l\rceil}:=\mathrm{s}_{k} \mathrm{~s}_{k+1} \ldots \mathrm{s}_{l}$.

Given an object $X \in \mathrm{Ob} \mathcal{C}$, we have the constant simplicial object Const $X$ in $\mathcal{C}$ with Const ${ }_{n} X:=X$ for $n \in \mathbb{N}_{0}$ and Const $_{\theta} X:=1_{X}$ for $\theta \in \Delta([m],[n]), m, n \in \mathbb{N}_{0}$.

A simplicial set resp. a simplicial map is a simplicial object resp. a simplicial morphism in $\operatorname{Set}_{(\mathfrak{U})}$ for some Grothendieck universe $\mathfrak{U}$. A simplicial group resp. a simplicial group homomorphism is a simplicial object resp. a simplicial morphism in $\operatorname{Grp}_{(\mathfrak{U})}$ for some Grothendieck universe $\mathfrak{U}$.

\subsection{The Moore complex of a simplicial group}

We suppose given a simplicial group $G$. The Moore complex of $G$ is the complex of (possibly non-abelian) groups $\mathrm{M} G$ with entries $\mathrm{M}_{n} G:=\bigcap_{k \in[1, n]} \operatorname{Ker} \mathrm{d}_{k} \leqslant G_{n}$ for $n \in$ 
$\mathbb{N}_{0}$ and differentials $\partial:=\left.\mathrm{d}_{0}\right|_{\mathrm{M}_{n} G} ^{\mathrm{M}_{n-1} G}$ for $n \in \mathbb{N}$. In particular, $\mathrm{M}_{0} G=G_{0}$. The boundary group $\mathrm{B}_{n} \mathrm{M} G$ is a normal subgroup of $G_{n}$ for all $n \in \mathbb{N}_{0}$.

\subsection{Simplicial group actions}

We suppose given a simplicial group $G$. A $G$-simplicial set consists of a simplicial set $X$ together with actions of $G_{n}$ on $X_{n}$ for $n \in \mathbb{N}_{0}$ such that $\left(g_{n} x_{n}\right) X_{\theta}=$ $\left(g_{n} G_{\theta}\right)\left(x_{n} X_{\theta}\right)$ for all $g_{n} \in G_{n}, x_{n} \in X_{n}, \theta \in \Delta([m],[n])$, where $m, n \in \mathbb{N}_{0}$. Given a $G$-simplicial set $X$, we obtain an induced simplicial structure on the sets $X_{n} / G_{n}=$ $\left\{G_{n} x_{n} \mid x_{n} \in X_{n}\right\}$ for $n \in \mathbb{N}_{0}$, and the resulting simplicial set is denoted by $X / G$.

An (abelian) $G$-simplicial module consists of a simplicial (abelian) group $M$ together with actions of $G_{n}$ on $M_{n}$ for $n \in \mathbb{N}_{0}$ such that $\left({ }^{g_{n}} m_{n}\right) M_{\theta}={ }^{g_{n} G_{\theta}}\left(m_{n} M_{\theta}\right)$ for all $\theta \in \boldsymbol{\Delta}([m],[n])$, where $m, n \in \mathbb{N}_{0}$.

\subsection{Crossed modules}

A crossed module consists of a group $G$, a (possibly non-abelian) $G$-module $M$ and a group homomorphism $\mu: M \rightarrow G$ such that $\left({ }^{g} m\right) \mu={ }^{g}(m \mu)$ and ${ }^{n \mu} m={ }^{n} m$ for all $m, n \in M, g \in G$. Here, the action of the elements of $G$ on $G$ resp. of $M$ on $M$ denote in each case the conjugation. We call $G$ the group part, $M$ the module part and $\mu$ the structure morphism of the crossed module. $\left({ }^{2}\right)$ Given a crossed module $V$ with group part $G$, module part $M$ and structure morphism $\mu$, we write $\operatorname{Gp} V:=G$, $\operatorname{Mp} V:=M$ and $\mu=\mu^{V}:=\mu$. For a list of examples of crossed modules, we refer the reader to $[\mathbf{1 4}$, sec. 2$]$.

We let $V$ and $W$ be crossed modules. A morphism of crossed modules from $V$ to $W$ consists of group homomorphisms $\varphi_{0}: \operatorname{Gp} V \rightarrow \operatorname{Gp} W$ and $\varphi_{1}: \operatorname{Mp} V \rightarrow \operatorname{Mp} W$ such that $\varphi_{1} \mu^{W}=\mu^{V} \varphi_{0}$ and such that $\left({ }^{g} m\right) \varphi_{1}={ }^{g \varphi_{0}}\left(m \varphi_{1}\right)$ holds for all $m \in \operatorname{Mp} V$, $g \in \mathrm{Gp} V$. The group homomorphisms $\varphi_{0}$ resp. $\varphi_{1}$ are said to be the group part resp. the module part of the morphism of crossed modules. Given a morphism of crossed modules $\varphi$ from $V$ to $W$ with group part $\varphi_{0}$ and module part $\varphi_{1}$, we write $\operatorname{Gp} \varphi:=\varphi_{0}$ and $\operatorname{Mp} \varphi:=\varphi_{1}$.

We let $\mathfrak{U}$ be a Grothendieck universe. A crossed module $V$ is said to be a $\mathfrak{U}$-crossed module if $\operatorname{Gp} V$ is a $\mathfrak{U}$-group and $\mathrm{Mp} V$ is a $\mathfrak{U}$ - $G$-module. The category of $\mathfrak{U}$-crossed modules consisting of $\mathfrak{U}$-crossed modules and morphisms of $\mathfrak{U}$-crossed modules will be denoted by $\mathbf{C r M o d}=\operatorname{CrMod}_{(\mathfrak{U})}$.

Notation. Given a crossed module $V$, the module part $\operatorname{Mp} V$ acts on $\operatorname{Gp} V$ by $m g:=$ $(m \mu) g$ resp. $g m:=g(m \mu)$ for $m \in \mathrm{Mp} V, g \in \mathrm{Gp} V$. Using this, we get for example ${ }^{m g} n={ }^{m}\left({ }^{g} n\right)$ and $g m=\left({ }^{g} m\right) g$ for $m, n \in \mathrm{Mp} V, g \in \mathrm{Gp} V$, cf. [31, p. 5]. Also note that $(m g) n=m(g n)$ for $m, n \in \mathrm{Mp} V, g \in \mathrm{Gp} V$.

Given a set $X$ and a map $f: \operatorname{Gp} V \rightarrow X$, we usually write $m f:=m \mu f$ for $m \in$ $\operatorname{Mp} V$. Similarly for maps $\operatorname{Gp} V \times \operatorname{Gp} V \rightarrow X$, etc.

Moreover, given crossed modules $V$ and $W$ and a morphism of crossed modules $\varphi: V \rightarrow W$, we may write $m \varphi$ and $g \varphi$ instead of $m(\operatorname{Mp} \varphi)$ and $g(\operatorname{Gp} \varphi)$. Using this, we have $(m g) \varphi=(m \varphi)(g \varphi)$ for $m \in \mathrm{Mp} V, g \in \mathrm{Gp} V$, cf. again [31, p. 5].

\footnotetext{
${ }^{2}$ In the literature, a $G$-module for a given group $G$ is often called a $G$-group while an abelian $G$ module is just a $G$-module. However, the module part of a crossed module is in general a non-abelian module over the group part; this would be more complicated to phrase using the terms from the literature.
} 


\subsection{Truncation and coskeleton}

We suppose given a simplicial group $G$. We define $\operatorname{Trunc}^{0} G:=\mathrm{M}_{0} G / \mathrm{B}_{0} \mathrm{M} G=$ $G_{0} / \mathrm{B}_{0} \mathrm{M} G$, the group segment of $G$. Moreover, we define a crossed module Trunc ${ }^{1} G$, the crossed module segment of $G$, as follows. We let $G$ Trunc ${ }^{1} G:=\mathrm{M}_{0} G=G_{0}$ and Mp Trunc ${ }^{1} G:=\mathrm{M}_{1} G / \mathrm{B}_{1} \mathrm{M} G$. Further, we let $\left(g_{1} \mathrm{~B}_{1} \mathrm{M} G\right) \mu^{\operatorname{Trunc}^{1} G}:=g_{1} \partial=g_{1} \mathrm{~d}_{0}$ for $g_{1} \in \mathrm{M}_{1} G$ and ${ }^{g_{0}}\left(g_{1} \mathrm{~B}_{1} \mathrm{M} G\right):={ }^{g_{0} \mathrm{~s}_{0}} g_{1} \mathrm{~B}_{1} \mathrm{M} G$ for $g_{i} \in \mathrm{M}_{i} G, i \in[0,1]$.

Next, we suppose given a crossed module $V$. We let $\operatorname{Trunc}_{1}^{0} V:=$ Coker $\mu$, the group segment of $V$. Moreover, we define a simplicial group $\operatorname{Cosk}_{1} V$, the coskeleton simplicial group of $V$, as follows. Denoting the elements in $(\operatorname{Mp} V)^{\times n} \times \operatorname{Gp} V$ for $n \in$ $\mathbb{N}_{0}$ by $\left(m_{i}, g\right)_{i \in\lfloor n-1,0\rfloor}:=\left(m_{i}\right)_{i \in\lfloor n-1,0\rfloor} \cup(g)$, we equip these sets with a multiplication by

$$
\left(m_{i}, g\right)_{i \in\lfloor n-1,0\rfloor}\left(m_{i}^{\prime}, g^{\prime}\right)_{i \in\lfloor n-1,0\rfloor}:=\left(m_{i}\left(\prod_{k \in\lfloor i-1,0\rfloor} m_{k}\right) g m_{i}^{\prime}, g g^{\prime}\right)_{i \in\lfloor n-1,0\rfloor}
$$

for $m_{i}, m_{i}^{\prime} \in \operatorname{Mp} V$, where $i \in\lfloor n-1,0\rfloor, g, g^{\prime} \in \mathrm{Gp} V$. The resulting group will be denoted by $\operatorname{Mp} V{ }_{n} \rtimes \operatorname{Gp} V$. For $\theta \in \boldsymbol{\Delta}([m],[n])$, we define a group homomorphism $\operatorname{Mp} V_{\theta} \rtimes \operatorname{Gp} V: \operatorname{Mp} V{ }_{n} \rtimes \operatorname{Gp} V \rightarrow \operatorname{Mp} V_{m} \rtimes \operatorname{Gp} V$ by

$$
\begin{aligned}
\left(m_{j}, g\right)_{j \in\lfloor n-1,0\rfloor}\left(\operatorname{Mp} V{ }_{\theta} \rtimes \mathrm{Gp} V\right) & :=\left(\prod_{k \in\lfloor(i+1) \theta-1, i \theta\rfloor} m_{k},\left(\prod_{k \in\lfloor 0 \theta-1,0\rfloor} m_{k}\right) g\right)_{i \in\lfloor m-1,0\rfloor} .
\end{aligned}
$$

The resulting simplicial group $\operatorname{Cosk}_{1} V:=\mathrm{Mp} V{ }_{*} \rtimes \mathrm{Gp} V$ is the coskeleton of $\left.V .{ }^{3}\right)$

Finally, we suppose given a group $G$. Then we define a $\operatorname{simplicial~group~} \operatorname{Cosk}_{0} G:=$ Const $G$, the coskeleton simplicial group of $G$. Moreover, we define a crossed module $\operatorname{Cosk}_{0}^{1} G$, the coskeleton crossed module of $G$ by $\operatorname{Gp} \operatorname{Cosk}_{0} G:=G$ and $\operatorname{Mp} \operatorname{Cosk}_{0} G:=$ $\{1\}$.

All mentioned truncation and coskeleton constructions are functorial and the resulting truncation functors are left adjoint to the resulting coskeleton functors. The unit $\varepsilon: \operatorname{id}_{\mathbf{s G r p}} \rightarrow \operatorname{Cosk}_{0} \circ \operatorname{Trunc}^{0}$ is given by $g_{n}\left(\varepsilon_{G}\right)_{n}=g_{n} \mathrm{~d}_{\lfloor n, 1\rfloor} \mathrm{B}_{0} \mathrm{M} G$ for $g_{n} \in G_{n}$, $n \in \mathbb{N}_{0}, G \in$ ObsGrp, cf. [29, prop. (4.15)]. The unit $\varepsilon: \mathrm{id}_{\mathbf{s G r p}} \rightarrow$ Cosk $_{1} \circ$ Trunc $^{1}$ fulfills $g_{0}\left(\varepsilon_{G}\right)_{0}=\left(g_{0}\right)$ for $g_{0} \in G_{0}$ and $g_{1}\left(\varepsilon_{G}\right)_{1}=\left(g_{1}\left(g_{1} \mathrm{~d}_{1} \mathrm{~s}_{0}\right)^{-1} \mathrm{~B}_{1} \mathrm{M} G, g_{1} \mathrm{~d}_{1}\right)$ for $g_{1} \in G_{1}$, $G \in$ Ob sGrp, cf. for example [29, def. (6.11), def. (6.15), rem. (6.14), prop. (6.9), th. (5.25)].

We have $\operatorname{Trunc}^{0} \circ \operatorname{Cosk}_{0} \cong \mathrm{id}_{\text {Grp }}$, Trunc ${ }^{1} \circ \operatorname{Cosk}_{1} \cong \mathrm{id}_{\text {CrMod }}$ and $\operatorname{Trunc}_{1}^{0} \circ \operatorname{Cosk}_{0}^{1}$ $\cong \operatorname{id}_{\text {Grp }}$, as well as $\operatorname{Cosk}_{0}=\operatorname{Cosk}_{1} \circ \operatorname{Cosk}_{0}^{1}$ and $\operatorname{Trunc}^{0}=\operatorname{Trunc}_{1}^{0} \circ \operatorname{Trunc}^{1}$.

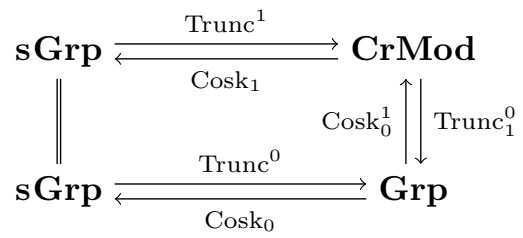

\footnotetext{
${ }^{3}$ The category of crossed modules is equivalent to the category of (strict) categorical groups, cf. [6, thm. 1]. The coskeleton functor from crossed modules to simplicial groups can be obtained via a nerve functor from the category of categorical groups to the category of simplicial groups. Cf. [7, sec. 1] and [29, ch. VI, $\S \S 1-2]$. For another truncation-coskeleton-pair, cf. [2, exp. V, sec. 7.1] and [11, sec. $(0.7)]$.
} 
Given a group $G$, we have $\mathrm{M}\left(\operatorname{Cosk}_{0} G\right)=(\ldots \longrightarrow 1 \longrightarrow 1 \longrightarrow G)$, and given a crossed module $V$, we have $\mathrm{M}\left(\operatorname{Cosk}_{1} V\right)=\left(\ldots \longrightarrow 1 \longrightarrow \mathrm{Mp} V \stackrel{\mu^{V}}{\longrightarrow} \mathrm{Gp} V\right)$; cf. $[\mathbf{2 9}$, prop. (6.22)].

\subsection{Homotopy groups}

For a simplicial group $G$, we call $\pi_{n}(G):=\mathrm{H}_{n} \mathrm{M} G$ the $n$-th homotopy group of $G$ for $n \in \mathbb{N}_{0}$. It is abelian for $n \in \mathbb{N}$, and we have $\pi_{0}=$ Trunc $^{0}$.

The homotopy groups of a crossed module $V$ are defined by $\pi_{0}(V):=$ Coker $\mu=$ $\operatorname{Gp} V / \operatorname{Im} \mu, \pi_{1}(V):=\operatorname{Ker} \mu$ and $\pi_{n}(V):=\{1\}$ for $n \in \mathbb{N}_{0} \backslash\{0,1\}$. The first homotopy group $\pi_{1}(V)$ carries the structure of an abelian $\pi_{0}(V)$-module [5, sec. 3.1, sec. 3.2], where the action of $\pi_{0}(V)$ on $\pi_{1}(V)$ is induced by the action of $\operatorname{Gp} V$ on $\mathrm{Mp} V$, that is, for $k \in \pi_{1}(V)$ and $p \in \pi_{0}(V)$ we have ${ }^{p} k={ }^{g} k$ for any $g \in \operatorname{Gp} V$ with $g(\operatorname{Im} \mu)=p$.

For a crossed module $V$, we have $\pi_{n}(V) \cong \pi_{n}\left(\operatorname{Cosk}_{1} V\right)$ for all $n \in \mathbb{N}_{0}$, cf. for example [29, ch. VI, §3]. Moreover, given a simplicial group $G$, we have $\pi_{n}(G)=$ $\pi_{n}\left(\operatorname{Trunc}^{1} G\right)$ for $n \in\{0,1\} .\left({ }^{4}\right)$

\subsection{Semidirect product decomposition}

We suppose given a simplicial group $G$. The group of $n$-simplices $G_{n}$, where $n \in \mathbb{N}_{0}$, is isomorphic to an iterated semidirect product in terms of the entries $\mathrm{M}_{k} G$ for $k \in[0, n]$ of the Moore complex $\mathrm{M} G$. For example, we have $G_{0}=\mathrm{M}_{0} G$ and $G_{1} \cong$ $\mathrm{M}_{1} G \rtimes \mathrm{M}_{0} G$ and $G_{2} \cong\left(\mathrm{M}_{2} G \rtimes \mathrm{M}_{1} G\right) \rtimes\left(\mathrm{M}_{1} G \rtimes \mathrm{M}_{0} G\right)$, where $\mathrm{M}_{0} G$ acts on $\mathrm{M}_{1} G$ via ${ }^{g_{0}} g_{1}:={ }^{g_{0} \mathrm{~s}_{0}} g_{1}$ for $g_{i} \in \mathrm{M}_{i} G, i \in\{0,1\}, \mathrm{M}_{1} G$ acts on $\mathrm{M}_{2} G$ via ${ }^{g_{1}} g_{2}:={ }^{g_{1} \mathrm{~s}_{0}} g_{2}$ for $g_{i} \in$ $\mathrm{M}_{i} G, i \in\{1,2\}$ and $\mathrm{M}_{1} G \rtimes \mathrm{M}_{0} G$ acts on $\mathrm{M}_{2} G \rtimes \mathrm{M}_{1} G$ via

$$
{ }^{\left(g_{1}, g_{0}\right)}\left(g_{2}, h_{1}\right):=\left({ }^{\left(g_{1} \mathrm{~s}_{1}\right)\left(g_{0} \mathrm{~s}_{0} \mathrm{~s}_{1}\right)}\left(g_{2}\left(h_{1} \mathrm{~s}_{0}\right)\right)^{\left(g_{1} \mathrm{~s}_{0}\right)\left(g_{0} \mathrm{~s}_{0} \mathrm{~s}_{1}\right)}\left(\left(h_{1} \mathrm{~s}_{0}\right)^{-1}\right),{ }^{g_{1}\left(g_{0} \mathrm{~s}_{0}\right)} h_{1}\right)
$$

for $g_{i}, h_{i} \in \mathrm{M}_{i} G, i \in[0,2]$. The isomorphisms are given by

$$
\begin{aligned}
& \varphi_{1}: G_{1} \rightarrow \mathrm{M}_{1} G \rtimes \mathrm{M}_{0} G, g_{1} \mapsto\left(g_{1}\left(g_{1} \mathrm{~d}_{1} \mathrm{~s}_{0}\right)^{-1}, g_{1} \mathrm{~d}_{1}\right), \\
& \varphi_{1}^{-1}: \mathrm{M}_{1} G \rtimes \mathrm{M}_{0} G \rightarrow G_{1},\left(g_{1}, g_{0}\right) \mapsto g_{1}\left(g_{0} \mathrm{~s}_{0}\right)
\end{aligned}
$$

and

$$
\begin{aligned}
& \varphi_{2}: G_{2} \rightarrow\left(\mathrm{M}_{2} G \rtimes \mathrm{M}_{1} G\right) \rtimes\left(\mathrm{M}_{1} G \rtimes \mathrm{M}_{0} G\right), \\
& g_{2} \mapsto\left(\left(g_{2}\left(g_{2} \mathrm{~d}_{2} \mathrm{~s}_{1}\right)^{-1}\left(g_{2} \mathrm{~d}_{2} \mathrm{~s}_{0}\right)\left(g_{2} \mathrm{~d}_{1} \mathrm{~s}_{0}\right)^{-1},\left(g_{2} \mathrm{~d}_{1}\right)\left(g_{2} \mathrm{~d}_{2}\right)^{-1}\right),\right. \\
&\left.\quad\left(\left(g_{2} \mathrm{~d}_{2}\right)\left(g_{2} \mathrm{~d}_{2} \mathrm{~d}_{1} \mathrm{~s}_{0}\right)^{-1}, g_{2} \mathrm{~d}_{2} \mathrm{~d}_{1}\right)\right), \\
& \varphi_{2}^{-1}:\left(\mathrm{M}_{2} G \rtimes \mathrm{M}_{1} G\right) \rtimes\left(\mathrm{M}_{1} G \rtimes \mathrm{M}_{0} G\right) \rightarrow G_{2}, \\
&\left(\left(g_{2}, h_{1}\right),\left(g_{1}, g_{0}\right)\right) \mapsto g_{2}\left(h_{1} \mathrm{~s}_{0}\right)\left(g_{1} \mathrm{~s}_{1}\right)\left(g_{0} \mathrm{~s}_{0} \mathrm{~s}_{1}\right) .
\end{aligned}
$$

For more details, see $[\mathbf{9}]$ or $[\mathbf{2 9}$, ch. IV, $\S 2]$.

\subsection{Cohomology of simplicial sets}

We suppose given a simplicial set $X$ and an abelian group $A$. The cochain complex of $X$ with coefficients in $A$ is the complex of abelian groups $\operatorname{Ch}_{\text {sSet }}(X, A)$ with abelian groups $\mathrm{Ch}_{\mathrm{sSet}}^{n}(X, A):=\operatorname{Map}\left(X_{n}, A\right)$ for $n \in \mathbb{N}_{0}$ and differentials defined by $x(c \partial):=$

\footnotetext{
${ }^{4}$ In particular, given a simplicial group $G$, we have $\pi_{n}(G) \cong \pi_{n}\left(\operatorname{Cosk}_{1} \operatorname{Trunc}^{1} G\right)$ for $n \in\{0,1\}$.
} This property fails for the truncation-coskeleton pair in [11, sec. $(0.7)]$. 
$\sum_{k \in[0, n+1]}(-1)^{k}\left(x \mathrm{~d}_{k}\right) c$ for $x \in X_{n+1}, c \in \mathrm{Ch}_{\mathbf{s S e t}}^{n}(X, A)$. We call $\mathrm{Ch}_{\mathbf{s S e t}}^{n}(X, A)$ the $n$-th cochain group of $X$ with coefficients in $A$. Moreover, we define the $n$-th cocycle group $\mathrm{Z}_{\mathrm{sSet}}^{n}(X, A):=\mathrm{Z}^{n} \mathrm{Ch}_{\mathrm{sSet}}(X, A)$, the $n$-th coboundary group $\mathrm{B}_{\mathrm{sSet}}^{n}(X, A):=$ $\mathrm{B}^{n} \mathrm{Ch}_{\mathrm{sSet}}(X, A)$ and the $n$-th cohomology group $\mathrm{H}_{\mathrm{sSet}}^{n}(X, A):=\mathrm{H}^{n} \mathrm{Ch}_{\mathrm{sSet}}(X, A)=$ $\mathrm{Z}_{\mathrm{sSet}}^{n}(X, A) / \mathrm{B}_{\mathrm{sSet}}^{n}(X, A)$ of $X$ with coefficients in $A\left({ }^{5}\right)$. An element $c \in \mathrm{Ch}_{\mathrm{sSet}}^{n}(X, A)$ resp. $z \in \mathrm{Z}_{\mathrm{sSet}}^{n}(X, A)$ resp. $b \in \mathrm{B}_{\mathrm{sSet}}^{n}(X, A)$ resp. $h \in \mathrm{H}_{\mathrm{sSet}}^{n}(X, A)$ is said to be an $n$ cochain resp. an $n$-cocycle resp. an $n$-coboundary resp. an $n$-cohomology class of $X$ with coefficients in $A$.

\subsection{Cohomology of simplicial groups with coefficients in an abelian group}

Cohomology of simplicial sets can be used to define cohomology of a simplicial group $G$. This is done via the Kan classifying simplicial set $\overline{\mathrm{W}} G$ of $G$, see KAN [21, def. 10.3], which is given by $\overline{\mathrm{W}}_{n} G:=\times_{j \in\lfloor n-1,0\rfloor} G_{j}$ for all $n \in \mathbb{N}_{0}$ and

$$
\left(g_{j}\right)_{j \in\lfloor n-1,0\rfloor}\left(\overline{\mathrm{W}}_{\theta} G\right):=\left(\prod_{j \in\lfloor(i+1) \theta-1, i \theta\rfloor} g_{j} G_{\left.\theta\right|_{[i]} ^{[j]}}\right)_{i \in\lfloor m-1,0\rfloor}
$$

for $\left(g_{j}\right)_{j \in\lfloor n-1,0\rfloor} \in \overline{\mathrm{W}}_{n} G$ and $\theta \in \boldsymbol{\Delta}_{\boldsymbol{\Delta}}([m],[n])$, where $m, n \in \mathbb{N}_{0}$, cf. for example [29, rem. (4.19)]. In particular, the faces are given by

$$
\left(g_{j}\right)_{j \in\lfloor n-1,0\rfloor} \mathrm{d}_{k}^{\overline{\mathrm{W}} G}= \begin{cases}\left(g_{j+1} \mathrm{~d}_{0}^{G}\right)_{j \in\lfloor n-2,0\rfloor} & \text { for } k=0, \\ \left(g_{j+1} \mathrm{~d}_{k}^{G}\right)_{j \in\lfloor n-2, k\rfloor} \cup\left(\left(g_{k} \mathrm{~d}_{k}^{G}\right) g_{k-1}\right) & \\ \cup\left(g_{j}\right)_{j \in\lfloor k-2,0\rfloor} & \text { for } k \in[1, n-1], \\ \left(g_{j}\right)_{j \in\lfloor n-2,0\rfloor} & \text { for } k=n,\end{cases}
$$

for $\left(g_{j}\right)_{j \in\lfloor n-1,0\rfloor} \in \overline{\mathrm{W}}_{n} G, n \in \mathbb{N}$. The cochain complex of $G$ with coefficients in an abelian group $A$ is defined to be $\mathrm{Ch}(G, A)=\mathrm{Ch}_{\mathbf{s G r p}}(G, A):=\mathrm{Ch}_{\mathrm{sSet}}(\overline{\mathrm{W}} G, A)$. Moreover, we define the $n$-th cocycle group $\mathrm{Z}^{n}(G, A)=\mathrm{Z}_{\mathrm{sGrp}}^{n}(G, A):=\mathrm{Z}_{\mathrm{sSet}}^{n}(\overline{\mathrm{W}} G, A)$, etc., for $n \in \mathbb{N}_{0}$. The differentials of $\operatorname{Ch}(G, A)$ are given by

$$
\begin{aligned}
& \left(g_{j}\right)_{j \in\lfloor n, 0\rfloor}(c \partial)=\left(g_{j+1} \mathrm{~d}_{0}\right)_{j \in\lfloor n-1,0\rfloor} c \\
& \quad+\sum_{k \in[1, n]}(-1)^{k}\left(\left(g_{j+1} \mathrm{~d}_{k}\right)_{j \in\lfloor n-1, k\rfloor} \cup\left(\left(g_{k} \mathrm{~d}_{k}\right) g_{k-1}\right) \cup\left(g_{j}\right)_{j \in\lfloor k-2,0\rfloor}\right) c \\
& \quad+(-1)^{n+1}\left(g_{j}\right)_{j \in\lfloor n-1,0\rfloor} c
\end{aligned}
$$

for $\left(g_{j}\right)_{j \in\lfloor n, 0\rfloor} \in \overline{\mathrm{W}}_{n+1} G, c \in \mathrm{Ch}^{n}(G, A), n \in \mathbb{N}_{0}$.

Instead of $\overline{\mathrm{W}} G$, one can also use Diag $\mathrm{N} G$, the diagonal simplicial set of the nerve of $G$, see for example [15, app. Q.3], [19, p. 41] and [29]. The simplicial sets Diag NG and $\overline{\mathrm{W}} G$ are simplicially homotopy equivalent [30, thm.], cf. also [10, thm. 1.1], and thus $\mathrm{H}^{n}(G, A)=\mathrm{H}_{\mathrm{sSet}}^{n}(\overline{\mathrm{W}} G, A) \cong \mathrm{H}_{\text {sSet }}^{n}(\operatorname{Diag} \mathrm{N} G, A)$ for $n \in \mathbb{N}_{0}$, where $A$ is an abelian group.

\footnotetext{
${ }^{5}$ In the literature, $\mathrm{Z}_{\mathrm{s} \text { Set }}^{n}(X, A)$ resp. $\mathrm{B}_{\mathrm{sSet}}^{n}(X, A)$ resp. $\mathrm{H}_{\mathrm{s} S e t}^{n}(X, A)$ are often defined by an isomorphic complex of abelian groups (cf. for example [29, def. (2.18)]) and are just denoted $\mathrm{Z}^{n}(X, A)$ resp. $\mathrm{B}^{n}(X, A)$ resp. $\mathrm{H}^{n}(X, A)$.
} 


\subsection{Cohomology of simplicial groups with coefficients in an abelian module}

To generalise cohomology of a simplicial group $G$ with coefficients in an abelian group $A$ to cohomology with coefficients in an abelian $\pi_{0}(G)$-module $M$, we have to introduce a further notion on simplicial sets: There is a shift functor Sh: $\boldsymbol{\Delta} \rightarrow \boldsymbol{\Delta}$ given by $\operatorname{Sh}[n]:=[n+1]$ as well as $i(\operatorname{Sh} \theta):=i \theta$ for $i \in[0, m]$ and $(m+1)(\operatorname{Sh} \theta):=n+1$, for $\theta \in \boldsymbol{\Delta}([m],[n]), m, n \in \mathbb{N}_{0}$. Given a simplicial set $X$, the path simplicial set of $X$ is the simplicial set $\mathrm{P} X:=X \circ(\mathrm{Sh})^{\mathrm{op}}$, which is simplicially homotopy equivalent to Const $X_{0}[32,8.3 .14]$. The faces $\mathrm{d}_{n+1}^{X}: \mathrm{P}_{n} X \rightarrow X_{n}$ for $n \in \mathbb{N}_{0}$ form a simplicial map $\mathrm{P} X \rightarrow X$.

Now we follow QuILLEN [27, ch. II, p. 6.16] and consider for a given simplicial group $G$ the Kan resolving simplicial set $\mathrm{W} G:=\mathrm{PW} G$. The simplicial group $G$ acts on W $G$ by $g\left(g_{j}\right)_{j \in\lfloor n, 0\rfloor}:=\left(g g_{n}\right) \cup\left(g_{j}\right)_{j \in\lfloor n-1,0\rfloor}$ for $g \in G_{n},\left(g_{j}\right)_{j \in\lfloor n, 0\rfloor} \in \mathrm{W}_{n} G, n \in \mathbb{N}_{0}$, and the simplicial map $\mathrm{W} G \rightarrow \overline{\mathrm{W}} G$ given by $\mathrm{W}_{n} G \rightarrow \overline{\mathrm{W}}_{n} G,\left(g_{j}\right)_{j \in\lfloor n, 0\rfloor} \mapsto\left(g_{j}\right)_{j \in\lfloor n-1,0\rfloor}$ induces a simplicial bijection $\mathrm{W} G / G \rightarrow \overline{\mathrm{W}} G$.

We suppose given an abelian $\pi_{0}(G)$-module $M$. Then Const $M$ is a simplicial abelian $\pi_{0}(G)$-module, and the unit $\varepsilon: \operatorname{id}_{\mathbf{s G r p}} \rightarrow \operatorname{Cosk}_{0} \circ \pi_{0}$ of the adjunction $\pi_{0}=$ Trunc $^{0} \dashv$ Cosk $_{0}$ turns Const $M$ into an abelian $G$-simplicial module via $g_{n} x_{n}:=$ $\left(g_{n}\left(\varepsilon_{G}\right)_{n}\right) x_{n}=\left(g_{n} \mathrm{~d}_{\lfloor n, 1\rfloor} \mathrm{B}_{0} \mathrm{M} G\right) x_{n}$ for $g_{n} \in G_{n}, x_{n} \in M_{n}, n \in \mathbb{N}_{0}$. Since $\varepsilon_{G}$ is a simplicial group homomorphism, we have $g_{n} G_{\theta}\left(\varepsilon_{G}\right)_{m}=g_{n}\left(\varepsilon_{G}\right)_{n}$ for all $g_{n} \in G_{n}, \theta \in$ $\Delta([m],[n]), m, n \in \mathbb{N}_{0}$.

We consider the subcomplex $\mathrm{Ch}_{\text {hom }}(G, M)=\mathrm{Ch}_{\mathbf{s G r p}, \text { hom }}(G, M)$ of the cochain complex $\mathrm{Ch}_{\mathrm{sSet}}(\mathrm{W} G, M)$ with entries $\mathrm{Ch}_{\text {hom }}^{n}(G, M):=\operatorname{Map}_{G_{n}}\left(\mathrm{~W}_{n} G, M\right)$ and differentials given by

$$
\left(g_{j}\right)_{j \in\lfloor n+1,0\rfloor}(c \partial):=\sum_{k \in[0, n+1]}(-1)^{k}\left(\left(g_{j}\right)_{j \in\lfloor n+1,0\rfloor} \mathrm{d}_{k}\right) c
$$

for $\left(g_{j}\right)_{j \in\lfloor n+1,0\rfloor} \in \mathrm{W}_{n+1} G, c \in \mathrm{Ch}_{\text {hom }}^{n}(G, M), n \in \mathbb{N}_{0}$, called the homogeneous cochain complex of $G$ with coefficients in $M$. We want to introduce an isomorphic variant of $\mathrm{Ch}_{\text {hom }}(G, M)$ using transport of structure. We have

$$
\begin{aligned}
& \left(g_{j}\right)_{j \in\lfloor n+1,0\rfloor}(c \partial)=g_{n+1} \mathrm{~d}_{\lfloor n+1,1\rfloor} \mathrm{B}_{0} \mathrm{M} G \cdot\left(\left((1) \cup\left(g_{j+1} \mathrm{~d}_{0}\right)_{j \in\lfloor n-1,0\rfloor}\right) c\right. \\
& \quad+\sum_{k \in[1, n]}(-1)^{k}\left((1) \cup\left(g_{j+1} \mathrm{~d}_{k}\right)_{j \in\lfloor n-1, k\rfloor} \cup\left(\left(g_{k} \mathrm{~d}_{k}\right) g_{k-1}\right) \cup\left(g_{j}\right)_{j \in\lfloor k-2,0\rfloor}\right) c \\
& \left.\quad+(-1)^{n+1}\left(g_{n} \mathrm{~d}_{\lfloor n, 1\rfloor} \mathrm{B}_{0} \mathrm{M} G\right) \cdot\left((1) \cup\left(g_{j}\right)_{j \in\lfloor n-1,0\rfloor}\right) c\right)
\end{aligned}
$$

for $\left(g_{j}\right)_{j \in\lfloor n+1,0\rfloor} \in \mathrm{W}_{n+1} G, c \in \mathrm{Ch}_{\text {hom }}^{n}(G, M), n \in \mathbb{N}_{0}$. Thus $\mathrm{Ch}_{\text {hom }}(G, M)$ is isomorphic to a complex $\operatorname{Ch}(G, M)$, called the cochain complex of $G$ with coefficients in the abelian $\pi_{0}(G)$-module $M$, with entries $\operatorname{Ch}^{n}(G, M):=\operatorname{Map}\left(\times_{j \in\lfloor n-1,0\rfloor} G_{j}, M\right)=$ $\mathrm{Ch}_{\mathrm{sSet}}^{n}(\overline{\mathrm{W}} G, M)$ and differentials given by

$$
\begin{aligned}
& \left(g_{j}\right)_{j \in\lfloor n, 0\rfloor}(c \partial)=\left(g_{j+1} \mathrm{~d}_{0}\right)_{j \in\lfloor n-1,0\rfloor} c \\
& \quad+\sum_{k \in[1, n]}(-1)^{k}\left(\left(g_{j+1} \mathrm{~d}_{k}\right)_{j \in\lfloor n-1, k\rfloor} \cup\left(\left(g_{k} \mathrm{~d}_{k}\right) g_{k-1}\right) \cup\left(g_{j}\right)_{j \in\lfloor k-2,0\rfloor}\right) c \\
& \quad+(-1)^{n+1}\left(g_{n} \mathrm{~d}_{\lfloor n, 1\rfloor} \mathrm{B}_{0} \mathrm{M} G\right) \cdot\left(g_{j}\right)_{j \in\lfloor n-1,0\rfloor} c
\end{aligned}
$$


for $\left(g_{j}\right)_{j \in\lfloor n, 0\rfloor} \in \overline{\mathrm{W}}_{n+1} G, \quad c \in \mathrm{Ch}^{n}(G, M), \quad n \in \mathbb{N}_{0}$, and where an isomorphism $\varphi: \mathrm{Ch}_{\text {hom }}(G, M) \rightarrow \operatorname{Ch}(G, M)$ is given by $\left(g_{j}\right)_{j \in\lfloor n-1,0\rfloor}\left(c \varphi^{n}\right)=\left((1) \cup\left(g_{j}\right)_{j \in\lfloor n-1,0\rfloor}\right) c$ for $\left(g_{j}\right)_{j \in\lfloor n-1,0\rfloor} \in \overline{\mathrm{W}}_{n} G, c \in \mathrm{Ch}_{\text {hom }}^{n}(G, M), n \in \mathbb{N}_{0}$. Moreover, we set $\mathrm{Z}_{\mathbf{s G r p}}^{n}(G, M)=$ $\mathrm{Z}^{n}(G, M):=\mathrm{Z}^{n}(\mathrm{Ch}(G, M))$, etc., and call $\mathrm{Ch}^{n}(G, M)$ the $n$-th cochain group of $G$ with coefficients in $M$, etc. We see that this definition coincides with $\operatorname{Ch}(G, A)$ for an abelian group $A$ considered as an abelian $\pi_{0}(G)$-module with the trivial action of $\pi_{0}(G)$.

Isomorphic substitution of $G$ with its semidirect product decomposition, cf. section 2.7, leads to an isomorphic substitution of the cochain $\operatorname{complex} \mathrm{Ch}(G, M)$ to the analysed cochain complex $\mathrm{Ch}_{\text {an }}(G, M)=\mathrm{Ch}_{\mathbf{s G r p}, \text { an }}(G, M)$. Similarly, isomorphic substitution yields $\mathrm{Z}_{\text {an }}^{n}(G, M)=\mathrm{Z}_{\mathbf{s G r p}, \text { an }}^{n}(G, M)$, etc., and we call $\mathrm{Ch}_{\text {an }}^{n}(G, M)$ the $n$-th analysed cochain group of $G$ with coefficients in $M$, etc. See 3.1 for formulas in low dimensions.

Altogether, we have $\mathrm{Ch}_{\text {hom }}(G, M) \cong \mathrm{Ch}(G, M) \cong \mathrm{Ch}_{\text {an }}(G, M)$.

\subsection{Cohomology of groups and cohomology of crossed modules}

Since groups and crossed modules can be considered as truncated simplicial groups, the cohomology groups of these algebraic objects is defined via cohomology of simplicial groups.

Given a group $G$ and an abelian $G$-module $M$, we define the cochain complex $\mathrm{Ch}(G, M)=\mathrm{Ch}_{\mathbf{G r p}}(G, M):=\mathrm{Ch}_{\mathbf{s G r p}}\left(\operatorname{Cosk}_{0} G, M\right)$ of $G$ with coefficients in $M$. Similarly, we set $\mathrm{Z}^{n}(G, M)=\mathrm{Z}_{\mathbf{G r p}}^{n}(G, M):=\mathrm{Z}_{\mathbf{s G r p}}^{n}\left(\operatorname{Cosk}_{0} G, M\right)$ for $n \in \mathbb{N}_{0}$, etc., and call $\mathrm{Ch}^{n}(G, M)$ the $n$-th cochain group of $G$ with coefficients in $M$, etc. Since $\overline{\mathrm{W}}$ Cosk $_{0} G=$ $\mathrm{NG}$, where $\mathrm{N}$ is the nerve functor for groups, this definition of cohomology coincides with the standard one via $\mathrm{B} G:=\mathrm{N} G$ and $\mathrm{E} G:=\mathrm{PB} G$.

Given a crossed module $V$ and an abelian $\pi_{0}(V)$-module $M$, we define the cochain complex $\mathrm{Ch}(V, M)=\mathrm{Ch}_{\mathbf{C r M o d}}(V, M):=\mathrm{Ch}_{\mathbf{s G r p}}\left(\operatorname{Cosk}_{1} V, M\right)$ of $V$ with coefficients in $M$. Similarly, we set $\mathrm{Z}^{n}(V, M)=\mathrm{Z}_{\mathbf{C r M o d}}^{n}(V, M):=\mathrm{Z}_{\mathbf{s G r p}}^{n}\left(\operatorname{Cosk}_{1} V, M\right)$ for $n \in \mathbb{N}_{0}$, etc., and call $\mathrm{Ch}^{n}(V, M)$ the $n$-th cochain group of $V$ with coefficients in $M$, etc.

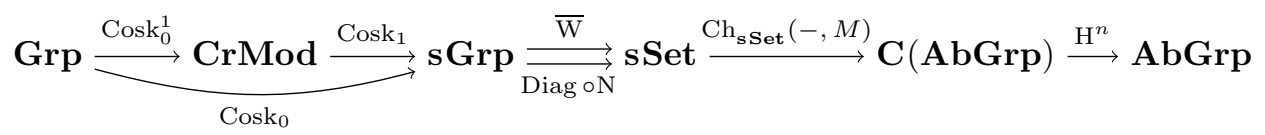

The semidirect product decomposition of $\operatorname{Cosk}_{1} V$ is - up to simplified notation - already built into the definition of $\operatorname{Cosk}_{1} V$. So the cochain complex and the analysed cochain complex of $\operatorname{Cosk}_{1} V$ are essentially equal. Therefore there is no need to explicitly introduce analysed cochains for crossed modules.

ELLIS defined in $[\mathbf{1 4}$, sec. 3] the cohomology of a crossed module $V$ with coefficients in an abelian group $A$ via the composition Diag oN, where $\mathrm{N}$ denotes the nerve functor for simplicial groups and Diag denotes the diagonal simplicial set functor for bisimplicial sets. In this article, we will make use of the Kan classifying simplicial set functor $\overline{\mathrm{W}}$ instead of Diag $\circ \mathrm{N}$ since $\overline{\mathrm{W}}$ provides smaller objects, which is more convenient for direct calculations. For example, a 2-cocycle in $\mathrm{Z}^{2}\left(\operatorname{Diag~N~Cosk}_{1} V, A\right)$ is a map $(\operatorname{Mp} V)^{\times 4} \times(\operatorname{Gp} V)^{\times 2} \rightarrow A$, while a 2-cocycle in $\mathrm{Z}^{2}(V, A)=\mathrm{Z}^{2}\left(\overline{\mathrm{W}} \operatorname{Cosk}_{1} V, A\right)$ is a map $\operatorname{Mp} V \times(\operatorname{Gp} V)^{\times 2} \rightarrow A$. 


\subsection{Pointed cochains}

We let $G$ be a simplicial group and $M$ be an abelian $\pi_{0}(G)$-module. As we have seen above, an $n$-cochain of $G$ with coefficients in $M$ is just a map $c: \overline{\mathrm{W}}_{n} G \rightarrow M$, where $n \in$ $\mathbb{N}_{0}$. Since the sets $\overline{\mathrm{W}}_{n} G=\times_{j \in\lfloor n-1,0\rfloor} G_{j}$ carry structures as direct products of groups for $n \in \mathbb{N}_{0}$, they are pointed in a natural way with $1=(1)_{\lfloor n-1,0\rfloor}$ as distinguished points. Moreover, the module $M$ is in particular an abelian group and therefore pointed with 0 as distinguished point. An $n$-cochain $c \in \mathrm{Ch}^{n}(G, M)$ is said to be pointed if it is a pointed map, that is, if $1 c=0$. The subset of $\mathrm{Ch}^{n}(G, M)$ consisting of all pointed $n$-cochains of $G$ with coefficients in $M$ will be denoted by $\mathrm{Ch}_{\mathrm{pt}}^{n}(G, M):=$ $\left\{c \in \mathrm{Ch}^{n}(G, M) \mid c\right.$ is pointed $\}$. We set $\mathrm{Z}_{\mathrm{pt}}^{n}(G, M):=\mathrm{Ch}_{\mathrm{pt}}^{n}(G, M) \cap \mathrm{Z}^{n}(G, M)$ for the set of pointed $n$-cocycles, $\mathrm{B}_{\mathrm{pt}}^{n}(G, M):=\mathrm{Ch}_{\mathrm{pt}}^{n}(G, M) \cap \mathrm{B}^{n}(G, M)$ for the set of pointed $n$-coboundaries and $\mathrm{H}_{\mathrm{pt}}^{n}(G, M):=\mathrm{Z}_{\mathrm{pt}}^{n}(G, M) / \mathrm{B}_{\mathrm{pt}}^{n}(G, M)$ for the set of pointed $n$ cohomology classes of $G$ with coefficients in $M$.

We suppose given an odd natural number $n \in \mathbb{N}$. Every $n$-cocycle $z \in \mathrm{Z}^{n}(G, M)$ is pointed, and hence we have $\mathrm{Z}_{\mathrm{pt}}^{n}(G, M)=\mathrm{Z}^{n}(G, M), \mathrm{B}_{\mathrm{pt}}^{n}(G, M)=\mathrm{B}^{n}(G, M)$ and $\mathrm{H}_{\mathrm{pt}}^{n}(G, M)=\mathrm{H}^{n}(G, M)$. Moreover, we have $\mathrm{B}_{\mathrm{pt}}^{n+1}(G, M)=\left(\mathrm{Ch}_{\mathrm{pt}}^{n}(G, M)\right) \partial$.

So we suppose given an even natural number $n \in \mathbb{N}$ and an $n$-cocycle $z \in \mathrm{Z}^{n}(G, M)$. The pointisation of $z$ is given by $z^{\mathrm{pt}}:=z-\mathrm{p}_{z} \partial$, where the pointiser of $z$ is defined to be the $(n-1)$-cochain $\mathrm{p}_{z} \in \mathrm{Ch}^{n-1}(G, M)$ given by $\left(g_{j}\right)_{j \in\lfloor n-2,0\rfloor} \mathrm{p}_{z}:=(1)_{j \in\lfloor n-1,0\rfloor} z$ for $g_{j} \in G_{j}, j \in\lfloor n-2,0\rfloor$. We obtain

$$
\left(g_{j}\right)_{j \in\lfloor n-1,0\rfloor} z^{\mathrm{pt}}=\left(g_{j}\right)_{j \in\lfloor n-1,0\rfloor} z-g_{n-1} \mathrm{~d}_{\lfloor n-1,1\rfloor} \mathrm{B}_{0} \mathrm{M} G \cdot(1)_{j \in\lfloor n-1,0\rfloor} z
$$

for $g_{j} \in G_{j}, j \in\lfloor n-1,0\rfloor$. Thus the pointisation $z^{\mathrm{pt}}$ of every $z \in \mathrm{Z}^{n}(G, M)$ is pointed. We have $\mathrm{Z}_{\mathrm{pt}}^{n}(G, M)=\left\{z \in \mathrm{Z}^{n}(G, M) \mid z^{\mathrm{pt}}=z\right\}$ and the embedding $\mathrm{Z}_{\mathrm{pt}}^{n}(G, M) \rightarrow$ $\mathrm{Z}^{n}(G, M)$ and the pointisation homomorphism $\mathrm{Z}^{n}(G, M) \rightarrow \mathrm{Z}_{\mathrm{pt}}^{n}(G, M), z \mapsto z^{\mathrm{pt}}$ induce mutually inverse isomorphisms between $\mathrm{H}_{\mathrm{pt}}^{n}(G, M)$ and $\mathrm{H}^{n}(G, M)$.

Altogether, we have $\mathrm{H}^{n}(G, M) \cong \mathrm{H}_{\mathrm{pt}}^{n}(G, M)$ for all $n \in \mathbb{N}$.

Given a crossed module $V$ and an abelian $\pi_{0}(V)$-module $M$, we write $\mathrm{Ch}_{\mathrm{pt}}(V, M)$ $:=\mathrm{Ch}_{\mathrm{pt}}\left(\operatorname{Cosk}_{1} V, M\right)$, etc. Similarly, given a group $G$ and an abelian $G$-module $M$, we write $\mathrm{Ch}_{\mathrm{pt}}(G, M):=\mathrm{Ch}_{\mathrm{pt}}\left(\operatorname{Cosk}_{0} G, M\right)$, etc.

\subsection{Crossed module extensions}

We suppose given a group $\Pi_{0}$ and an abelian $\Pi_{0}$-module $\Pi_{1}$, which will be written multiplicatively.

A crossed module extension of $\Pi_{0}$ with $\Pi_{1}$ consists of a crossed module $E$ together with a group monomorphism $\iota: \Pi_{1} \rightarrow \mathrm{Mp} E$ and a group epimorphism $\pi:$ Gp $E \rightarrow \Pi_{0}$ such that

$$
\Pi_{1} \stackrel{\iota}{\longrightarrow} \mathrm{Mp} E \stackrel{\mu}{\longrightarrow} \mathrm{Gp} E \stackrel{\pi}{\longrightarrow} \Pi_{0}
$$

is an exact sequence of groups and such that the induced action of $\Pi_{0}$ on $\Pi_{1}$ caused by the action of the crossed module $E$ coincides with the a priori given action of $\Pi_{0}$ on $\Pi_{1}$, that is, such that ${ }^{g}(k \iota)=\left({ }^{g \pi} k\right) \iota$ for $g \in \mathrm{Gp} E$ and $k \in \Pi_{1}$. The group homomorphisms $\iota$ resp. $\pi$ are said to be the canonical monomorphism resp. the canonical epimorphism of the crossed module extension $E$. Given a crossed module extension $E$ of $\Pi_{0}$ with $\Pi_{1}$ with canonical monomorphism $\iota$ and canonical epimorphism $\pi$, we write $\iota=\iota^{E}:=\iota$ and $\pi=\pi^{E}:=\pi$. 
We suppose given a Grothendieck universe $\mathfrak{U}$. A crossed module extension is said to be a $\mathfrak{U}$-crossed module extension if its underlying crossed module is a $\mathfrak{U}$-crossed module. The set of crossed module extensions in $\mathfrak{U}$ of $G$ with $M$ will be denoted by $\underline{\operatorname{Ext}^{2}}(G, M)=\underline{\operatorname{Ext}}_{\mathfrak{U}}^{2}(G, M)$.

By definition, we have $\pi_{0}(E) \cong \Pi_{0}$ and $\pi_{1}(E) \cong \Pi_{1}$ for every crossed module extension $E$ of $\Pi_{0}$ with $\Pi_{1}$. Conversely, given an arbitrary crossed module $V$, we have the crossed module extension

$$
\pi_{1}(V) \stackrel{\text { inc }}{\longrightarrow} \operatorname{Mp} V \stackrel{\mu}{\longrightarrow} \operatorname{Gp} V \stackrel{\text { quo }}{\longrightarrow} \pi_{0}(V),
$$

again denoted by $V$. That is, the canonical monomorphism of $V$ is $\iota^{V}=\operatorname{inc}^{\pi_{1}(V)}$, and the canonical epimorphism is $\pi^{V}=$ quo $^{\pi_{0}(V)}$.

We let $E$ and $\tilde{E}$ be crossed module extensions of $\Pi_{0}$ with $\Pi_{1}$. An extension equivalence from $E$ to $\tilde{E}$ is a morphism of crossed modules $\varphi: E \rightarrow \tilde{E}$ such that $\iota^{\tilde{E}}=\iota^{E}(\operatorname{Mp} \varphi)$ and $\pi^{E}=(\operatorname{Gp} \varphi) \pi^{\tilde{E}}$.

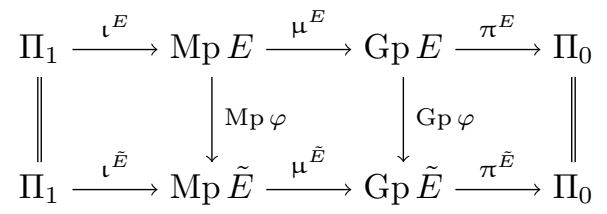

We suppose given a Grothendieck universe $\mathfrak{U}$ and we let $\approx=\approx_{\mathfrak{U}}$ be the equivalence relation on $\underline{\operatorname{Ext}}_{\mathfrak{U}}^{2}\left(\Pi_{0}, \Pi_{1}\right)$ generated by the following relation: Given extensions $E, \tilde{E} \in$ $\underline{\operatorname{Ext}}_{\mathfrak{U}}^{2}\left(\Pi_{0}, \Pi_{1}\right)$, the extension $E$ is in relation to the extension $\tilde{E}$ if there exists an extension equivalence $E \rightarrow \tilde{E}$. Given $\mathfrak{U}$-crossed module extensions $E$ and $\tilde{E}$ with $E \approx \tilde{E}$, we say that $E$ and $\tilde{E}$ are extension equivalent. The set of equivalence classes of $\mathfrak{U}$-crossed module extensions of $\Pi_{0}$ with $\Pi_{1}$ with respect to $\approx_{\mathfrak{U}}$ is denoted by $\operatorname{Ext}^{2}\left(\Pi_{0}, \Pi_{1}\right)=\operatorname{Ext}_{\mathfrak{U}}^{2}\left(\Pi_{0}, \Pi_{1}\right):=\operatorname{Ext}_{\mathfrak{U}}^{2}\left(\Pi_{0}, \Pi_{1}\right) / \approx_{\mathfrak{U}}$, and an element of $\operatorname{Ext}^{2}\left(\Pi_{0}, \Pi_{1}\right)$ is said to be a $\mathfrak{U}$-crossed module extension class of $\Pi_{0}$ with $\Pi_{1}$.

The following theorem appeared in various guises, see MAC LANE [25] and RATCLIFFE [28, th. 9.4]. It has been generalised to crossed complexes by HoLT [17, th. 4.5] and, independently, HuebschmanN [18, p. 310]. Moreover, there is a version for $n$-cat groups given by LODAY [23, th. 4.2].

Theorem. There is a bijection between the set of crossed module extension classes $\operatorname{Ext}_{\mathfrak{U}}^{2}\left(\Pi_{0}, \Pi_{1}\right)$ and the third cohomology group $\mathrm{H}^{3}\left(\Pi_{0}, \Pi_{1}\right)$, where $\mathfrak{U}$ is supposed to be a Grothendieck universe containing an infinite set.

This theorem can also be shown by arguments due to EILENBERG and MAC LANE, see $[\mathbf{1 3}$, sec. 7, sec. 9] and [24, sec. 7]. A detailed proof following these arguments, using the language of crossed modules, can be found in the manuscript [31], where a bijection $\operatorname{Ext}_{\mathfrak{U}}^{2}\left(\Pi_{0}, \Pi_{1}\right) \rightarrow \mathrm{H}^{3}\left(\Pi_{0}, \Pi_{1}\right),[E]_{\approx_{\mathfrak{U}}} \mapsto \mathrm{z}_{E}^{3} \mathrm{~B}^{3}\left(\Pi_{0}, \Pi_{1}\right)$ is explicitly constructed. This construction is used throughout section 4 . The inverse bijection $z^{3} \mathrm{~B}^{3}\left(\Pi_{0}, \Pi_{1}\right) \mapsto$ $\left[\mathrm{E}\left(z^{3}\right)\right]_{\approx_{\mathfrak{U}}}$ is used in corollary 4.10 . We give a sketch of these constructions.

Given pointed sets $X_{i}$ for $i \in I$ and $Y$, where $I$ is supposed to be an index set, let us call a map $f: X_{i \in I} X_{i} \rightarrow Y$ componentwise pointed if $\left(x_{i}\right)_{i \in I} f=*$ for all $\left(x_{i}\right)_{i \in I} \in \mathrm{X}_{i \in I} X_{i}$ with $x_{i}=*$ for some $i \in I$. So in particular, interpreting groups as pointed sets in the usual way, a 3-cochain $c^{3} \in \mathrm{Ch}^{3}\left(\Pi_{0}, \Pi_{1}\right)$ is componentwise 
pointed if it fulfills $(q, p, 1) c^{3}=(q, 1, p) c^{3}=(1, q, p) c^{3}=1$ for all $p, q \in \Pi_{0}$. The set of componentwise pointed 3-cochains of $\Pi_{0}$ with coefficients in $\Pi_{1}$ will be denoted by $\mathrm{Ch}_{\mathrm{cpt}}^{3}\left(\Pi_{0}, \Pi_{1}\right)$, the set of componentwise pointed 3 -cocycles by $\mathrm{Z}_{\mathrm{cpt}}^{3}\left(\Pi_{0}, \Pi_{1}\right):=$ $\mathrm{Z}^{3}\left(\Pi_{0}, \Pi_{1}\right) \cap \mathrm{Ch}_{\mathrm{cpt}}^{3}\left(\Pi_{0}, \Pi_{1}\right)$, the set of componentwise pointed 3-coboundaries by $\mathrm{B}_{\mathrm{cpt}}^{3}\left(\Pi_{0}, \Pi_{1}\right):=\mathrm{B}^{3}\left(\Pi_{0}, \Pi_{1}\right) \cap \mathrm{Ch}_{\mathrm{cpt}}^{3}\left(\Pi_{0}, \Pi_{1}\right)$ and the set of componentwise pointed 3 -cohomology classes by $\mathrm{H}_{\mathrm{cpt}}^{3}\left(\Pi_{0}, \Pi_{1}\right):=\mathrm{Z}_{\mathrm{cpt}}^{3}\left(\Pi_{0}, \Pi_{1}\right) / \mathrm{B}_{\text {cpt }}^{3}\left(\Pi_{0}, \Pi_{1}\right)$. With these notations, we have $\mathrm{H}^{3}\left(\Pi_{0}, \Pi_{1}\right) \cong \mathrm{H}_{\text {cpt }}^{3}\left(\Pi_{0}, \Pi_{1}\right)$. Analogously in other dimensions, cf. for example [31, cor. (3.7)].

We suppose given a crossed module extension $E$ of $\Pi_{0}$ with $\Pi_{1}$. First, we choose a lift of $\operatorname{id}_{\Pi_{0}}$ along the underlying pointed map of $\pi$, that is, a pointed map $Z^{1}: \Pi_{0} \rightarrow$ Gp $E$ with $Z^{1} \pi=\operatorname{id}_{\Pi_{0}}$. We obtain the componentwise pointed map

$$
\mathrm{z}^{2}=\mathrm{z}_{E, Z^{1}}^{2}: \Pi_{0} \times \Pi_{0} \rightarrow \operatorname{Im} \mu,(q, p) \mapsto\left(q Z^{1}\right)\left(p Z^{1}\right)\left((q p) Z^{1}\right)^{-1}
$$

fulfilling the non-abelian 2-cocycle condition $(r, q) \mathrm{z}^{2}(r q, p) \mathrm{z}^{2}=r Z^{1}\left((q, p) \mathrm{z}^{2}\right)(r, q p) \mathrm{z}^{2}$ for $p, q, r \in \Pi_{0}$. Next, we choose a componentwise pointed lift of $z^{2}$ along $\left.\mu\right|^{\operatorname{Im} \mu}$, that is, a componentwise pointed map $Z^{2}: \Pi_{0} \times \Pi_{0} \rightarrow \mathrm{Mp} E$ with $Z^{2}\left(\left.\mu\right|^{\operatorname{Im} \mu}\right)=\mathrm{z}^{2}$. This leads to the map

$$
\begin{aligned}
\mathrm{z}^{3}= & \mathrm{z}_{E,\left(Z^{2}, Z^{1}\right)}: \Pi_{0} \times \Pi_{0} \times \Pi_{0} \rightarrow \Pi_{1}, \\
& (r, q, p) \mapsto\left((r, q) Z^{2}(r q, p) Z^{2}\left((r, q p) Z^{2}\right)^{-1}\left(r Z^{1}\left((q, p) Z^{2}\right)\right)^{-1}\right)\left(\left.\iota\right|^{\operatorname{Im} \iota}\right)^{-1},
\end{aligned}
$$

which is shown to be a componentwise pointed 3-cocycle of $\Pi_{0}$ with coefficients in $\Pi_{1}$, that is, an element of $Z_{\mathrm{cpt}}^{3}\left(\Pi_{0}, \Pi_{1}\right)$. One shows that the cohomology class of $\mathrm{z}^{3}$ is independent from the choices of $Z^{1}, Z^{2}$ and the representative $E$ in its extension class.

A pair $\left(Z^{2}, Z^{1}\right)$ of componentwise pointed maps $Z^{1}: \Pi_{0} \rightarrow \mathrm{Gp} E$ and $Z^{2}: \Pi_{0} \times \Pi_{0}$ $\rightarrow \mathrm{Mp} E$ such that $Z^{1} \pi=\mathrm{id}_{\Pi_{0}}$ and $Z^{2}\left(\left.\mu\right|^{\operatorname{Im} \mu}\right)=\mathrm{z}^{2}$ is called a lifting system for $E$. Moreover, a pair $\left(s^{1}, s^{0}\right)$ of pointed maps $s^{0}: \Pi_{0} \rightarrow \operatorname{Gp} E$ and $s^{1}: \operatorname{Im} \mu \rightarrow \operatorname{Mp} E$ such that $s^{0} \pi=\mathrm{id}_{\Pi_{0}}$ and $s^{1}\left(\left.\mu\right|^{\operatorname{Im} \mu}\right)=\mathrm{id}_{\operatorname{Im} \mu}$ is said to be a section system for $E$. Every section system $\left(s^{1}, s^{0}\right)$ for $E$ provides a lifting system $\left(Z^{2}, Z^{1}\right)$ for $E$ by setting $Z^{1}:=s^{0}$ and $Z^{2}:=\mathrm{z}_{E, s^{0}}^{2} s^{1}$, called the lifting system coming from $\left(s^{1}, s^{0}\right)$. The $3-$ cocycle $\mathrm{z}^{3} \in \mathrm{Z}_{\mathrm{cpt}}^{3}\left(\Pi_{0}, \Pi_{1}\right)$ constructed as indicated above will be called the 3-cocycle of $E$ with respect to $\left(Z^{2}, Z^{1}\right)$. If $\left(Z^{2}, Z^{1}\right)$ comes from a section system $\left(s^{1}, s^{0}\right)$, we also write $\mathrm{z}^{3}=\mathrm{z}_{E,\left(s^{1}, s^{0}\right)}^{3}:=\mathrm{z}_{E,\left(Z^{2}, Z^{1}\right)}^{3}$ and call this the 3 -cocycle of $E$ with respect to $\left(s^{1}, s^{0}\right)$. Finally, we let $\mathrm{cl}(E):=\mathrm{z}^{3} \mathrm{~B}_{\mathrm{cpt}}^{3}\left(\Pi_{0}, \Pi_{1}\right)$.

Conversely, for a componentwise pointed 3-cocycle $z^{3} \in \mathrm{Z}_{\mathrm{cpt}}^{3}\left(\Pi_{0}, \Pi_{1}\right)$, the standard extension of $\Pi_{0}$ with $\Pi_{1}$ with respect to $z^{3}$ is constructed as follows.

We let $F$ be a free group on the underlying pointed set of $\Pi_{0}$ with basis $s^{0}=Z^{1}: \Pi_{0} \rightarrow F$, that is, $F$ is a free group on the set $\Pi_{0} \backslash\{1\}$ and $s^{0}$ maps $x \in \Pi_{0} \backslash\{1\}$ to the corresponding generator $x s^{0} \in F$, and $1 s^{0}=1$. We let $\pi: F \rightarrow \Pi_{0}$ be induced by $\operatorname{id}_{\Pi_{0}}: \Pi_{0} \rightarrow \Pi_{0}$. The basis $s^{0}$ is a section of the underlying pointed map of $\pi$. We let $z^{2}: \Pi_{0} \times \Pi_{0} \rightarrow \operatorname{Ker} \pi,(q, p) \mapsto\left(q s^{0}\right)\left(p s^{0}\right)\left((q p) s^{0}\right)^{-1}$. We let $\iota: \Pi_{1} \rightarrow$ $\Pi_{1} \times \operatorname{Ker} \pi, m \mapsto(m, 1)$ and $\mu: \Pi_{0} \times \operatorname{Ker} \pi \rightarrow F,(m, f) \mapsto f$. We let $s^{1}: \operatorname{Ker} \pi \rightarrow$ $\Pi_{0} \times \operatorname{Ker} \pi, f \mapsto(1, f)$ and we let $Z^{2}: \Pi_{0} \times \Pi_{0} \rightarrow \Pi_{1} \times \operatorname{Ker} \pi$ be given by $Z^{2}:=z^{2} s^{1}$. The direct product $\Pi_{1} \times \operatorname{Ker} \pi$ is generated by $\operatorname{Im} \iota \cup \operatorname{Im} Z^{2}$ and carries the structure of an $F$-module uniquely determined on this set of generators by $r Z^{1}(k \iota):=\left({ }^{r} k\right) \iota$ for 
$k \in \Pi_{1}, r \in \Pi_{0}$, and $r Z^{1}\left((q, p) Z^{2}\right):=\left((r, q, p) z^{3} \iota\right)^{-1}\left((r, q) Z^{2}\right)\left((r q, p) Z^{2}\right)\left((r, q p) Z^{2}\right)^{-1}$ for $p, q, r \in G$.

These data define the standard extension $\mathrm{E}\left(z^{3}\right)$ and the standard section system $\left(\mathrm{s}_{z^{3}}^{1}, \mathrm{~s}_{z^{3}}^{0}\right)$ for $\mathrm{E}\left(z^{3}\right)$ : The group part of $\mathrm{E}\left(z^{3}\right)$ is given by $\operatorname{Gp} \mathrm{E}\left(z^{3}\right):=F$, the module part is given by $\operatorname{MpE}\left(z^{3}\right):=M \times \operatorname{Ker} \pi$ and the structure morphism is given by $\mu^{\mathrm{E}\left(z^{3}\right)}:=\mu$. We have the canonical monomorphism $\iota^{\mathrm{E}\left(z^{3}\right)}:=\iota$ and the canonical epimorphism $\pi^{\mathrm{E}\left(z^{3}\right)}:=\pi$. The section system $\left(\mathrm{s}_{z^{3}}^{1}, \mathrm{~s}_{z^{3}}^{0}\right)$ is defined by $\mathrm{s}_{z^{3}}^{0}:=s^{0}$ and $\mathrm{s}_{z^{3}}^{1}:=s^{1}$.

By construction, the 3 -cocycle of $\mathrm{E}\left(z^{3}\right)$ with respect to the section system $\left(\mathrm{s}_{z^{3}}^{1}, \mathrm{~s}_{z^{3}}^{0}\right)$ is $z^{3}$. In particular, $\operatorname{cl}\left(\mathrm{E}\left(z^{3}\right)\right)=z^{3} \mathrm{~B}_{\mathrm{cpt}}^{3}(G, M)$.

\section{Low dimensional cohomology of a simplicial group}

In this section, we will show that the zeroth cohomology group of a simplicial group depends only on the coefficient module, that the first cohomology group depends only on the group segment and that the second cohomology group depends only on the crossed module segment.

Our results shall be achieved by means of calculations with analysed cocycles and coboundaries in low dimensions. Therefore, we restate their definitions explicitly.

\section{Working base 3.1 .}

(a) We suppose given a simplicial group $G$ and an abelian $\pi_{0}(G)$-module $M$. The analysed cochain complex $\mathrm{Ch}_{\mathrm{an}}(G, M)$ starts with the following entries. $\left.{ }^{6}\right)$

$$
\begin{aligned}
\mathrm{Ch}_{\mathrm{an}}^{0}(G, M)= & \operatorname{Map}(\{1\}, M), \\
\mathrm{Ch}_{\mathrm{an}}^{1}(G, M)= & \operatorname{Map}\left(\mathrm{M}_{0} G, M\right), \\
\mathrm{Ch}_{\mathrm{an}}^{2}(G, M)= & \operatorname{Map}\left(\mathrm{M}_{1} G \times \mathrm{M}_{0} G \times \mathrm{M}_{0} G, M\right), \\
\mathrm{Ch}_{\mathrm{an}}^{3}(G, M)= & \operatorname{Map}\left(\mathrm{M}_{2} G \times \mathrm{M}_{1} G \times \mathrm{M}_{1} G \times \mathrm{M}_{0} G\right. \\
& \left.\times \mathrm{M}_{1} G \times \mathrm{M}_{0} G \times \mathrm{M}_{0} G, M\right) .
\end{aligned}
$$

The differentials are given by

$$
\left(g_{0}\right)(c \partial)=1 c-g_{0} \mathrm{~B}_{0} \mathrm{M} G \cdot 1 c
$$

for $g_{0} \in \mathrm{M}_{0} G, c \in \mathrm{Ch}_{\mathrm{an}}^{0}(G, M)$,

$$
\left(g_{1}, h_{0}, g_{0}\right)(c \partial)=\left(g_{1} h_{0}\right) c-\left(h_{0} g_{0}\right) c+h_{0} \mathrm{~B}_{0} \mathrm{M} G \cdot\left(g_{0}\right) c
$$

for $g_{0}, h_{0} \in \mathrm{M}_{0} G, g_{1} \in \mathrm{M}_{1} G, c \in \mathrm{Ch}_{\text {an }}^{1}(G, M)$, and

$$
\begin{aligned}
& \left(g_{2}, k_{1}, h_{1}, k_{0}, g_{1}, h_{0}, g_{0}\right)(c \partial) \\
& \quad=\left(\left(g_{2} \partial\right) k_{1},\left(h_{1} \partial\right) k_{0},\left(g_{1} \partial\right) h_{0}\right) c-\left(k_{1} h_{1}, k_{0}, h_{0} g_{0}\right) c+\left(h_{1}{ }^{k_{0} \mathrm{~s}_{0}} g_{1}, k_{0} h_{0}, g_{0}\right) c \\
& \quad-k_{0} \mathrm{~B}_{0} \mathrm{M} G \cdot\left(g_{1}, h_{0}, g_{0}\right) c
\end{aligned}
$$

for $g_{0}, h_{0}, k_{0} \in \mathrm{M}_{0} G, g_{1}, h_{1}, k_{1} \in \mathrm{M}_{1} G, g_{2} \in \mathrm{M}_{2} G, c \in \mathrm{Ch}_{\text {an }}^{2}(G, M)$.

\footnotetext{
${ }^{6}$ To simplify notation, we identify $\left(\mathrm{M}_{1} G \times \mathrm{M}_{0} G\right) \times\left(\mathrm{M}_{0} G\right)$ with $\mathrm{M}_{1} G \times \mathrm{M}_{0} G \times \mathrm{M}_{0} G$, etc.
} 
(b) We suppose given a crossed module $V$ and an abelian $\pi_{0}(V)$-module $M$. The cochain complex $\mathrm{Ch}(V, M)$ starts with the following entries.

$$
\begin{aligned}
& \operatorname{Ch}^{0}(V, M)=\operatorname{Map}(\{1\}, M), \\
& \operatorname{Ch}^{1}(V, M)=\operatorname{Map}(\operatorname{Gp} V, M), \\
& \operatorname{Ch}^{2}(V, M)=\operatorname{Map}(\operatorname{Mp} V \times \operatorname{Gp} V \times \operatorname{Gp} V, M), \\
& \operatorname{Ch}^{3}(V, M)=\operatorname{Map}(\operatorname{Mp} V \times \operatorname{Mp} V \times \operatorname{Gp} V \times \operatorname{Mp} V \times \operatorname{Gp} V \times \operatorname{Gp} V, M) .
\end{aligned}
$$

The differentials are given by

$$
(g)(c \partial)=1 c-g(\operatorname{Im} \mu) \cdot 1 c
$$

for $g \in \operatorname{Gp} V, c \in \mathrm{Ch}^{0}(V, M)$,

$$
(m, h, g)(c \partial)=(m h) c-(h g) c+h(\operatorname{Im} \mu) \cdot(g) c
$$

for $g, h \in \operatorname{Gp} V, m \in \operatorname{Mp} V, c \in \operatorname{Ch}^{1}(V, M)$, and

$$
\begin{aligned}
& (p, n, k, m, h, g)(c \partial) \\
& \quad=(p, n k, m h) c-(p n, k, h g) c+\left(n^{k} m, k h, g\right) c-k(\operatorname{Im} \mu) \cdot(m, h, g) c
\end{aligned}
$$

for $g, h, k \in \operatorname{Gp} V, m, n, p \in \operatorname{Mp} V, c \in \mathrm{Ch}^{2}(V, M)$.

Proof.

(a) We show how the differential $\partial: \mathrm{Ch}_{\text {an }}^{2}(G, M) \rightarrow \mathrm{Ch}_{\text {an }}^{3}(G, M)$ of the analysed cochain complex is computed using transport of structure, the easier lower dimensional cases are left to the reader.

The corresponding entries of the cochain complex are $\operatorname{Ch}^{2}(G, M)=$ $\operatorname{Map}\left(G_{1} \times G_{0}, M\right)$ and $\operatorname{Ch}^{3}(G, M)=\operatorname{Map}\left(G_{2} \times G_{1} \times G_{0}, M\right)$. Now the semidirect product decompositions of $G_{0}, G_{1}$ and $G_{2}$ are given by the isomorphisms

$$
\begin{aligned}
& \varphi_{0}: G_{0} \rightarrow \mathrm{M}_{0} G, g_{0} \mapsto g_{0}, \\
& \varphi_{0}^{-1}: \mathrm{M}_{0} G \rightarrow G_{0}, g_{0} \mapsto g_{0}, \\
& \varphi_{1}: G_{1} \rightarrow \mathrm{M}_{1} G \rtimes \mathrm{M}_{0} G, g_{1} \mapsto\left(g_{1}\left(g_{1} \mathrm{~d}_{1} \mathrm{~s}_{0}\right)^{-1}, g_{1} \mathrm{~d}_{1}\right), \\
& \varphi_{1}^{-1}: \mathrm{M}_{1} G \rtimes \mathrm{M}_{0} G \rightarrow G_{1},\left(g_{1}, g_{0}\right) \mapsto g_{1}\left(g_{0} \mathrm{~s}_{0}\right), \\
& \varphi_{2}: G_{2} \rightarrow\left(\mathrm{M}_{2} G \rtimes \mathrm{M}_{1} G\right) \rtimes\left(\mathrm{M}_{1} G \rtimes \mathrm{M}_{0} G\right), \\
& \quad g_{2} \mapsto\left(\left(g_{2}\left(g_{2} \mathrm{~d}_{2} \mathrm{~s}_{1}\right)^{-1}\left(g_{2} \mathrm{~d}_{2} \mathrm{~s}_{0}\right)\left(g_{2} \mathrm{~d}_{1} \mathrm{~s}_{0}\right)^{-1},\left(g_{2} \mathrm{~d}_{1}\right)\left(g_{2} \mathrm{~d}_{2}\right)^{-1}\right),\right. \\
& \left.\quad\left(\left(g_{2} \mathrm{~d}_{2}\right)\left(g_{2} \mathrm{~d}_{2} \mathrm{~d}_{1} \mathrm{~s}_{0}\right)^{-1}, g_{2} \mathrm{~d}_{2} \mathrm{~d}_{1}\right)\right), \\
& \varphi_{2}^{-1}:\left(\mathrm{M}_{2} G \rtimes \mathrm{M}_{1} G\right) \rtimes\left(\mathrm{M}_{1} G \rtimes \mathrm{M}_{0} G\right) \rightarrow G_{2}, \\
& \quad\left(\left(g_{2}, h_{1}\right),\left(g_{1}, g_{0}\right)\right) \mapsto g_{2}\left(h_{1} \mathrm{~s}_{0}\right)\left(g_{1} \mathrm{~s}_{1}\right)\left(g_{0} \mathrm{~s}_{0} \mathrm{~s}_{1}\right) .
\end{aligned}
$$

Moreover, the image $c^{\prime} \partial \in \mathrm{Ch}^{3}(G, M)$ of a 2-cochain $c^{\prime} \in \mathrm{Ch}^{2}(G, M)$ is defined by

$$
\begin{gathered}
\left(g_{2}, g_{1}, g_{0}\right)\left(c^{\prime} \partial\right)=\left(g_{2} \mathrm{~d}_{0}, g_{1} \mathrm{~d}_{0}\right) c^{\prime}-\left(g_{2} \mathrm{~d}_{1},\left(g_{1} \mathrm{~d}_{1}\right) g_{0}\right) c^{\prime}+\left(\left(g_{2} \mathrm{~d}_{2}\right) g_{1}, g_{0}\right) c^{\prime} \\
-\left(g_{2} \mathrm{~d}_{2} \mathrm{~d}_{1} \mathrm{~B}_{0} \mathrm{M} G\right)\left(g_{1}, g_{0}\right) c^{\prime} .
\end{gathered}
$$


Hence we obtain

$$
\begin{aligned}
\mathrm{Ch}_{\text {an }}^{2}(G, M)= & \operatorname{Map}\left(\left(\mathrm{M}_{1} G \times \mathrm{M}_{0} G\right) \times \mathrm{M}_{0} G, M\right), \\
\mathrm{Ch}_{\text {an }}^{3}(G, M)= & \operatorname{Map}\left(\left(\mathrm{M}_{2} G \times \mathrm{M}_{1} G \times \mathrm{M}_{1} G \times \mathrm{M}_{0} G\right)\right. \\
& \left.\times\left(\mathrm{M}_{1} G \times \mathrm{M}_{0} G\right) \times \mathrm{M}_{0} G, M\right),
\end{aligned}
$$

and, using the isomorphisms $\varphi_{i}$ for $i \in\{0,1,2\}$, the image $c \partial \in \mathrm{Ch}_{\text {an }}^{3}(G, M)$ of an analysed 2-cochain $c \in \mathrm{Ch}_{\text {an }}^{2}(G, M)$ is given by

$$
c \partial=\left(\varphi_{2}^{-1} \times \varphi_{1}^{-1} \times \varphi_{0}^{-1}\right)\left(\left(\left(\varphi_{1} \times \varphi_{0}\right) c\right) \partial\right),
$$

that is, we have

$$
\begin{aligned}
\left(\left(g_{2}, k_{1},\right.\right. & \left.\left.h_{1}, k_{0}\right),\left(g_{1}, h_{0}\right), g_{0}\right)(c \partial) \\
= & \left(\left(g_{2}, k_{1}, h_{1}, k_{0}\right) \varphi_{2}^{-1},\left(g_{1}, h_{0}\right) \varphi_{1}^{-1}, g_{0} \varphi_{0}^{-1}\right)\left(\left(\left(\varphi_{1} \times \varphi_{0}\right) c\right) \partial\right) \\
= & \left(g_{2}\left(k_{1} \mathrm{~s}_{0}\right)\left(h_{1} \mathrm{~s}_{1}\right)\left(k_{0} \mathrm{~s}_{0} \mathrm{~s}_{1}\right), g_{1}\left(h_{0} \mathrm{~s}_{0}\right), g_{0}\right)\left(\left(\left(\varphi_{1} \times \varphi_{0}\right) c\right) \partial\right) \\
= & \left(\left(g_{2}\left(k_{1} \mathrm{~s}_{0}\right)\left(h_{1} \mathrm{~s}_{1}\right)\left(k_{0} \mathrm{~s}_{0} \mathrm{~s}_{1}\right)\right) \mathrm{d}_{0},\left(g_{1}\left(h_{0} \mathrm{~s}_{0}\right)\right) \mathrm{d}_{0}\right)\left(\left(\varphi_{1} \times \varphi_{0}\right) c\right) \\
& -\left(\left(g_{2}\left(k_{1} \mathrm{~s}_{0}\right)\left(h_{1} \mathrm{~s}_{1}\right)\left(k_{0} \mathrm{~s}_{0} \mathrm{~s}_{1}\right)\right) \mathrm{d}_{1},\left(\left(g_{1}\left(h_{0} \mathrm{~s}_{0}\right)\right) \mathrm{d}_{1}\right) g_{0}\right)\left(\left(\varphi_{1} \times \varphi_{0}\right) c\right) \\
& +\left(\left(\left(g_{2}\left(k_{1} \mathrm{~s}_{0}\right)\left(h_{1} \mathrm{~s}_{1}\right)\left(k_{0} \mathrm{~s}_{0} \mathrm{~s}_{1}\right)\right) \mathrm{d}_{2}\right)\left(g_{1}\left(h_{0} \mathrm{~s}_{0}\right)\right), g_{0}\right)\left(\left(\varphi_{1} \times \varphi_{0}\right) c\right) \\
& -\left(g_{2}\left(k_{1} \mathrm{~s}_{0}\right)\left(h_{1} \mathrm{~s}_{1}\right)\left(k_{0} \mathrm{~s}_{0} \mathrm{~s}_{1}\right)\right) \mathrm{d}_{2} \mathrm{~d}_{1} \mathrm{~B}_{0} \mathrm{M} G \cdot\left(g_{1}\left(h_{0} \mathrm{~s}_{0}\right), g_{0}\right)\left(\left(\varphi_{1} \times \varphi_{0}\right) c\right) \\
= & \left.\left(\left(g_{2} \partial\right) k_{1}\left(h_{1} \partial \mathrm{s}_{0}\right)\left(k_{0} \mathrm{~s}_{0}\right)\right) \varphi_{1},\left(\left(g_{1} \partial\right) h_{0}\right) \varphi_{0}\right) c \\
& \quad-\left(\left(k_{1} h_{1}\left(k_{0} \mathrm{~s}_{0}\right)\right) \varphi_{1},\left(h_{0} g_{0}\right) \varphi_{0}\right) c+\left(\left(h_{1}\left(k_{0} \mathrm{~s}_{0}\right) g_{1}\left(h_{0} \mathrm{~s}_{0}\right)\right) \varphi_{1}, g_{0} \varphi_{0}\right) c \\
& \quad-k_{0} \mathrm{~B}_{0} \mathrm{M} G \cdot\left(\left(g_{1}\left(h_{0} \mathrm{~s}_{0}\right)\right) \varphi_{1}, g_{0} \varphi_{0}\right) c \\
= & \left.\left(\left(g_{2} \partial\right) k_{1}\left(h_{1} \partial \mathrm{s}_{0}\right)\left(k_{0} \mathrm{~s}_{0}\right)\left(k_{0} \mathrm{~s}_{0}\right)^{-1}\left(h_{1} \partial \mathrm{s}_{0}\right)^{-1},\left(h_{1} \partial\right) k_{0}\right),\left(g_{1} \partial\right) h_{0}\right) c \\
& \quad-\left(\left(k_{1} h_{1}\left(k_{0} \mathrm{~s}_{0}\right)\left(k_{0} \mathrm{~s}_{0}\right)^{-1}, k_{0}\right), h_{0} g_{0}\right) c \\
& +\left(\left(h_{1}\left(k_{0} \mathrm{~s}_{0}\right) g_{1}\left(h_{0} \mathrm{~s}_{0}\right)\left(h_{0} \mathrm{~s}_{0}\right)^{-1}\left(k_{0} \mathrm{~s}_{0}\right)^{-1}, k_{0} h_{0}\right), g_{0}\right) c \\
& \quad-k_{0} \mathrm{~B}_{0} \mathrm{M} G \cdot\left(\left(g_{1}\left(h_{0} \mathrm{~s}_{0}\right)\left(h_{0} \mathrm{~s}_{0}\right)^{-1}, h_{0}\right), g_{0}\right) c \\
= & \left.\left(\left(g_{2} \partial\right) k_{1},\left(h_{1} \partial\right) k_{0}\right), g_{1} h_{0}\right) c-\left(\left(\left(k_{1} \partial\right) h_{1}, k_{0}\right), h_{0} g_{0}\right) c \\
& +\left(\left(h_{1} k_{0} g_{1}, k_{0} h_{0}\right), g_{0}\right) c-k_{0} \mathrm{~B}_{0} \mathrm{M} G \cdot\left(\left(g_{1}, h_{0}\right), g_{0}\right) c
\end{aligned}
$$

for $g_{0}, h_{0}, k_{0} \in \mathrm{M}_{0} G, g_{1}, h_{1}, k_{1} \in \mathrm{M}_{1} G, g_{2} \in \mathrm{M}_{2} G$.

(b) This follows from (a) and the definition of crossed module cohomology via Cosk $_{1}$, cf. section 2.11.

We immediately obtain the following result about the zeroth cohomology group, which states that it only depends on the module of coefficients (and therefore implicitly on the zeroth homotopy group by our choice of coefficients).

Proposition 3.2. Given a simplicial group $G$ and an abelian $\pi_{0}(G)$-module $M$, we have

$$
\mathrm{H}^{0}(G, M) \cong \mathrm{H}^{0}\left(\pi_{0}(G), M\right) \cong\left\{m \in M \mid p m=m \text { for all } p \in \pi_{0}(G)\right\} .
$$

Corollary 3.3. Given a crossed module $V$ and an abelian $\pi_{0}(V)$-module $M$, we have $\mathrm{H}^{0}(V, M) \cong \mathrm{H}^{0}\left(\pi_{0}(V), M\right) \cong\left\{m \in M \mid p m=m\right.$ for all $\left.p \in \pi_{0}(V)\right\}$. 
We suppose given a simplicial group $G$, an abelian group $A$ and $n \in\{0,1\}$. In propositions 3.5 and 3.13 , we will show that $\mathrm{H}^{n+1}(G, A) \cong \mathrm{H}^{n+1}\left(\operatorname{Trunc}^{n} G, A\right)$. Using homotopy theory of topological spaces, this can be seen as follows.

We consider the unit component $\varepsilon_{G}: G \rightarrow \operatorname{Cosk}_{n} \operatorname{Trunc}^{n} G$ of the adjunction Trunc $^{n} \dashv \operatorname{Cosk}_{n}$ and claim that $\pi_{k} \varepsilon_{G}$ is an isomorphism for $k \in[0, n]$, cf. section 2.5. If $n=0$, one reads off that Trunc ${ }^{0} \varepsilon_{G}$ is an isomorphism and hence $\pi_{0} \varepsilon_{G}$ is an isomorphism since $\pi_{0}=\operatorname{Trunc}^{0}$. If $n=1$, one reads off that $\operatorname{Gp}\left(\operatorname{Trunc}^{1} \varepsilon_{G}\right)$ and $\operatorname{Mp}\left(\operatorname{Trunc}^{1} \varepsilon_{G}\right)$ are isomorphisms, hence $\operatorname{Trunc}^{1} \varepsilon_{G}$ is an isomorphism and thus $\pi_{k} \varepsilon_{G}=\pi_{k}\left(\operatorname{Trunc}^{1} \varepsilon_{G}\right)$ are isomorphisms for $k \in[0,1]$, cf. [29, prop. (6.25)].

The canonical simplicial map $\mathrm{W} G \rightarrow \overline{\mathrm{W}} G$ is a Kan fibration with fiber $G$, and WG is contractible, see [16, ch. V, lem. 4.1, lem. 4.6]. Analogously for $\operatorname{Cosk}_{n} \operatorname{Trunc}^{n} G$, so the induced long exact homotopy sequence $[\mathbf{2 2}$, ch. VII, 4.1, 4.2, 5.3] shows that $\pi_{k}\left(\overline{\mathrm{W}} \varepsilon_{G}\right)$ are isomorphisms for $k \in[0, n+1]$. It follows that $\pi_{k}\left(\left|\overline{\mathrm{W}} \varepsilon_{G}\right|\right)$ are isomorphisms for $k \in[0, n+1]$, see [16, ch. I, prop. 11.1] and [22, ch. VII, 10.9]. The Whitehead theorem [3, ch. VII, th. 11.2 I(b)] provides isomorphisms $\mathrm{H}_{k}\left(\left|\overline{\mathrm{W}} \varepsilon_{G}\right|\right)$ for $k \in[0, n+1]$. The universal coefficient theorem [3, ch. V, cor. 7.2] yields isomorphisms $\mathrm{H}^{k}\left(\left|\overline{\mathrm{W}} \varepsilon_{G}\right|, A\right)$ for $k \in[0, n+1]$. Finally, $\mathrm{H}^{k}\left(\overline{\mathrm{W}} \varepsilon_{G}, A\right)$ are isomorphisms for $k \in[0, n+1]$ by $\left[\mathbf{2 0}\right.$, th. 6.3]. In particular, one obtains $\mathrm{H}^{n+1}(G, A) \cong$ $\mathrm{H}^{n+1}\left(\operatorname{Cosk}_{n} \operatorname{Trunc}^{n} G, A\right)=\mathrm{H}^{n+1}\left(\operatorname{Trunc}^{n} G, A\right)$, as desired.

However, we will not make use of these topological arguments. Following the overall intention of this article, we will give direct algebraic proofs of these results. Moreover, we will use proposition 3.11(b) several times in section 4 , in particular in the proofs of proposition 4.4 and proposition 4.7 .

Proposition 3.4. We suppose given a simplicial group $G$ and an abelian $\pi_{0}(G)$ module $M$. The first analysed cocycle group $\mathrm{Z}_{\mathrm{an}}^{1}(G, M)$ is the kernel of

$$
\operatorname{inc}^{\mathrm{Z}^{1}\left(\mathrm{M}_{0} G, M\right)} \mathrm{Ch}^{1}\left(\partial^{\mathrm{M} G}, M\right): \mathrm{Z}^{1}\left(\mathrm{M}_{0} G, M\right) \rightarrow \mathrm{Ch}^{1}\left(\mathrm{M}_{1} G, M\right),
$$

that is, we have

$$
\mathrm{Z}_{\text {an }}^{1}(G, M)=\left\{z_{0} \in \mathrm{Z}^{1}\left(\mathrm{M}_{0} G, M\right)\left|z_{0}\right|_{\mathrm{B}_{0} \mathrm{M} G}=0\right\} .
$$

Proof. For every element $z \in \mathrm{Z}_{\mathrm{an}}^{1}(G, M)$, we have

$$
\begin{aligned}
0 & =\left(1, h_{0}, g_{0}\right)\left(z \partial^{\mathrm{Ch}_{\text {an }}(G, M)}\right)=\left(h_{0}\right) z-\left(h_{0} g_{0}\right) z+h_{0} \mathrm{~B}_{0} \mathrm{M} G \cdot\left(g_{0}\right) z \\
& =\left(h_{0}, g_{0}\right)\left(z \partial^{\mathrm{Ch}\left(\mathrm{M}_{0} G, M\right)}\right)
\end{aligned}
$$

for all $g_{0}, h_{0} \in \mathrm{M}_{0} G$ as well as

$$
0=\left(g_{1}, 1,1\right)\left(z \partial^{\mathrm{Ch}_{\text {an }}(G, M)}\right)=\left(g_{1} \partial\right) z
$$

for all $g_{1} \in \mathrm{M}_{1} G$, that is, $\mathrm{Z}_{\mathrm{an}}^{1}(G, M) \subseteq \mathrm{Z}^{1}\left(\mathrm{M}_{0} G, M\right)$ and $\left.z\right|_{\mathrm{B}_{0} \mathrm{M} G}=0$. Conversely, given a 1-cocycle $z_{0} \in \mathrm{Z}^{1}\left(\mathrm{M}_{0} G, M\right)$ with $\left.z_{0}\right|_{\mathrm{B}_{0} \mathrm{M} G}=0$, it follows that

$$
\begin{aligned}
& \left(g_{1}, h_{0}, g_{0}\right)\left(z_{0} \partial^{\mathrm{Ch}_{\text {an }}(G, M)}\right)=\left(\left(g_{1} \partial\right) h_{0}\right) z_{0}-\left(h_{0} g_{0}\right) z_{0}+h_{0} \mathrm{~B}_{0} \mathrm{M} G \cdot\left(g_{0}\right) z_{0} \\
& \quad=\left(g_{1}\right) z_{0}+\left(g_{1} \partial\right) \mathrm{B}_{0} \mathrm{M} G \cdot\left(h_{0}\right) z_{0}-\left(h_{0} g_{0}\right) z_{0}+h_{0} \mathrm{~B}_{0} \mathrm{M} G \cdot\left(g_{0}\right) z_{0} \\
& \quad=\left(h_{0}\right) z_{0}-\left(h_{0} g_{0}\right) z_{0}+h_{0} \mathrm{~B}_{0} \mathrm{M} G \cdot\left(g_{0}\right) z_{0}=\left(h_{0}, g_{0}\right)\left(z_{0} \partial^{\mathrm{Ch}\left(\mathrm{M}_{0} G, M\right)}\right)=0
\end{aligned}
$$

for $g_{1} \in \mathrm{M}_{1} G, g_{0}, h_{0} \in \mathrm{M}_{0} G$, that is, $z_{0} \in \mathrm{Z}_{\text {an }}^{1}(G, M)$. Altogether, we have

$$
\mathrm{Z}_{\text {an }}^{1}(G, M)=\left\{z_{0} \in \mathrm{Z}^{1}\left(\mathrm{M}_{0} G, M\right)\left|z_{0}\right|_{\mathrm{B}_{0} \mathrm{M} G}=0\right\} .
$$


Recall that $\operatorname{Ch}\left(\operatorname{Trunc}^{0} G, M\right)=\mathrm{Ch}_{\mathrm{an}}\left(\operatorname{Cosk}_{0} \operatorname{Trunc}^{0} G, M\right)$ for every simplicial group $G$.

Proposition 3.5. Given a simplicial group $G$ and an abelian $\pi_{0}(G)$-module $M$, the unit component $\varepsilon_{G}: G \rightarrow \operatorname{Cosk}_{0} \operatorname{Trunc}^{0} G$ of the adjunction $\operatorname{Trunc}^{0} \dashv \operatorname{Cosk}_{0}$ induces an isomorphism

$$
\mathrm{Z}_{\mathrm{an}}^{1}\left(\varepsilon_{G}, M\right): \mathrm{Z}^{1}\left(\operatorname{Trunc}^{0} G, M\right) \rightarrow \mathrm{Z}_{\mathrm{an}}^{1}(G, M),
$$

which in turn induces isomorphisms $\mathrm{B}_{\mathrm{an}}^{1}\left(\varepsilon_{G}, M\right)$ and $\mathrm{H}_{\mathrm{an}}^{1}\left(\varepsilon_{G}, M\right)$. In particular, we have

$$
\mathrm{H}^{1}(G, M) \cong \mathrm{H}^{1}\left(\operatorname{Trunc}^{0} G, M\right) .
$$

Proof. We let $\pi: \mathrm{M}_{0} G \rightarrow \mathrm{M}_{0} G / \mathrm{B}_{0} \mathrm{M} G=\operatorname{Trunc}^{0} G$ denote the canonical epimorphism, cf. section 2.5. The induced group homomorphism $\mathrm{Z}_{\text {an }}^{1}\left(\varepsilon_{G}, M\right)$ is given by $\left(g_{0}\right)\left(z^{\prime} \mathrm{Z}_{\text {an }}^{1}\left(\varepsilon_{G}, M\right)\right)=\left(g_{0} \pi\right) z^{\prime}$ for $g_{0} \in \mathrm{M}_{0} G, z^{\prime} \in \mathrm{Z}^{1}\left(\operatorname{Trunc}^{0} G, M\right)$. Thus we have $z^{\prime} \mathrm{Z}_{\mathrm{an}}^{1}\left(\varepsilon_{G}, M\right)=0$ if and only if already $z^{\prime}=0$, that is, $\mathrm{Z}_{\mathrm{an}}^{1}\left(\varepsilon_{G}, M\right)$ is injective.

To show surjectivity, we suppose given an analysed 1-cochain $z \in \mathrm{Z}_{\mathrm{an}}^{1}(G, M)$. We choose a section of the underlying pointed map of $\pi$, that is, a pointed map $s: \operatorname{Trunc}^{0} G \rightarrow \mathrm{M}_{0} G$ with $s \pi=\operatorname{id}_{\operatorname{Trunc}^{0} G}$. Then $(q s)(p s)((q p) s)^{-1} \in \operatorname{Ker} \pi=\mathrm{B}_{0} \mathrm{M} G$ and therefore, by proposition 3.4,

$$
\begin{aligned}
((q s)(p s)) z & =\left((q s)(p s)((q p) s)^{-1}((q p) s)\right) z \\
& =\left((q s)(p s)((q p) s)^{-1}\right) z+\left((q s)(p s)((q p) s)^{-1}\right) \mathrm{B}_{0} \mathrm{M} G \cdot((q p) s) z \\
& =((q p) s) z
\end{aligned}
$$

for all $p, q \in \operatorname{Trunc}^{0} G$. Now the pointed map $z^{\prime}: \operatorname{Trunc}^{0} G \rightarrow M$ defined by $(p) z^{\prime}:=$ (ps) $z$ for $p \in \operatorname{Trunc}^{0} G$ is a 1 -cocycle in $\mathrm{Z}^{1}\left(\operatorname{Trunc}^{0} G, M\right)$ since

$$
\begin{aligned}
(q, p)\left(z^{\prime} \partial^{\mathrm{Ch}\left(\operatorname{Trunc}^{0} G, M\right)}\right) & =(q) z^{\prime}-(q p) z^{\prime}+q \cdot(p) z^{\prime} \\
& =(q s) z-((q p) s) z+q s \pi \cdot(p s) z \\
& =(q s) z-((q s)(p s)) z+(q s) \mathrm{B}_{0} \mathrm{M} G \cdot(p s) z \\
& =(1, q s, p s)\left(z \partial^{\mathrm{Ch}_{\text {an }}(G, M)}\right)=0
\end{aligned}
$$

for all $p, q \in \operatorname{Trunc}^{0} G$. Further, $g_{0}\left(g_{0} \pi s\right)^{-1} \in \operatorname{Ker} \pi=\mathrm{B}_{0} \mathrm{M} G$ implies, using proposition 3.4,

$$
\begin{aligned}
0 & =\left(g_{0}\left(g_{0} \pi s\right)^{-1}\right) z=\left(g_{0}\right) z+g_{0} \mathrm{~B}_{0} \mathrm{M} G \cdot\left(\left(g_{0} \pi s\right)^{-1}\right) z \\
& =\left(g_{0}\right) z+\left(g_{0} \pi s\right) \mathrm{B}_{0} \mathrm{M} G \cdot\left(\left(g_{0} \pi s\right)^{-1}\right) z \\
& =\left(g_{0}\right) z-\left(g_{0} \pi s\right) z+\left(\left(g_{0} \pi s\right)\left(g_{0} \pi s\right)^{-1}\right) z=\left(g_{0}\right) z-\left(g_{0}\right)\left(z^{\prime} \mathrm{Z}_{\mathrm{an}}^{1}\left(\varepsilon_{G}, M\right)\right)
\end{aligned}
$$

and therefore $\left(g_{0}\right)\left(z^{\prime} \mathrm{Z}_{\mathrm{an}}^{1}\left(\varepsilon_{G}, M\right)\right)=\left(g_{0}\right) z$ for all $g_{0} \in \mathrm{M}_{0} G$, that is, $z^{\prime} \mathrm{Z}_{\mathrm{an}}^{1}\left(\varepsilon_{G}, M\right)=z$. Thus $\mathrm{Z}_{\mathrm{an}}^{1}\left(\varepsilon_{G}, M\right)$ is surjective. Altogether, $\mathrm{Z}_{\mathrm{an}}^{1}\left(\varepsilon_{G}, M\right)$ is an isomorphism of abelian groups.

Now the injectivity of $\mathrm{Z}_{\mathrm{an}}^{1}\left(\varepsilon_{G}, M\right)$ implies the injectivity of the restriction $\mathrm{B}_{\text {an }}^{1}\left(\varepsilon_{G}, M\right)$. To show that this is also an isomorphism, it remains to show that for every analysed 1-coboundary $b \in \mathrm{B}_{\text {an }}^{1}(G, M)$, the 1-cocycle $b^{\prime} \in \mathrm{Z}^{1}\left(\operatorname{Trunc}^{0} G, M\right)$ 
given by $(p) b^{\prime}:=(p s) b$ for $p \in \operatorname{Trunc}^{0} G$ is in fact a 1-coboundary, that is, an element in $\mathrm{B}^{1}\left(\right.$ Trunc $\left.^{0} G, M\right)$. Indeed, given $b \in \mathrm{B}_{\text {an }}^{1}(G, M)$ and an analysed 0-cochain $c \in \mathrm{Ch}_{\text {an }}^{0}(G, M)$ with $b=c \partial^{\mathrm{Ch}_{\text {an }}(G, M)}$, it follows that

$$
(p) b^{\prime}=(p s) b=1 c-(p s) \mathrm{B}_{0} \mathrm{M} G \cdot 1 c=1 c-p \cdot 1 c=(p)\left(c \partial^{\mathrm{Ch}\left(\operatorname{Trunc}^{0} G, M\right)}\right)
$$

for all $p \in \operatorname{Trunc}^{0} G$ and hence $b^{\prime}=c \partial^{\operatorname{Ch}\left(\operatorname{Trunc}^{0} G, M\right)} \in \mathrm{B}^{1}\left(\operatorname{Trunc}^{0} G, M\right)$.

Thus we have shown that $\mathrm{Z}_{\mathrm{an}}^{1}\left(\varepsilon_{G}, M\right)$ and $\mathrm{B}_{\mathrm{an}}^{1}\left(\varepsilon_{G}, M\right)$ are isomorphisms, and hence $\mathrm{H}_{\mathrm{an}}^{1}\left(\varepsilon_{G}, M\right)$ is also an isomorphism. In particular, we have

$$
\mathrm{H}^{1}(G, M) \cong \mathrm{H}_{\mathrm{an}}^{1}(G, M) \cong \mathrm{H}^{1}\left(\operatorname{Trunc}^{0} G, M\right) .
$$

Corollary 3.6. Given a simplicial group $G$ and an abelian $\pi_{0}(G)$-module $M$, we have

$$
\mathrm{H}^{1}(G, M) \cong \mathrm{H}^{1}\left(\pi_{0}(G), M\right) .
$$

Corollary 3.7. Given a crossed module $V$ and an abelian $\pi_{0}(V)$-module $M$, we have

$$
\mathrm{H}^{1}(V, M) \cong \mathrm{H}^{1}\left(\pi_{0}(V), M\right) .
$$

We recall a simple fact of 2-cocycles of (ordinary) groups:

Remark 3.8. We let $G$ be a group and $M$ be an abelian $G$-module. For every 2cocycle $z \in \mathrm{Z}^{2}(G, M)$, we have $(g, 1) z=g \cdot(1,1) z$ and $(1, g) z=(1,1) z$ for all $g \in G$.

Proof. Given a 2-cocycle $z \in \mathrm{Z}^{2}(G, M)$, we have

$$
0=(g, 1,1)(z \partial)=(g, 1) z-(g, 1) z+(g, 1) z-g \cdot(1,1) z=(g, 1) z-g \cdot(1,1) z,
$$

that is, $(g, 1) z=g \cdot(1,1) z$, and

$$
0=(1,1, g)(z \partial)=(1,1) z-(1, g) z+(1, g) z-(1, g) z=(1,1) z-(1, g) z,
$$

that is, $(1, g) z=(1,1) z$ for all $g \in G$.

Corollary 3.9. We let $G$ be a group and $M$ be an abelian $G$-module. A 2-cocycle $z \in \mathrm{Z}^{2}(G, M)$ is componentwise pointed if and only if it is pointed.

To simplify our calculations, we give a bit more convenient description of the analysed 2-cocycles.

Definition 3.10 (Moore decomposition of analysed 2-cochains).

(a) We let $G$ be a simplicial group and $M$ be an abelian $\pi_{0}(G)$-module. Given an analysed 2-cochain $c \in \mathrm{Ch}_{\text {an }}^{2}(G, M)$, the 1-cochain $c_{\mathrm{M}_{1}} \in \mathrm{Ch}^{1}\left(\mathrm{M}_{1} G, M\right)$ defined by $\left(g_{1}\right) c_{\mathrm{M}_{1}}:=\left(g_{1}, 1,1\right) c$ for $g_{1} \in \mathrm{M}_{1} G$ is called the $\mathrm{M}_{1}$-part of $c$, and the 2cochain $c_{\mathrm{M}_{0}} \in \mathrm{Ch}^{2}\left(\mathrm{M}_{0} G, M\right)$ defined by $\left(h_{0}, g_{0}\right) c_{\mathrm{M}_{0}}:=\left(1, h_{0}, g_{0}\right) c$ for $g_{0}, h_{0} \in$ $\mathrm{M}_{0} G$ is called the $\mathrm{M}_{0}$-part of $c$.

(b) We let $V$ be a crossed module and $M$ be an abelian $\pi_{0}(V)$-module. Given a 2-cochain $c \in \mathrm{Ch}^{2}(V, M)$, we call the $\mathrm{M}_{1}$-part of $c$ also the module part of $c$ and write $c_{\mathrm{Mp}}:=c_{\mathrm{M}_{1}}$, and we call the $\mathrm{M}_{0}$-part of $c$ also the group part of $c$ and write $c_{\mathrm{Gp}}:=c_{\mathrm{M}_{0}}$. That is, $(m) c_{\mathrm{Mp}}=(m, 1,1) c$ for $m \in \operatorname{Mp} V$ and $(h, g) c_{\mathrm{Gp}}=$ $(1, h, g) c$ for $g, h \in \mathrm{Gp} V$. 


\section{Proposition 3.11.}

(a) We suppose given a simplicial group $G$ and an abelian $\pi_{0}(G)$-module $M$. An analysed 2-cochain $z \in \mathrm{Ch}_{\mathrm{an}}^{2}(G, M)$ is an analysed 2-cocycle if and only if it fulfills the following conditions.

(i) We have $\left(g_{1}, h_{0}, g_{0}\right) z=\left(g_{1}\right) z_{\mathrm{M}_{1}}-\left(g_{1} \partial, h_{0}\right) z_{\mathrm{M}_{0}}+\left(h_{0}, g_{0}\right) z_{\mathrm{M}_{0}}$ for $g_{1} \in \mathrm{M}_{1} G$, $g_{0}, h_{0} \in \mathrm{M}_{0} G$.

(ii) The $\mathrm{M}_{0}$-part $z_{\mathrm{M}_{0}}$ is a 2-cocycle of $\mathrm{M}_{0} G$ with coefficients in $M$, that is, $z_{\mathrm{M}_{0}} \in \mathrm{Z}^{2}\left(\mathrm{M}_{0} G, M\right)$.

(iii) We have $\left(h_{1} g_{1}\right) z_{\mathrm{M}_{1}}=\left(h_{1}\right) z_{\mathrm{M}_{1}}+\left(g_{1}\right) z_{\mathrm{M}_{1}}-\left(h_{1} \partial, g_{1} \partial\right) z_{\mathrm{M}_{0}}$ for $g_{1}, h_{1} \in \mathrm{M}_{1} G$.

(iv) We have $\left.{ }^{g_{0} \mathrm{~s}_{0}} g_{1}\right) z_{\mathrm{M}_{1}}=g_{0} \mathrm{~B}_{0} \mathrm{M} G \cdot\left(g_{1}\right) z_{\mathrm{M}_{1}}+\left({ }^{g_{0}}\left(g_{1} \partial\right), g_{0}\right) z_{\mathrm{M}_{0}}-\left(g_{0}, g_{1} \partial\right) z_{\mathrm{M}_{0}}$ for $g_{1} \in \mathrm{M}_{1} G, g_{0} \in \mathrm{M}_{0} G$.

(v) We have $\left(g_{2} \partial\right) z_{\mathrm{M}_{1}}=(1) z_{\mathrm{M}_{1}}$ for $g_{2} \in \mathrm{M}_{2} G$.

(b) We suppose given a crossed module $V$ and an abelian $\pi_{0}(V)$-module $M$. A 2cochain $z \in \mathrm{Ch}^{2}(V, M)$ is a 2-cocycle if and only if it fulfills the following conditions.

(i) We have $(m, h, g) z=(m) z_{\mathrm{Mp}}-(m, h) z_{\mathrm{Gp}}+(h, g) z_{\mathrm{Gp}}$ for $m \in \mathrm{Mp} V, g, h \in$ $\mathrm{Gp} V$.

(ii) The group part $z_{\mathrm{Gp}}$ is a 2-cocycle of $\mathrm{Gp} V$ with coefficients in $M$, that is, $z_{\mathrm{Gp}} \in \mathrm{Z}^{2}(\mathrm{Gp} V, M)$.

(iii) We have $(n m) z_{\mathrm{Mp}}=(n) z_{\mathrm{Mp}}+(m) z_{\mathrm{Mp}}-(n, m) z_{\mathrm{Gp}}$ for $m, n \in \mathrm{Mp} V$.

(iv) We have $\left({ }^{g} m\right) z_{\mathrm{Mp}}=g(\operatorname{Im} \mu) \cdot(m) z_{\mathrm{Mp}}+\left({ }^{g} m, g\right) z_{\mathrm{Gp}}-(g, m) z_{\mathrm{Gp}}$ for $m \in$ $\operatorname{Mp} V, g \in \operatorname{Gp} V$.

\section{Proof.}

(a) First, we suppose given an analysed 2-cocycle $z \in \mathrm{Z}_{\text {an }}^{2}(G, M)$. We verify the asserted formulas:

(ii) We have

$$
\begin{aligned}
0= & \left(1,1,1, k_{0}, 1, h_{0}, g_{0}\right)(z \partial) \\
= & \left(1, k_{0}, h_{0}\right) z-\left(1, k_{0}, h_{0} g_{0}\right) z+\left(1, k_{0} h_{0}, g_{0}\right) z-k_{0} \mathrm{~B}_{0} \mathrm{M} G \cdot\left(1, h_{0}, g_{0}\right) z \\
= & \left(k_{0}, h_{0}\right) z_{\mathrm{M}_{0}}-\left(k_{0}, h_{0} g_{0}\right) z_{\mathrm{M}_{0}}+\left(k_{0} h_{0}, g_{0}\right) z_{\mathrm{M}_{0}} \\
& \quad-k_{0} \mathrm{~B}_{0} \mathrm{M} G \cdot\left(h_{0}, g_{0}\right) z_{\mathrm{M}_{0}}
\end{aligned}
$$

for $g_{0}, h_{0}, k_{0} \in \mathrm{M}_{0} G$, that is, $z_{\mathrm{M}_{0}} \in \mathrm{Z}^{2}\left(\mathrm{M}_{0} G, M\right)$.

(i) First, we prove the formula for $h_{0}=1$, then for $g_{0}=1$ and finally for the general case.

We have

$$
\begin{aligned}
0 & =\left(1, g_{1}, 1,1,1, g_{0}, g_{0}^{-1}\right)(z \partial) \\
& =\left(g_{1}, 1, g_{0}\right) z-\left(g_{1}, 1,1\right) z+\left(1, g_{0}, g_{0}^{-1}\right) z-\left(1, g_{0}, g_{0}^{-1}\right) z \\
& =\left(g_{1}, 1, g_{0}\right) z-\left(g_{1}\right) z_{\mathrm{M}_{1}},
\end{aligned}
$$

that is, $\left(g_{1}, 1, g_{0}\right) z=\left(g_{1}\right) z_{\mathrm{M}_{1}}$ for $g_{1} \in \mathrm{M}_{1} G, g_{0} \in \mathrm{M}_{0} G$. 
Next, we obtain

$$
\begin{aligned}
0 & =\left(1,1, g_{1}, 1,1, h_{0}, 1\right)(z \partial) \\
& =\left(1, g_{1} \partial, h_{0}\right) z-\left(g_{1}, 1, h_{0}\right) z+\left(g_{1}, h_{0}, 1\right) z-\left(1, h_{0}, 1\right) z \\
& =\left(g_{1} \partial, h_{0}\right) z_{\mathrm{M}_{0}}-\left(g_{1}\right) z_{\mathrm{M}_{1}}+\left(g_{1}, h_{0}, 1\right) z-\left(h_{0}, 1\right) z_{\mathrm{M}_{0}},
\end{aligned}
$$

that is, $\left(g_{1}, h_{0}, 1\right) z=\left(g_{1}\right) z_{\mathrm{M}_{1}}-\left(g_{1} \partial, h_{0}\right) z_{\mathrm{M}_{0}}+\left(h_{0}, 1\right) z_{\mathrm{M}_{0}}$ for $g_{1} \in \mathrm{M}_{1} G$, $h_{0} \in \mathrm{M}_{0} G$.

Finally, we get, using (ii) and remark 3.8,

$$
\begin{aligned}
0= & \left(1, g_{1}, 1, h_{0}, 1,1, g_{0}\right)(z \partial) \\
= & \left(g_{1}, h_{0}, 1\right) z-\left(g_{1}, h_{0}, g_{0}\right) z+\left(1, h_{0}, g_{0}\right) z-h_{0} \mathrm{~B}_{0} \mathrm{M} G \cdot\left(1,1, g_{0}\right) z \\
= & \left(g_{1}\right) z_{\mathrm{M}_{1}}-\left(g_{1} \partial, h_{0}\right) z_{\mathrm{M}_{0}}+\left(h_{0}, 1\right) z_{\mathrm{M}_{0}}-\left(g_{1}, h_{0}, g_{0}\right) z+\left(h_{0}, g_{0}\right) z_{\mathrm{M}_{0}} \\
& \quad-h_{0} \mathrm{~B}_{0} \mathrm{M} G \cdot\left(1, g_{0}\right) z_{\mathrm{M}_{0}} \\
= & \left(g_{1}\right) z_{\mathrm{M}_{1}}-\left(g_{1} \partial, h_{0}\right) z_{\mathrm{M}_{0}}-\left(g_{1}, h_{0}, g_{0}\right) z+\left(h_{0}, g_{0}\right) z_{\mathrm{M}_{0}}
\end{aligned}
$$

that is, $\left(g_{1}, h_{0}, g_{0}\right) z=\left(g_{1}\right) z_{\mathrm{M}_{1}}-\left(g_{1} \partial, h_{0}\right) z_{\mathrm{M}_{0}}+\left(h_{0}, g_{0}\right) z_{\mathrm{M}_{0}}$ for $g_{1} \in \mathrm{M}_{1} G$, $g_{0}, h_{0} \in \mathrm{M}_{0} G$.

(iii) We have

$$
\begin{aligned}
0 & =\left(1,1, h_{1}, 1, g_{1}, 1,1\right)(z \partial) \\
& =\left(1, h_{1} \partial, g_{1} \partial\right) z-\left(h_{1}, 1,1\right) z+\left(h_{1} g_{1}, 1,1\right) z-\left(g_{1}, 1,1\right) z \\
& =\left(h_{1} \partial, g_{1} \partial\right) z_{\mathrm{M}_{0}}-\left(h_{1}\right) z_{\mathrm{M}_{1}}+\left(h_{1} g_{1}\right) z_{\mathrm{M}_{1}}-\left(g_{1}\right) z_{\mathrm{M}_{1}},
\end{aligned}
$$

that is, $\left(h_{1} g_{1}\right) z_{\mathrm{M}_{1}}=\left(h_{1}\right) z_{\mathrm{M}_{1}}+\left(g_{1}\right) z_{\mathrm{M}_{1}}-\left(h_{1} \partial, g_{1} \partial\right) z_{\mathrm{M}_{0}}$ for $g_{1}, h_{1} \in \mathrm{M}_{1} G$.

(iv) We have, using (i),

$$
\begin{aligned}
0= & \left(1,1,1, g_{0}, g_{1}, 1,1\right)(z \partial) \\
= & \left(1, g_{0}, g_{1} \partial\right) z-\left(1, g_{0}, 1\right) z+\left({ }^{g_{0} \mathrm{~s}_{0}} g_{1}, g_{0}, 1\right) z-g_{0} \mathrm{~B}_{0} \mathrm{M} G \cdot\left(g_{1}, 1,1\right) z \\
= & \left(g_{0}, g_{1} \partial\right) z_{\mathrm{M}_{0}}+\left({ }^{g_{0} \mathrm{~s}_{0}} g_{1}\right) z_{\mathrm{M}_{1}}-\left({ }^{g_{0}}\left(g_{1} \partial\right), g_{0}\right) z_{\mathrm{M}_{0}} \\
& \quad-g_{0} \mathrm{~B}_{0} \mathrm{M} G \cdot\left(g_{1}\right) z_{\mathrm{M}_{1}},
\end{aligned}
$$

that is, $\left({ }^{g_{0} \mathrm{~s}_{0}} g_{1}\right) z_{\mathrm{M}_{1}}=g_{0} \mathrm{~B}_{0} \mathrm{M} G \cdot\left(g_{1}\right) z_{\mathrm{M}_{1}}+\left({ }^{g_{0}}\left(g_{1} \partial\right), g_{0}\right) z_{\mathrm{M}_{0}}-\left(g_{0}, g_{1} \partial\right) z_{\mathrm{M}_{0}}$ for $g_{1} \in \mathrm{M}_{1} G, g_{0} \in \mathrm{M}_{0} G$.

(v) We have

$$
\begin{aligned}
0 & =\left(g_{2}, 1,1,1,1,1,1\right)(z \partial) \\
& =\left(g_{2} \partial, 1,1\right) z-(1,1,1) z+(1,1,1) z-(1,1,1) z \\
& =\left(g_{2} \partial\right) z_{\mathrm{M}_{1}}-(1) z_{\mathrm{M}_{1}},
\end{aligned}
$$

that is, $\left(g_{2} \partial\right) z_{\mathrm{M}_{1}}=(1) z_{\mathrm{M}_{1}}$ for $g_{2} \in \mathrm{M}_{2} G$.

Now let us conversely suppose given an analysed 2-cochain $z \in \mathrm{Ch}_{\text {an }}^{2}(G, M)$ that 
fulfills the properties (i) to (v). Then we compute

$$
\begin{aligned}
& \left(g_{2}, k_{1}, h_{1}, g_{1}, k_{0}, h_{0}, g_{0}\right)(z \partial) \\
& =\left(\left(g_{2} \partial\right) k_{1},\left(h_{1} \partial\right) k_{0},\left(g_{1} \partial\right) h_{0}\right) z-\left(k_{1} h_{1}, k_{0}, h_{0} g_{0}\right) z+\left(h_{1}{ }^{k_{0} \mathrm{~s}_{0}} g_{1}, k_{0} h_{0}, g_{0}\right) z \\
& -k_{0} \mathrm{~B}_{0} \mathrm{M} G \cdot\left(g_{1}, h_{0}, g_{0}\right) z \\
& =\left(\left(g_{2} \partial\right) k_{1}\right) z_{\mathrm{M}_{1}}-\left(k_{1} \partial,\left(h_{1} \partial\right) k_{0}\right) z_{\mathrm{M}_{0}}+\left(\left(h_{1} \partial\right) k_{0},\left(g_{1} \partial\right) h_{0}\right) z_{\mathrm{M}_{0}}-\left(k_{1} h_{1}\right) z_{\mathrm{M}_{1}} \\
& +\left(\left(k_{1} h_{1}\right) \partial, k_{0}\right) z_{\mathrm{M}_{0}}-\left(k_{0}, h_{0} g_{0}\right) z_{\mathrm{M}_{0}}+\left(h_{1}{ }^{k_{0} \mathrm{~s}_{0}} g_{1}\right) z_{\mathrm{M}_{1}} \\
& -\left(\left(h_{1}{ }^{k_{0} \mathrm{~s}_{0}} g_{1}\right) \partial, k_{0} h_{0}\right) z_{\mathrm{M}_{0}}+\left(k_{0} h_{0}, g_{0}\right) z_{\mathrm{M}_{0}}-k_{0} \mathrm{~B}_{0} \mathrm{M} G \cdot\left(g_{1}\right) z_{\mathrm{M}_{1}} \\
& +k_{0} \mathrm{~B}_{0} \mathrm{M} G \cdot\left(g_{1} \partial, h_{0}\right) z_{\mathrm{M}_{0}}-k_{0} \mathrm{~B}_{0} \mathrm{M} G \cdot\left(h_{0}, g_{0}\right) z_{\mathrm{M}_{0}} \\
& =\left(\left(g_{2} \partial\right) k_{1}\right) z_{\mathrm{M}_{1}}-\left(k_{1} h_{1}\right) z_{\mathrm{M}_{1}}+\left(h_{1}{ }^{k_{0} \mathrm{~s}_{0}} g_{1}\right) z_{\mathrm{M}_{1}}-k_{0} \mathrm{~B}_{0} \mathrm{M} G \cdot\left(g_{1}\right) z_{\mathrm{M}_{1}} \\
& -\left(k_{1} \partial,\left(h_{1} \partial\right) k_{0}\right) z_{\mathrm{M}_{0}}+\left(\left(h_{1} \partial\right) k_{0},\left(g_{1} \partial\right) h_{0}\right) z_{\mathrm{M}_{0}}+\left(\left(k_{1} \partial\right)\left(h_{1} \partial\right), k_{0}\right) z_{\mathrm{M}_{0}} \\
& -\left(\left(h_{1} \partial\right)^{k_{0}}\left(g_{1} \partial\right), k_{0} h_{0}\right) z_{\mathrm{M}_{0}}+k_{0} \mathrm{~B}_{0} \mathrm{MG} \cdot\left(g_{1} \partial, h_{0}\right) z_{\mathrm{M}_{0}}-\left(k_{0}, h_{0} g_{0}\right) z_{\mathrm{M}_{0}} \\
& +\left(k_{0} h_{0}, g_{0}\right) z_{\mathrm{M}_{0}}-k_{0} \mathrm{~B}_{0} \mathrm{MG} \cdot\left(h_{0}, g_{0}\right) z_{\mathrm{M}_{0}} \\
& =\left(g_{2} \partial\right) z_{\mathrm{M}_{1}}+\left(k_{1}\right) z_{\mathrm{M}_{1}}-\left(1, k_{1} \partial\right) z_{\mathrm{M}_{0}}-\left(k_{1} h_{1}\right) z_{\mathrm{M}_{1}}+\left(h_{1}\right) z_{\mathrm{M}_{1}}+\left({ }^{k_{0} \mathrm{~s}_{0}} g_{1}\right) z_{\mathrm{M}_{1}} \\
& -\left(h_{1} \partial,\left({ }^{k_{0} \mathrm{~s}_{0}} g_{1}\right) \partial\right) z_{\mathrm{M}_{0}}-\left({ }^{k_{0} \mathrm{~s}_{0}} g_{1}\right) z_{\mathrm{M}_{1}}+\left(\left({ }^{k_{0} \mathrm{~s}_{0}} g_{1}\right) \partial, k_{0}\right) z_{\mathrm{M}_{0}} \\
& -\left(k_{0}, g_{1} \partial\right) z_{\mathrm{M}_{0}}-\left(k_{1} \partial,\left(h_{1} \partial\right) k_{0}\right) z_{\mathrm{M}_{0}}+\left(\left(h_{1} \partial\right) k_{0},\left(g_{1} \partial\right) h_{0}\right) z_{\mathrm{M}_{0}} \\
& +\left(\left(k_{1} \partial\right)\left(h_{1} \partial\right), k_{0}\right) z_{\mathrm{M}_{0}}-\left(\left(h_{1} \partial\right)^{k_{0}}\left(g_{1} \partial\right), k_{0} h_{0}\right) z_{\mathrm{M}_{0}} \\
& +k_{0} \mathrm{~B}_{0} \mathrm{M} G \cdot\left(g_{1} \partial, h_{0}\right) z_{\mathrm{M}_{0}}-\left(k_{0}, h_{0}\right) z_{\mathrm{M}_{0}} \\
& =\left(k_{1}\right) z_{\mathrm{M}_{1}}-\left(k_{1} h_{1}\right) z_{\mathrm{M}_{1}}+\left(h_{1}\right) z_{\mathrm{M}_{1}}-\left(h_{1} \partial,{ }^{k_{0}}\left(g_{1} \partial\right)\right) z_{\mathrm{M}_{0}}+\left({ }^{k_{0}}\left(g_{1} \partial\right), k_{0}\right) z_{\mathrm{M}_{0}} \\
& -\left(k_{0}, g_{1} \partial\right) z_{\mathrm{M}_{0}}-\left(k_{1} \partial,\left(h_{1} \partial\right) k_{0}\right) z_{\mathrm{M}_{0}}+\left(\left(h_{1} \partial\right) k_{0},\left(g_{1} \partial\right) h_{0}\right) z_{\mathrm{M}_{0}} \\
& +\left(\left(k_{1} \partial\right)\left(h_{1} \partial\right), k_{0}\right) z_{\mathrm{M}_{0}}-\left(\left(h_{1} \partial\right)^{k_{0}}\left(g_{1} \partial\right), k_{0} h_{0}\right) z_{\mathrm{M}_{0}} \\
& +k_{0} \mathrm{~B}_{0} \mathrm{M} G \cdot\left(g_{1} \partial, h_{0}\right) z_{\mathrm{M}_{0}}-\left(k_{0}, h_{0}\right) z_{\mathrm{M}_{0}} \\
& =\left(k_{1} \partial, h_{1} \partial\right) z_{\mathrm{M}_{0}}-\left(k_{1} \partial,\left(h_{1} \partial\right) k_{0}\right) z_{\mathrm{M}_{0}}+\left(\left(k_{1} \partial\right)\left(h_{1} \partial\right), k_{0}\right) z_{\mathrm{M}_{0}} \\
& -\left(h_{1} \partial,{ }^{k_{0}}\left(g_{1} \partial\right)\right) z_{\mathrm{M}_{0}}-\left(\left(h_{1} \partial\right)^{k_{0}}\left(g_{1} \partial\right), k_{0} h_{0}\right) z_{\mathrm{M}_{0}}+\left({ }^{k_{0}}\left(g_{1} \partial\right), k_{0}\right) z_{\mathrm{M}_{0}} \\
& -\left(k_{0}, h_{0}\right) z_{\mathrm{M}_{0}}-\left(k_{0}, g_{1} \partial\right) z_{\mathrm{M}_{0}}+k_{0} \mathrm{~B}_{0} \mathrm{M} G \cdot\left(g_{1} \partial, h_{0}\right) z_{\mathrm{M}_{0}} \\
& +\left(\left(h_{1} \partial\right) k_{0},\left(g_{1} \partial\right) h_{0}\right) z_{\mathrm{M}_{0}} \\
& =\left(h_{1} \partial, k_{0}\right) z_{\mathrm{M}_{0}}-\left({ }^{k_{0}}\left(g_{1} \partial\right), k_{0} h_{0}\right) z_{\mathrm{M}_{0}}-\left(h_{1} \partial,{ }^{k_{0}}\left(g_{1} \partial\right) k_{0} h_{0}\right) z_{\mathrm{M}_{0}} \\
& +\left({ }^{k_{0}}\left(g_{1} \partial\right), k_{0} h_{0}\right) z_{\mathrm{M}_{0}}-\left({ }^{k_{0}}\left(g_{1} \partial\right) k_{0}, h_{0}\right) z_{\mathrm{M}_{0}}-\left(k_{0},\left(g_{1} \partial\right) h_{0}\right) z_{\mathrm{M}_{0}} \\
& +\left(k_{0}\left(g_{1} \partial\right), h_{0}\right) z_{\mathrm{M}_{0}}+\left(\left(h_{1} \partial\right) k_{0},\left(g_{1} \partial\right) h_{0}\right) z_{\mathrm{M}_{0}} \\
& =\left(h_{1} \partial, k_{0}\right) z_{\mathrm{M}_{0}}+\left(\left(h_{1} \partial\right) k_{0},\left(g_{1} \partial\right) h_{0}\right) z_{\mathrm{M}_{0}}-\left(h_{1} \partial, k_{0}\left(g_{1} \partial\right) h_{0}\right) z_{\mathrm{M}_{0}} \\
& -\left(k_{0},\left(g_{1} \partial\right) h_{0}\right) z_{\mathrm{M}_{0}} \\
& =0
\end{aligned}
$$

for all $g_{0}, h_{0}, k_{0} \in \mathrm{M}_{0} G, g_{1}, h_{1}, k_{1} \in \mathrm{M}_{1} G, g_{2} \in \mathrm{M}_{2} G$, that is, $z \in \mathrm{Z}_{\text {an }}^{2}(G, M)$.

(b) This follows from (a) by definition of the 2-cocycles of $V$ via $\operatorname{Cosk}_{1} V$ and the fact that $\mathrm{M}_{0} \operatorname{Cosk}_{1} V=\operatorname{Gp} V, \mathrm{M}_{1} \operatorname{Cosk}_{1} V=\mathrm{Mp} V$ and $\mathrm{M}_{2} \operatorname{Cosk}_{1} V=\{1\}$ (up to simplified notation).

With the preceeding proposition we can now establish a description of the second 
analysed cocycle group of a simplicial group resp. of a crossed module as a pullback. This can be seen as a continuation of proposition 3.4.

\section{Corollary 3.12.}

(a) Given a simplicial group $G$ and an abelian $\pi_{0}(G)$-module $M$, the diagram

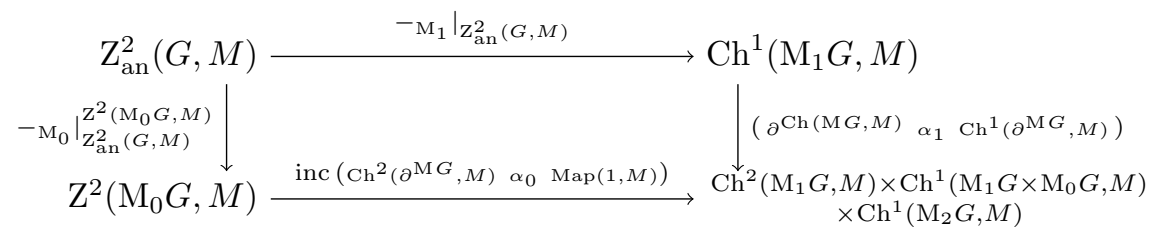

is a pullback of abelian groups, where $\left(g_{1}, g_{0}\right)\left(c_{1} \alpha_{1}\right):=\left({ }^{g_{0} \mathrm{~s}_{0}} g_{1}\right) c_{1}-g_{0} \mathrm{~B}_{0} \mathrm{M} G$. $\left(g_{1}\right) c_{1} \quad$ and $\left(g_{1}, g_{0}\right)\left(c_{0} \alpha_{0}\right):=\left({ }^{g_{0}}\left(g_{1} \partial\right), g_{0}\right) c_{0}-\left(g_{0}, g_{1} \partial\right) c_{0} \quad$ for $\quad g_{1} \in \mathrm{M}_{1} G$, $g_{0} \in \mathrm{M}_{0} G, c_{1} \in \mathrm{Ch}^{1}\left(\mathrm{M}_{1} G, M\right), c_{0} \in \mathrm{Ch}^{2}\left(\mathrm{M}_{0} G, M\right)$, and where $M$ is considered as a trivial $\mathrm{M}_{1} G$-module.

(b) Given a crossed module $V$ and an abelian $\pi_{0}(V)$-module $M$, the diagram

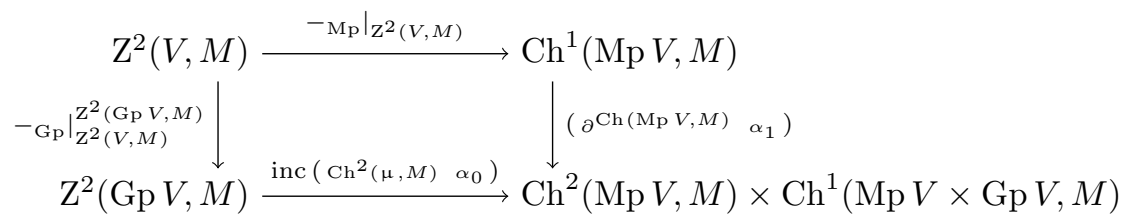

is a pullback of abelian groups, where $(m, g)\left(c_{1} \alpha_{1}\right):=\left({ }^{g} m\right) c_{1}-g(\operatorname{Im} \mu) \cdot(m) c_{1}$ and $(m, g)\left(c_{0} \alpha_{0}\right):=\left({ }^{g} m, g\right) c_{0}-(g, m) c_{0} \quad$ for $\quad m \in \operatorname{Mp} V, \quad g \in \operatorname{Gp} V$, $c_{1} \in \mathrm{Ch}^{1}(\mathrm{Mp} V, M), c_{0} \in \mathrm{Ch}^{2}(\mathrm{Gp} V, M)$, and where $M$ is considered as a trivial $\mathrm{Mp} V$-module. In particular, we have an isomorphism

$$
\begin{aligned}
\mathrm{Z}^{2}(V, M) \rightarrow & \left\{\left(c_{1}, z_{0}\right) \in \mathrm{Ch}^{1}(\mathrm{Mp} V, M) \times \mathrm{Z}^{2}(\mathrm{Gp} V, M) \mid\right. \\
& (n m) c_{1}=n c_{1}+m c_{1}-(n, m) z_{0} \text { and } \\
& \left({ }^{g} m\right) c_{1}=g(\operatorname{Im} \mu) \cdot(m) c_{1}+\left({ }^{g} m, g\right) z_{0}-(g, m) z_{0} \\
& \text { for all } m, n \in \mathrm{Mp} V, g \in \mathrm{Gp} V\}, \\
z \mapsto & \left(z_{\mathrm{M}_{1}}, z_{\mathrm{M}_{0}}\right) .
\end{aligned}
$$

Proof.

(a) We note that $\alpha_{0}$ and $\alpha_{1}$ are group homomorphisms. By proposition 3.11(a)(ii) to (v), the diagram is well-defined and commutes. To show that it is a pullback, we suppose given an arbitrary abelian group $T$ and group homomorphisms $\varphi_{0}: T \rightarrow \mathrm{Z}^{2}\left(\mathrm{M}_{0} G, M\right)$ and $\varphi_{1}: T \rightarrow \mathrm{Ch}^{1}\left(\mathrm{M}_{1} G, M\right)$ with $\varphi_{0}$ inc $\mathrm{Ch}^{2}\left(\partial^{\mathrm{M} G}, M\right)=$ $\varphi_{1} \partial^{\mathrm{Ch}(\mathrm{M} G, M)}, \quad \varphi_{0}$ inc $\alpha_{0}=\varphi_{1} \alpha_{1} \quad$ and $\varphi_{0} \operatorname{inc} \operatorname{Map}(1, M)=\varphi_{1} \mathrm{Ch}^{1}\left(\partial^{\mathrm{M} G}, M\right)$. For every $t \in T$, we define a 2 -cochain $t \varphi \in \mathrm{Ch}_{\text {an }}^{2}(G, M)$ by $\left(g_{1}, h_{0}, g_{0}\right)(t \varphi):=$ $\left(g_{1}\right)\left(t \varphi_{1}\right)-\left(g_{1} \partial, h_{0}\right)\left(t \varphi_{0}\right)+\left(h_{0}, g_{0}\right)\left(t \varphi_{0}\right)$ for $g_{1} \in \mathrm{M}_{1} G, g_{0}, h_{0} \in \mathrm{M}_{0} G$. Since

$$
\begin{aligned}
\left(g_{1}\right)(t \varphi)_{\mathrm{M}_{1}} & =\left(g_{1}, 1,1\right)(t \varphi)=\left(g_{1}\right)\left(t \varphi_{1}\right)-\left(g_{1} \partial, 1\right)\left(t \varphi_{0}\right)+(1,1)\left(t \varphi_{0}\right) \\
& =\left(g_{1}\right)\left(t \varphi_{1}\right)
\end{aligned}
$$


for all $g_{1} \in \mathrm{M}_{1} G$ and

$$
\begin{aligned}
\left(h_{0}, g_{0}\right)(t \varphi)_{\mathrm{M}_{0}} & =\left(1, h_{0}, g_{0}\right)(t \varphi)=(1)\left(t \varphi_{1}\right)-\left(1, h_{0}\right)\left(t \varphi_{0}\right)+\left(h_{0}, g_{0}\right)\left(t \varphi_{0}\right) \\
& =\left(h_{0}, g_{0}\right)\left(t \varphi_{0}\right)
\end{aligned}
$$

for all $g_{0}, h_{0} \in \mathrm{M}_{0} G$, it follows that $(t \varphi)_{\mathrm{M}_{1}}=t \varphi_{1}$ and $(t \varphi)_{\mathrm{M}_{0}}=t \varphi_{0}$ and hence $t \varphi \in \mathrm{Z}_{\text {an }}^{2}(G, M)$ for all $t \in T$ by proposition 3.11(a). Thus we obtain a well-defined group homomorphism $\varphi: T \rightarrow \mathrm{Z}_{\text {an }}^{2}(G, M)$ with $(t \varphi)_{\mathrm{M}_{1}}=t \varphi_{1}$ and $(t \varphi)_{\mathrm{M}_{0}}=t \varphi_{0}$ for all $t \in T$. The uniqueness of such a map follows from 3.11(a)(i).

Now we are able to show that the second cohomology group of a simplicial group only depends on its 1-segment.

Proposition 3.13. Given a simplicial group $G$ and an abelian $\pi_{0}(G)$-module $M$, the unit component $\varepsilon_{G}: G \rightarrow \operatorname{Cosk}_{1} \operatorname{Trunc}^{1} G$ of the adjunction Trunc $^{1} \dashv$ Cosk $_{1}$ induces an isomorphism

$$
\mathrm{Z}_{\text {an }}^{2}\left(\varepsilon_{G}, M\right): \mathrm{Z}^{2}\left(\operatorname{Trunc}^{1} G, M\right) \rightarrow \mathrm{Z}_{\text {an }}^{2}(G, M),
$$

which in turn induces isomorphisms $\mathrm{B}^{2}\left(\varepsilon_{G}, M\right)$ and $\mathrm{H}^{2}\left(\varepsilon_{G}, M\right)$. In particular, we have

$$
\mathrm{H}^{2}(G, M) \cong \mathrm{H}^{2}\left(\operatorname{Trunc}^{1} G, M\right) .
$$

Proof. For $n \in \mathbb{N}_{0}$, we denote by $\varphi_{n}$ the isomorphisms from $G_{n}$ to its semidirect product decomposition, cf. section 2.7. Then we have $\left(g_{0}\right) \varphi_{0}^{-1}\left(\varepsilon_{G}\right)_{0}=\left(g_{0}\right)$ and $\left(g_{1}, h_{0}\right) \varphi_{1}^{-1}\left(\varepsilon_{G}\right)_{1}=\left(g_{1} \pi, h_{0}\right)$ for $g_{1} \in \mathrm{M}_{1} G, g_{0}, h_{0} \in \mathrm{M}_{0} G$, where we let $\pi: \mathrm{M}_{1} G \rightarrow$ $\mathrm{M}_{1} G / \mathrm{B}_{1} \mathrm{M} G=\mathrm{Mp} \operatorname{Trunc}^{1} G$ denote the canonical epimorphism, cf. section 2.5. Therefore the group homomorphism $\mathrm{Z}_{\text {an }}^{2}\left(\varepsilon_{G}, M\right)$ is given by $\left(g_{1}, h_{0}, g_{0}\right)\left(z^{\prime} \mathrm{Z}_{\text {an }}^{2}\left(\varepsilon_{G}, M\right)\right)$ $=\left(g_{1} \pi, h_{0}, g_{0}\right) z^{\prime}$ for $g_{1} \in \mathrm{M}_{1} G, g_{0}, h_{0} \in \mathrm{M}_{0} G, z^{\prime} \in \mathrm{Z}^{2}\left(\operatorname{Trunc}^{1} G, M\right)$. Thus we have $z^{\prime} \mathrm{Z}_{\text {an }}^{2}\left(\varepsilon_{G}, M\right)=0$ if and only if already $z^{\prime}=0$, that is, $\mathrm{Z}_{\text {an }}^{2}\left(\varepsilon_{G}, M\right)$ is injective.

To show surjectivity, we suppose given an analysed 2-cochain $z \in \mathrm{Z}_{\mathrm{an}}^{2}(G, M)$. We choose a section of the underlying pointed map of $\pi$, that is, a pointed map $s$ : $\operatorname{Mp} \operatorname{Trunc}^{1} G \rightarrow \mathrm{M}_{1} G$ with $s \pi=\operatorname{id}_{\mathrm{Mp}_{\operatorname{Trunc}}{ }^{1}}$. Then $(n s)(m s)((n m) s)^{-1} \in \operatorname{Ker} \pi=$ $\mathrm{B}_{1} \mathrm{M} G$ and therefore

$$
((n s)(m s)) z_{\mathrm{M}_{1}}=\left((n s)(m s)((n m) s)^{-1}((n m) s)\right) z_{\mathrm{M}_{1}}=((n m) s) z_{\mathrm{M}_{1}}
$$

for all $m, n \in \operatorname{Mp}_{\operatorname{Trunc}}{ }^{1} G$. Moreover, $\left(\left({ }^{g} m\right) s\right)\left({ }^{g s_{0}}(m s)\right)^{-1} \in \operatorname{Ker} \pi=\mathrm{B}_{1} \mathrm{M} G$ implies

$$
\left(\left({ }^{g} m\right) s\right) z_{\mathrm{M}_{1}}=\left(\left(\left({ }^{g} m\right) s\right)\left(^{g \mathrm{~s}_{0}}(m s)\right)^{-1}{ }^{g \mathrm{~s}_{0}}(m s)\right) z_{\mathrm{M}_{1}}=\left({ }^{g \mathrm{~s}_{0}}(m s)\right) z_{\mathrm{M}_{1}}
$$

for all $m \in \operatorname{Mp} \operatorname{Trunc}^{1} G, g \in \operatorname{Gp}_{\operatorname{Trunc}}{ }^{1} G$. Defining $c_{1}^{\prime}: \operatorname{Mp}_{\operatorname{Trunc}}{ }^{1} G \rightarrow M$ by $(m) c_{1}^{\prime}$ $:=(m s) z_{\mathrm{M}_{1}}$ for $m \in \operatorname{Mp~Trunc~}^{1} G$, we obtain

$$
\begin{aligned}
(n m) c_{1}^{\prime} & =((n m) s) z_{\mathrm{M}_{1}}=((n s)(m s)) z_{\mathrm{M}_{1}}=(n s) z_{\mathrm{M}_{1}}+(m s) z_{\mathrm{M}_{1}}-(n s \partial, m s \partial) \\
& =(n) c_{1}^{\prime}+(m) c_{1}^{\prime}-(n, m) z_{\mathrm{M}_{0}}
\end{aligned}
$$

for all $m, n \in \operatorname{Mp~Trunc}^{1} G$ as well as

$$
\begin{aligned}
\left({ }^{g} m\right) c_{1}^{\prime} & =\left(\left({ }^{g} m\right) s\right) z_{\mathrm{M}_{1}}=\left({ }^{g s_{0}}(m s)\right) z_{\mathrm{M}_{1}} \\
& =g \mathrm{~B}_{0} \mathrm{M} G \cdot(m s) z_{\mathrm{M}_{1}}+\left({ }^{g}(m s \partial), g\right) z_{\mathrm{M}_{0}}-(g, m s \partial) z_{\mathrm{M}_{0}} \\
& =g(\operatorname{Im} \mu) \cdot(m) c_{1}^{\prime}+\left({ }^{g} m, g\right) z_{\mathrm{M}_{0}}-(g, m) z_{\mathrm{M}_{0}}
\end{aligned}
$$


for all $m \in \operatorname{Mp} \operatorname{Trunc}^{1} G, g \in \operatorname{Gp}_{\operatorname{Trunc}}{ }^{1} G$. Thus we get a well-defined 2-cocycle $z^{\prime} \in$ $\mathrm{Z}^{2}\left(\operatorname{Trunc}^{1} G, M\right)$ with $(m) z_{\mathrm{Mp}}^{\prime}=(m s) z_{\mathrm{M}_{1}}$ for $m \in \operatorname{MpTrunc}^{1} G$ and $z_{\mathrm{Gp}}^{\prime}=z_{\mathrm{M}_{0}}$ by corollary 3.12(b). Further, $g_{1}\left(g_{1} \pi s\right)^{-1} \in \operatorname{Ker} \pi=\mathrm{B}_{1} \mathrm{M} G$ implies

$$
\begin{aligned}
0 & =\left(g_{1}\left(g_{1} \pi s\right)^{-1}\right) z_{\mathrm{M}_{1}}-(1) z_{\mathrm{M}_{1}} \\
& =\left(g_{1}\right) z_{\mathrm{M}_{1}}+\left(\left(g_{1} \pi s\right)^{-1}\right) z_{\mathrm{M}_{1}}-\left(g_{1} \partial,\left(g_{1} \pi s\right)^{-1} \partial\right) z_{\mathrm{M}_{0}}-(1) z_{\mathrm{M}_{1}} \\
& =\left(g_{1}\right) z_{\mathrm{M}_{1}}+\left(\left(g_{1} \pi s\right)^{-1}\right) z_{\mathrm{M}_{1}}-\left(g_{1} \pi s \partial,\left(g_{1} \pi s\right)^{-1} \partial\right) z_{\mathrm{M}_{0}}-\left(\left(g_{1} \pi s\right)\left(g_{1} \pi s\right)^{-1}\right) z_{\mathrm{M}_{1}} \\
& =\left(g_{1}\right) z_{\mathrm{M}_{1}}-\left(g_{1} \pi s\right) z_{\mathrm{M}_{1}}
\end{aligned}
$$

for all $g_{1} \in \mathrm{M}_{1} G$. But now it follows that $z^{\prime} \mathrm{Z}_{\text {an }}^{2}\left(\varepsilon_{G}, M\right)=z$ since

$$
\begin{aligned}
\left(g_{1}, h_{0}, g_{0}\right)\left(z^{\prime} \mathrm{Z}_{\mathrm{an}}^{2}\left(\varepsilon_{G}, M\right)\right) & =\left(g_{1} \pi, h_{0}, g_{0}\right) z^{\prime} \\
& =\left(g_{1} \pi\right) z_{\mathrm{Mp}}^{\prime}-\left(g_{1} \pi, h_{0}\right) z_{\mathrm{Gp}}^{\prime}+\left(h_{0}, g_{0}\right) z_{\mathrm{Gp}}^{\prime} \\
& =\left(g_{1} \pi s\right) z_{\mathrm{M}_{1}}-\left(g_{1} \partial, h_{0}\right) z_{\mathrm{M}_{0}}+\left(h_{0}, g_{0}\right) z_{\mathrm{M}_{0}} \\
& =\left(g_{1}\right) z_{\mathrm{M}_{1}}-\left(g_{1} \partial, h_{0}\right) z_{\mathrm{M}_{0}}+\left(h_{0}, g_{0}\right) z_{\mathrm{M}_{0}} \\
& =\left(g_{1}, h_{0}, g_{0}\right) z
\end{aligned}
$$

for all $g_{1} \in \mathrm{M}_{1} G, g_{0}, h_{0} \in \mathrm{M}_{0} G$. Thus $\mathrm{Z}_{\text {an }}^{2}\left(\varepsilon_{G}, M\right)$ is surjective. Altogether, the induced group homomorphism $\mathrm{Z}_{\mathrm{an}}^{2}\left(\varepsilon_{G}, M\right)$ is bijective and hence an isomorphism of abelian groups.

The injectivity of $\mathrm{Z}_{\mathrm{an}}^{2}\left(\varepsilon_{G}, M\right)$ implies the injectivity of the restriction $\mathrm{B}_{\mathrm{an}}^{2}\left(\varepsilon_{G}, M\right)$. To show that this is also an isomorphism, it remains to show that for a given analysed 2-coboundary $b \in \mathrm{B}_{\text {an }}^{2}(G, M)$, the 2-cocycle $b^{\prime} \in \mathrm{Z}^{2}\left(\operatorname{Trunc}^{1} G, M\right)$ given by $(m) b_{\mathrm{Mp}}^{\prime}=(m s) b_{\mathrm{M}_{1}}$ for $m \in \mathrm{Mp} \operatorname{Trunc}^{1} G$ and $b_{\mathrm{Gp}}^{\prime}=b_{\mathrm{M}_{0}}$ is in fact a 2-coboundary in $\mathrm{B}^{2}\left(\operatorname{Trunc}^{1} G, M\right)$.

We choose $c \in \mathrm{Ch}_{\mathrm{an}}^{1}(G, M)=\mathrm{Ch}^{1}\left(\operatorname{Trunc}^{1} G, M\right)$ with $b=c \partial^{\mathrm{Ch}_{\text {an }}(G, M)}$, that is, with $\left(g_{1}, h_{0}, g_{0}\right) b=\left(\left(g_{1} \partial\right) h_{0}\right) c-\left(h_{0} g_{0}\right) c+h_{0} \mathrm{~B}_{0} \mathrm{M} G \cdot\left(g_{0}\right) c$ for $g_{1} \in \mathrm{M}_{1} G, g_{0}, h_{0} \in$ $\mathrm{M}_{0} G$. It follows that

$$
(m) b_{\mathrm{Mp}}^{\prime}=(m s) b_{\mathrm{M}_{1}}=(m s \partial) c=(m) c=(m)\left(c \partial^{\operatorname{Ch}\left(\operatorname{Trunc}^{1} G, M\right)}\right)_{\mathrm{Mp}}
$$

for all $m \in \operatorname{Mp}_{\operatorname{Trunc}}^{1} G$, that is, $b_{\mathrm{Mp}}^{\prime}=\left(c \partial^{\operatorname{Ch}\left(\operatorname{Trunc}^{1} G, M\right)}\right)_{\mathrm{Mp}}$, as well as

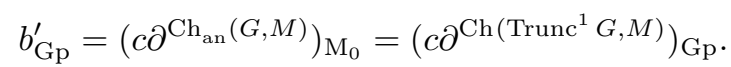

Hence we have $b^{\prime}=c \partial^{\mathrm{Ch}\left(\operatorname{Trunc}^{1} G, M\right)} \in \mathrm{B}^{2}\left(\operatorname{Trunc}^{1} G, M\right)$.

We have shown that $\mathrm{Z}_{\mathrm{an}}^{2}\left(\varepsilon_{G}, M\right)$ and $\mathrm{B}_{\mathrm{an}}^{2}\left(\varepsilon_{G}, M\right)$ are isomorphisms, and hence $\mathrm{H}_{\mathrm{an}}^{2}\left(\varepsilon_{G}, M\right)$ is also an isomorphism. In particular, we have

$$
\mathrm{H}^{2}(G, M) \cong \mathrm{H}_{\mathrm{an}}^{2}(G, M) \cong \mathrm{H}^{2}\left(\operatorname{Trunc}^{1} G, M\right)
$$

\section{Crossed module extensions and standard 2-cocycles}

Throughout this section, we suppose given a group $\Pi_{0}$ and abelian $\Pi_{0}$-modules $\Pi_{1}$ and $M$, where $\Pi_{1}$ is written multiplicatively. Moreover, we suppose given a crossed module extension $E$ of $\Pi_{0}$ with $\Pi_{1}$ and a section system $\left(s^{1}, s^{0}\right)$ for $E$. The lifting system coming from $\left(s^{1}, s^{0}\right)$ will be denoted by $\left(Z^{2}, Z^{1}\right)$, that is, $Z^{1}=s^{0}$ and $Z^{2}=$ $\mathrm{z}^{2} s^{1}$. Cf. section 2.13 . 
Notation 4.1. In this section, we use the following conventions and notations: For $p, q, r \in \Pi_{0}$, we write $[p]:=p Z^{1},[q, p]:=(q, p) Z^{2}$ and $[r, q, p]:=(r, q, p) \mathrm{z}^{3}$. For $g \in$ $\operatorname{Im} \mu$, we write $[g]:=g s^{1}$. So for $m \in \operatorname{Mp} E$, we usually write $[m]=[m \mu]=m \mu s^{1}$, following our convention from section 2.4. Finally, for $g \in \operatorname{Gp} E$, we write $\bar{g}:=g \pi$.

With these conventions, we have $\overline{[p]}=p$ and $[q, p]=\left[[q][p][q p]^{-1}\right]$ and $[r, q, p] \iota=$ $[r, q][r q, p][r, q p]^{-1[r]}\left([q, p]^{-1}\right)$ for $p, q, r \in \Pi_{0}$ and $[m] \mu=m \mu$ for $m \in \operatorname{Mp} E$.

We have seen in section 2.12, how the computation of cohomology groups in positive dimension can be reduced to that of pointed cohomology groups. In this section, we will see a further reduction in the case where we consider the second cohomology group of the underlying crossed module of a crossed module extension.

Definition 4.2 (standardisation of pointed 2-cocycles).

(a) Given a pointed 2-cocycle $z \in \mathrm{Z}_{\mathrm{pt}}^{2}(E, M)$, the standardisation of $z$ (with respect to $\left.\left(s^{1}, s^{0}\right)\right)$ is given by

$$
z^{\mathrm{st}}=z^{\mathrm{st},\left(s^{1}, s^{0}\right)}:=z-\mathrm{s}_{z} \partial,
$$

where the standardiser of $z$ (with respect to $\left(s^{1}, s^{0}\right)$ ) is defined to be the pointed 1-cochain $\mathrm{s}_{z}=\mathrm{s}_{z}^{\left(s^{1}, s^{0}\right)} \in \mathrm{Ch}_{\mathrm{pt}}^{1}(E, M)$ given by

$$
(g) \mathrm{s}_{z}:=\left(\left[g[\bar{g}]^{-1}\right],[\bar{g}], 1\right) z
$$

for $g \in \mathrm{Gp} E$.

(b) A pointed 2-cocycle $z \in \mathrm{Z}_{\mathrm{pt}}^{2}(E, M)$ is said to be standard (with respect to $\left(s^{1}, s^{0}\right)$ ) (or a standard 2-cocycle, for short) if $z^{\text {st }}=z$. The subgroup of $\mathrm{Z}_{\mathrm{pt}}^{2}(E, M)$ consisting of all standard 2-cocycles of $E$ with coefficients in $M$ will be denoted by

$$
\mathrm{Z}_{\mathrm{st}}^{2}(E, M)=\mathrm{Z}_{\mathrm{st},\left(s^{1}, s^{0}\right)}^{2}(E, M):=\left\{z \in \mathrm{Z}_{\mathrm{pt}}^{2}(E, M) \mid z^{\mathrm{st}}=z\right\} .
$$

Likewise, the subgroup of $\mathrm{B}_{\mathrm{pt}}^{2}(E, M)$ consisting of all standard 2-coboundaries of $E$ with coefficients in $M$ will be denoted by

$$
\mathrm{B}_{\mathrm{st}}^{2}(E, M)=\mathrm{B}_{\mathrm{st},\left(s^{1}, s^{0}\right)}^{2}(E, M):=\left\{b \in \mathrm{B}_{\mathrm{pt}}^{2}(E, M) \mid b^{\mathrm{st}}=b\right\} .
$$

Moreover, we set

$$
\mathrm{H}_{\mathrm{st}}^{2}(E, M)=\mathrm{H}_{\mathrm{st},\left(s^{1}, s^{0}\right)}^{2}(E, M):=\mathrm{Z}_{\mathrm{st}}^{2}(E, M) / \mathrm{B}_{\mathrm{st}}^{2}(E, M) .
$$

Remark 4.3. We have

$$
(g) \mathrm{s}_{z}=\left(\left[g[\bar{g}]^{-1}\right]\right) z_{\mathrm{Mp}}-\left(g[\bar{g}]^{-1},[\bar{g}]\right) z_{\mathrm{Gp}}
$$

for $g \in \mathrm{Gp} E, z \in \mathrm{Z}_{\mathrm{pt}}^{2}(E, M)$.

Proof. This follows from proposition 3.11(b)(i).

In the next proposition, we give more detailed formulas for the standardisation. 


\section{Proposition 4.4.}

(a) For every pointed 2-cocycle $z \in \mathrm{Z}_{\mathrm{pt}}^{2}(E, M)$, we have

$$
(m) z_{\mathrm{Mp}}^{\mathrm{st}}=\left(m[m]^{-1}\right) z_{\mathrm{Mp}}
$$

for $m \in \operatorname{Mp} E$, and

$$
\begin{gathered}
(h, g) z_{\mathrm{Gp}}^{\mathrm{st}}=\left(\left[h[\bar{h}]^{-1}\right]^{-1 h}\left(\left[g[\bar{g}]^{-1}\right]^{-1}\right)\left[h g[\overline{h g}]^{-1}\right]\right) z_{\mathrm{Mp}}-([\bar{h}, \bar{g}],[\bar{h} \bar{g}]) z_{\mathrm{Gp}} \\
+([\bar{h}],[\bar{g}]) z_{\mathrm{Gp}}
\end{gathered}
$$

for $g, h \in \mathrm{Gp} E$.

(b) For every pointed 2-coboundary $b \in \mathrm{B}_{\mathrm{pt}}^{2}(E, M)$, we have

$$
(m) b_{\mathrm{Mp}}^{\mathrm{st}}=0
$$

for $m \in \mathrm{Mp} E$, and, given $c \in \mathrm{Ch}_{\mathrm{pt}}^{1}(E, M)$ with $b=c \partial$, we have

$$
(h, g) b_{\mathrm{Gp}}^{\mathrm{st}}=(\bar{h}, \bar{g})\left(c_{0} \partial\right)
$$

for $g, h \in \mathrm{Gp} E$, where $c_{0} \in \mathrm{Ch}^{1}\left(\Pi_{0}, M\right)$ is given by $(p) c_{0}:=([p])$.

\section{Proof.}

(a) We suppose given a pointed 2-cocycle $z \in \mathrm{Z}_{\mathrm{pt}}^{2}(E, M)$. By proposition 3.11(b), we have

$$
\begin{aligned}
(m) z_{\mathrm{Mp}}^{\mathrm{st}} & =(m) z_{\mathrm{Mp}}-(m)\left(\mathrm{s}_{z} \partial\right)_{\mathrm{Mp}}=(m) z_{\mathrm{Mp}}-(m) \mathrm{s}_{z} \\
& =(m) z_{\mathrm{Mp}}-([m]) z_{\mathrm{Mp}}=(m) z_{\mathrm{Mp}}+\left([m]^{-1}\right) z_{\mathrm{Mp}}-\left(m, m^{-1}\right) z_{\mathrm{Gp}} \\
& =\left(m[m]^{-1}\right) z_{\mathrm{Mp}}
\end{aligned}
$$

for $m \in \operatorname{Mp} E$, and

$$
\begin{aligned}
(h, g) z_{\mathrm{Gp}}^{\mathrm{st}}= & (h, g) z_{\mathrm{Gp}}-(h, g)\left(\mathrm{s}_{z} \partial\right)_{\mathrm{Gp}} \\
= & (h, g) z_{\mathrm{Gp}}-(h) \mathrm{s}_{z}+(h g) \mathrm{s}_{z}-\bar{h} \cdot(g) \mathrm{s}_{z} \\
= & (h, g) z_{\mathrm{Gp}}-\left(\left[h[\bar{h}]^{-1}\right]\right) z_{\mathrm{Mp}}+\left(h[\bar{h}]^{-1},[\bar{h}]\right) z_{\mathrm{Gp}}+\left(\left[h g[\overline{h g}]^{-1}\right]\right) z_{\mathrm{Mp}} \\
& \quad-\left(h g[\overline{h g}]^{-1},[\overline{h g}]\right) z_{\mathrm{Gp}}-\bar{h} \cdot\left(\left[g[\bar{g}]^{-1}\right]\right) z_{\mathrm{Mp}}+\bar{h} \cdot\left(g[\bar{g}]^{-1},[\bar{g}]\right) z_{\mathrm{Gp}} \\
= & (h, g) z_{\mathrm{Gp}}+\left(\left[h[\bar{h}]^{-1}\right]^{-1}\right) z_{\mathrm{Mp}}-\left(h[\bar{h}]^{-1},[\bar{h}] h^{-1}\right) z_{\mathrm{Gp}}+\left(h[\bar{h}]^{-1},[\bar{h}]\right) z_{\mathrm{Gp}} \\
& +\left(\left[h g[\overline{h g}]^{-1}\right]\right) z_{\mathrm{Mp}}-\left(h g[\overline{h g}]^{-1},[\overline{h g}]\right) z_{\mathrm{Gp}}+\bar{h} \cdot\left(\left[g[\bar{g}]^{-1}\right]^{-1}\right) z_{\mathrm{Mp}} \\
& -\bar{h} \cdot\left(g[\bar{g}]^{-1},[\bar{g}] g^{-1}\right) z_{\mathrm{Gp}}+\bar{h} \cdot\left(g[\bar{g}]^{-1},[\bar{g}]\right) z_{\mathrm{Gp}} \\
= & (h, g) z_{\mathrm{Gp}}+\left(\left[h[\bar{h}]^{-1}\right]^{-1}\right) z_{\mathrm{Mp}}-\left(h[\bar{h}]^{-1},[\bar{h}] h^{-1}\right) z_{\mathrm{Gp}}+\left(h[\bar{h}]^{-1},[\bar{h}]\right) z_{\mathrm{Gp}} \\
& +\left(\left[h g[\overline{h g}]^{-1}\right]\right) z_{\mathrm{Mp}}-\left(h g[\overline{h g}]^{-1},[\overline{h g}]\right) z_{\mathrm{Gp}}+\left({ }^{h}\left(\left[g[\bar{g}]^{-1}\right]^{-1}\right)\right) z_{\mathrm{Mp}} \\
& -\left({ }^{h}\left([\bar{g}] g^{-1}\right), h\right) z_{\mathrm{Gp}}+\left(h,[\bar{g}] g^{-1}\right) z_{\mathrm{Gp}}-\bar{h} \cdot\left(g[\bar{g}]^{-1},[\bar{g}] g^{-1}\right) z_{\mathrm{Gp}} \\
& +\bar{h} \cdot\left(g[\bar{g}]^{-1},[\bar{g}]\right) z_{\mathrm{Gp}}
\end{aligned}
$$




$$
\begin{aligned}
& =\left(\left[h[\bar{h}]^{-1}\right]^{-1}\right) z_{\mathrm{Mp}}+\left({ }^{h}\left(\left[g[\bar{g}]^{-1}\right]^{-1}\right)\right) z_{\mathrm{Mp}}+\left(\left[h g[\overline{h g}]^{-1}\right]\right) z_{\mathrm{Mp}}+(h, g) z_{\mathrm{Gp}} \\
& -\left(h[\bar{h}]^{-1},[\bar{h}] h^{-1}\right) z_{\mathrm{Gp}}+\left(h[\bar{h}]^{-1},[\bar{h}]\right) z_{\mathrm{Gp}}-\left(h g[\overline{h g}]^{-1},[\overline{h g}]\right) z_{\mathrm{Gp}} \\
& -\left({ }^{h}\left([\bar{g}] g^{-1}\right), h\right) z_{\mathrm{Gp}}+\left(h,[\bar{g}] g^{-1}\right) z_{\mathrm{Gp}}-\bar{h} \cdot\left(g[\bar{g}]^{-1},[\bar{g}] g^{-1}\right) z_{\mathrm{Gp}} \\
& +\bar{h} \cdot\left(g[\bar{g}]^{-1},[\bar{g}]\right) z_{\mathrm{Gp}} \\
& =\left(\left[h[\bar{h}]^{-1}\right]^{-1}\right) z_{\mathrm{Mp}}+\left({ }^{h}\left(\left[g[\bar{g}]^{-1}\right]^{-1}\right)\left[h g[\overline{h g}]^{-1}\right]\right) z_{\mathrm{Mp}} \\
& +\left(h[\bar{g}] g^{-1} h^{-1}, h g[\overline{h g}]^{-1}\right) z_{\mathrm{Gp}}+(h, g) z_{\mathrm{Gp}}-\left(h[\bar{h}]^{-1},[\bar{h}] h^{-1}\right) z_{\mathrm{Gp}} \\
& +\left(h[\bar{h}]^{-1},[\bar{h}]\right) z_{\mathrm{Gp}}-\left(h g[\overline{h g}]^{-1},[\overline{h g}]\right) z_{\mathrm{Gp}}-\left({ }^{h}\left([\bar{g}] g^{-1}\right), h\right) z_{\mathrm{Gp}} \\
& +\left(h,[\bar{g}] g^{-1}\right) z_{\mathrm{Gp}}-\bar{h} \cdot\left(g[\bar{g}]^{-1},[\bar{g}] g^{-1}\right) z_{\mathrm{Gp}}+\bar{h} \cdot\left(g[\bar{g}]^{-1},[\bar{g}]\right) z_{\mathrm{Gp}} \\
& =\left(\left[h[\bar{h}]^{-1}\right]^{-1} h\left(\left[g[\bar{g}]^{-1}\right]^{-1}\right)\left[h g[\overline{h g}]^{-1}\right]\right) z_{\mathrm{Mp}}+\left([\bar{h}] h^{-1}, h[\bar{g}][\overline{h g}]^{-1}\right) z_{\mathrm{Gp}} \\
& +\left(h[\bar{g}] g^{-1} h^{-1}, h g[\overline{h g}]^{-1}\right) z_{\mathrm{Gp}}+(h, g) z_{\mathrm{Gp}}-\left(h[\bar{h}]^{-1},[\bar{h}] h^{-1}\right) z_{\mathrm{Gp}} \\
& +\left(h[\bar{h}]^{-1},[\bar{h}]\right) z_{\mathrm{Gp}}-\left(h g[\overline{h g}]^{-1},[\overline{h g}]\right) z_{\mathrm{Gp}}-\left(h[\bar{g}] g^{-1} h^{-1}, h\right) z_{\mathrm{Gp}} \\
& +\left(h,[\bar{g}] g^{-1}\right) z_{\mathrm{Gp}}-\bar{h} \cdot\left(g[\bar{g}]^{-1},[\bar{g}] g^{-1}\right) z_{\mathrm{Gp}}+\bar{h} \cdot\left(g[\bar{g}]^{-1},[\bar{g}]\right) z_{\mathrm{Gp}} \\
& =\left(\left[h[\bar{h}]^{-1}\right]^{-1} h\left(\left[g[\bar{g}]^{-1}\right]^{-1}\right)\left[h g[\overline{h g}]^{-1}\right]\right) z_{\mathrm{Mp}}+\left([\bar{h}][\bar{g}],[\overline{h g}]^{-1}\right) z_{\mathrm{Gp}} \\
& -\left(h[\bar{g}],[\overline{h g}]^{-1}\right) z_{\mathrm{Gp}}+\left([\bar{h}] h^{-1}, h[\bar{g}]\right) z_{\mathrm{Gp}}+\left(h[\bar{g}],[\overline{h g}]^{-1}\right) z_{\mathrm{Gp}} \\
& -\left(h g,[\overline{h g}]^{-1}\right) z_{\mathrm{Gp}}+\left(h[\bar{g}] g^{-1} h^{-1}, h g\right) z_{\mathrm{Gp}}+(h, g) z_{\mathrm{Gp}} \\
& +\left(h g,[\overline{h g}]^{-1}\right) z_{\mathrm{Gp}}-\left(h, h^{-1}\right) z_{\mathrm{Gp}}+\left([\bar{h}], h^{-1}\right) z_{\mathrm{Gp}} \\
& -\overline{h g} \cdot\left([\overline{h g}]^{-1},[\overline{h g}]\right) z_{\mathrm{Gp}}-\left(h[\bar{g}], g^{-1}\right) z_{\mathrm{Gp}}+\left(h[\bar{g}], g^{-1} h^{-1}\right) z_{\mathrm{Gp}} \\
& -\overline{h g} \cdot\left(g^{-1} h^{-1}, h\right) z_{\mathrm{Gp}}+\left(h[\bar{g}], g^{-1}\right) z_{\mathrm{Gp}}-\bar{h} \cdot\left([\bar{g}], g^{-1}\right) z_{\mathrm{Gp}} \\
& +(h,[\bar{g}]) z_{\mathrm{Gp}}-\bar{h} \cdot\left(g, g^{-1}\right) z_{\mathrm{Gp}}+\bar{h} \cdot\left([\bar{g}], g^{-1}\right) z_{\mathrm{Gp}} \\
& =\left(\left[h[\bar{h}]^{-1}\right]^{-1 h}\left(\left[g[\bar{g}]^{-1}\right]^{-1}\right)\left[h g[\overline{h g}]^{-1}\right]\right) z_{\mathrm{Mp}}+\left([\bar{h}][\bar{g}],[\overline{h g}]^{-1}\right) z_{\mathrm{Gp}} \\
& +\left([\bar{h}] h^{-1}, h[\bar{g}]\right) z_{\mathrm{Gp}}+\left(h[\bar{g}] g^{-1} h^{-1}, h g\right) z_{\mathrm{Gp}}+(h, g) z_{\mathrm{Gp}} \\
& -\left(h, h^{-1}\right) z_{\mathrm{Gp}}+\left([\bar{h}], h^{-1}\right) z_{\mathrm{Gp}}-\overline{h g} \cdot\left([\overline{h g}]^{-1},[\overline{h g}]\right) z_{\mathrm{Gp}} \\
& +\left(h[\bar{g}], g^{-1} h^{-1}\right) z_{\mathrm{Gp}}-\overline{h g} \cdot\left(g^{-1} h^{-1}, h\right) z_{\mathrm{Gp}}+(h,[\bar{g}]) z_{\mathrm{Gp}} \\
& -\bar{h} \cdot\left(g, g^{-1}\right) z_{\mathrm{Gp}} \\
& =\left(\left[h[\bar{h}]^{-1}\right]^{-1 h}\left(\left[g[\bar{g}]^{-1}\right]^{-1}\right)\left[h g[\overline{h g}]^{-1}\right]\right) z_{\mathrm{Mp}}+\left([\bar{h}][\bar{g}],[\overline{h g}]^{-1}\right) z_{\mathrm{Gp}} \\
& +([\bar{h}],[\bar{g}]) z_{\mathrm{Gp}}-(h,[\bar{g}]) z_{\mathrm{Gp}}+\left([\bar{h}] h^{-1}, h\right) z_{\mathrm{Gp}}-\left(h[\bar{g}], g^{-1} h^{-1}\right) z_{\mathrm{Gp}} \\
& +\overline{h g} \cdot\left(g^{-1} h^{-1}, h g\right) z_{\mathrm{Gp}}+(h, g) z_{\mathrm{Gp}}-\left(h, h^{-1}\right) z_{\mathrm{Gp}}+\left([\bar{h}], h^{-1}\right) z_{\mathrm{Gp}} \\
& -\overline{h g} \cdot\left([\overline{h g}]^{-1},[\overline{h g}]\right) z_{\mathrm{Gp}}+\left(h[\bar{g}], g^{-1} h^{-1}\right) z_{\mathrm{Gp}}-\overline{h g} \cdot\left(g^{-1} h^{-1}, h\right) z_{\mathrm{Gp}} \\
& +(h,[\bar{g}]) z_{\mathrm{Gp}}-\bar{h} \cdot\left(g, g^{-1}\right) z_{\mathrm{Gp}} \\
& =\left(\left[h[\bar{h}]^{-1}\right]^{-1} h\left(\left[g[\bar{g}]^{-1}\right]^{-1}\right)\left[h g[\overline{h g}]^{-1}\right]\right) z_{\mathrm{Mp}}+\left([\bar{h}][\bar{g}],[\overline{h g}]^{-1}\right) z_{\mathrm{Gp}} \\
& +([\bar{h}],[\bar{g}]) z_{\mathrm{Gp}}+\left([\bar{h}] h^{-1}, h\right) z_{\mathrm{Gp}}+\overline{h g} \cdot\left(g^{-1} h^{-1}, h g\right) z_{\mathrm{Gp}}+(h, g) z_{\mathrm{Gp}} \\
& -\left(h, h^{-1}\right) z_{\mathrm{Gp}}+\left([\bar{h}], h^{-1}\right) z_{\mathrm{Gp}}-\overline{h g} \cdot\left([\overline{h g}]^{-1},[\overline{h g}]\right) z_{\mathrm{Gp}} \\
& -\overline{h g} \cdot\left(g^{-1} h^{-1}, h\right) z_{\mathrm{Gp}}-\bar{h} \cdot\left(g, g^{-1}\right) z_{\mathrm{Gp}}
\end{aligned}
$$




$$
\begin{aligned}
=\left(\left[h[\bar{h}]^{-1}\right]^{-1 h}\left(\left[g[\bar{g}]^{-1}\right]^{-1}\right)\left[h g[\overline{h g}]^{-1}\right]\right) z_{\mathrm{Mp}}+\left([\bar{h}][\bar{g}],[\overline{h g}]^{-1}\right) z_{\mathrm{Gp}} \\
\quad+([\bar{h}],[\bar{g}]) z_{\mathrm{Gp}}-\left([\bar{h}], h^{-1}\right) z_{\mathrm{Gp}}+\bar{h} \cdot\left(h^{-1}, h\right) z_{\mathrm{Gp}}+\overline{h g} \cdot\left(g^{-1}, g\right) z_{\mathrm{Gp}} \\
\quad-(h, g) z_{\mathrm{Gp}}+\overline{h g} \cdot\left(g^{-1} h^{-1}, h\right) z_{\mathrm{Gp}}+(h, g) z_{\mathrm{Gp}}-\left(h, h^{-1}\right) z_{\mathrm{Gp}} \\
\quad+\left([\bar{h}], h^{-1}\right) z_{\mathrm{Gp}}-\overline{h g} \cdot\left([\overline{h g}]^{-1},[\overline{h g}]\right) z_{\mathrm{Gp}}-\overline{h g} \cdot\left(g^{-1} h^{-1}, h\right) z_{\mathrm{Gp}} \\
\quad-\bar{h} \cdot\left(g, g^{-1}\right) z_{\mathrm{Gp}} \\
=\left(\left[h[\bar{h}]^{-1}\right]^{-1 h}\left(\left[g[\bar{g}]^{-1}\right]^{-1}\right)\left[h g[\overline{h g}]^{-1}\right]\right) z_{\mathrm{Mp}}+\left([\bar{h}][\bar{g}],[\overline{h g}]^{-1}\right) z_{\mathrm{Gp}} \\
\quad+([\bar{h}],[\bar{g}]) z_{\mathrm{Gp}}+\bar{h} \cdot\left(h^{-1}, h\right) z_{\mathrm{Gp}}+\overline{h g} \cdot\left(g^{-1}, g\right) z_{\mathrm{Gp}} \\
\quad-\left(h, h^{-1}\right) z_{\mathrm{Gp}}-\overline{h g} \cdot\left([\overline{h g}]^{-1},[\overline{h g}]\right) z_{\mathrm{Gp}}-\bar{h} \cdot\left(g, g^{-1}\right) z_{\mathrm{Gp}} \\
=\left(\left[h[\bar{h}]^{-1}\right]^{-1} h\left(\left[g[\bar{g}]^{-1}\right]^{-1}\right)\left[h g[\overline{h g}]^{-1}\right]\right) z_{\mathrm{Mp}}+\left([\bar{h}][\bar{g}],[\overline{h g}]^{-1}\right) z_{\mathrm{Gp}} \\
\quad+([\bar{h}],[\bar{g}]) z_{\mathrm{Gp}}+\left(h, h^{-1}\right) z_{\mathrm{Gp}}+\bar{h} \cdot\left(g, g^{-1}\right) z_{\mathrm{Gp}}-\left(h, h^{-1}\right) z_{\mathrm{Gp}} \\
\quad-\overline{h g} \cdot\left([\overline{h g}]^{-1},[\overline{h g}]\right) z_{\mathrm{Gp}}-\bar{h} \cdot\left(g, g^{-1}\right) z_{\mathrm{Gp}} \\
=\left(\left[h[\bar{h}]^{-1}\right]^{-1} h\left(\left[g[\bar{g}]^{-1}\right]^{-1}\right)\left[h g[\overline{h g}]^{-1}\right]\right) z_{\mathrm{Mp}}+\left([\bar{h}][\bar{g}],[\overline{h g}]^{-1}\right) z_{\mathrm{Gp}} \\
\quad-\overline{h g} \cdot\left([\overline{h g}]^{-1},[\overline{h g}]\right) z_{\mathrm{Gp}}+([\bar{h}],[\bar{g}]) z_{\mathrm{Gp}} \\
=\left(\left[h[\bar{h}]^{-1}\right]^{-1 h}\left(\left[g[\bar{g}]^{-1}\right]^{-1}\right)\left[h g[\overline{h g}]^{-1}\right]\right) z_{\mathrm{Mp}}-\left([\bar{h}][\bar{g}][\overline{h g}]^{-1},[\overline{h g}]\right) z_{\mathrm{Gp}} \\
\quad+([\bar{h}],[\bar{g}]) z_{\mathrm{Gp}} \\
\quad\left(\left[h[\bar{h}]^{-1}\right]^{-1 h}\left(\left[g[\bar{g}]^{-1}\right]^{-1}\right)\left[h g[\overline{h g}]^{-1}\right]\right) z_{\mathrm{Mp}}-([\bar{h}, \bar{g}],[\overline{h g}]) z_{\mathrm{Gp}} \\
\quad+([\bar{h}],[\bar{g}]) z_{\mathrm{Gp}}
\end{aligned}
$$

for $g, h \in \mathrm{Gp} E$.

(b) By (a), we have

$$
(m) b_{\mathrm{Mp}}^{\mathrm{st}}=\left(m[m]^{-1}\right) b_{\mathrm{Mp}}=\left(m[m]^{-1}\right)(c \partial)_{\mathrm{Mp}}=\left(m m^{-1}\right) c=0
$$

for $m \in \mathrm{Mp} E$ and

$$
\begin{aligned}
(h, g) b_{\mathrm{Gp}}^{\mathrm{st}}= & \left(\left[h[\bar{h}]^{-1}\right]^{-1 h}\left(\left[g[\bar{g}]^{-1}\right]^{-1}\right)\left[h g[\overline{h g}]^{-1}\right]\right) b_{\mathrm{Mp}}-([\bar{h}, \bar{g}],[\bar{h} \bar{g}]) b_{\mathrm{Gp}} \\
& \quad+([\bar{h}],[\bar{g}]) b_{\mathrm{Gp}} \\
= & \left(\left[h[\bar{h}]^{-1}\right]^{-1} h\left(\left[g[\bar{g}]^{-1}\right]^{-1}\right)\left[h g[\overline{h g}]^{-1}\right]\right)(c \partial)_{\mathrm{Mp}}-([\bar{h}, \bar{g}],[\bar{h} \bar{g}])(c \partial)_{\mathrm{Gp}} \\
& \quad+([\bar{h}],[\bar{g}])(c \partial)_{\mathrm{Gp}} \\
= & \left(\left(h[\bar{h}]^{-1}\right)^{-1} h\left(\left(g[\bar{g}]^{-1}\right)^{-1}\right)\left(h g[\overline{h g}]^{-1}\right)\right) c-([\bar{h}, \bar{g}]) c+([\bar{h}, \bar{g}][\bar{h} \bar{g}]) c \\
& \quad-([\bar{h} \bar{g}]) c+([\bar{h}]) c-([\bar{h}][\bar{g}]) c+\bar{h} \cdot([\bar{g}]) c \\
= & -([\bar{h} \bar{g}]) c+([\bar{h}]) c+\bar{h} \cdot([\bar{g}]) c=(\bar{h}) c_{0}-(\bar{h} \bar{g}) c_{0}+\bar{h} \cdot(\bar{g}) c_{0}=(\bar{h}, \bar{g})\left(c_{0} \partial\right)
\end{aligned}
$$

for $g, h \in \mathrm{Gp} E$.

\section{Corollary 4.5.}

(a) Given a pointed 2-cocycle $z \in \mathrm{Z}_{\mathrm{pt}}^{2}(E, M)$, we have

$$
([m]) z_{\mathrm{Mp}}^{\mathrm{st}}=\left(g[\bar{g}]^{-1},[\bar{g}]\right) z_{\mathrm{Gp}}^{\mathrm{st}}=0
$$

for $m \in \operatorname{Mp} E, g \in \mathrm{Gp} E$. 
(b) We have

$$
\begin{aligned}
\mathrm{Z}_{\mathrm{st}}^{2}(E, M)=\left\{z \in \mathrm{Z}_{\mathrm{pt}}^{2}(E, M) \mid([m]) z_{\mathrm{Mp}}=\left(g[\bar{g}]^{-1},[\bar{g}]\right) z_{\mathrm{Gp}}=0\right. \\
\\
\text { for all } m \in \mathrm{Mp} E, g \in \mathrm{Gp} E\} .
\end{aligned}
$$

In particular, the standardisation $z^{\text {st }}$ of every $z \in \mathrm{Z}^{2}(E, M)$ is standard.

(c) The embedding $\mathrm{Z}_{\mathrm{st}}^{2}(E, M) \rightarrow \mathrm{Z}_{\mathrm{pt}}^{2}(E, M)$ and the standardisation homomorphism $\mathrm{Z}_{\mathrm{pt}}^{2}(E, M) \rightarrow \mathrm{Z}_{\mathrm{st}}^{2}(E, M), z \mapsto z^{\mathrm{st}}$ induce mutually inverse isomorphisms between $\mathrm{H}_{\mathrm{st}}^{2}(E, M)$ and $\mathrm{H}_{\mathrm{pt}}^{2}(E, M)$. In particular,

$$
\mathrm{H}^{2}(E, M) \cong \mathrm{H}_{\mathrm{st}}^{2}(E, M)
$$

Proof.

(a) We suppose given a pointed 2-cocycle $z \in \mathrm{Z}_{\mathrm{pt}}^{2}(E, M)$. Proposition 4.4(a) implies

$$
([m]) z_{\mathrm{Mp}}^{\mathrm{st}}=\left([m][m]^{-1}\right) z_{\mathrm{Mp}}=0
$$

for $m \in \operatorname{Mp} E$ and

$$
\begin{aligned}
\left(g[\bar{g}]^{-1},[\bar{g}]\right) z_{\mathrm{Gp}}^{\mathrm{st}} & =\left(\left[g[\bar{g}]^{-1}\right]^{-1}\left[g[\bar{g}]^{-1}\right]\right) z_{\mathrm{Mp}}-([1, \bar{g}],[\bar{g}]) z_{\mathrm{Gp}}+(1,[\bar{g}]) z_{\mathrm{Gp}} \\
& =0
\end{aligned}
$$

for $g \in \operatorname{Gp} E$.

(b) Given a standard 2-cocycle $z \in \mathrm{Z}_{\mathrm{st}}^{2}(E, M)$, we have $([m]) z_{\mathrm{Mp}}=([m]) z_{\mathrm{Mp}}^{\mathrm{st}}=0$ for all $m \in \operatorname{Mp} E$ and $\left(g[\bar{g}]^{-1},[\bar{g}]\right) z_{\mathrm{Gp}}=\left(g[\bar{g}]^{-1},[\bar{g}]\right) z_{\mathrm{Gp}}^{\mathrm{st}}=0$ for all $g \in \mathrm{Gp} E$ by (a). Conversely, given a pointed 2-cocycle $z \in \mathrm{Z}_{\mathrm{pt}}^{2}(E, M)$ with $([m]) z_{\mathrm{Mp}}=$ $\left(g[\bar{g}]^{-1},[\bar{g}]\right) z_{\mathrm{Gp}}=0$ for all $m \in \mathrm{Mp} E, g \in \mathrm{Gp} E$, it follows that

$$
(g) \mathrm{s}_{z}=\left(\left[g[\bar{g}]^{-1}\right]\right) z_{\mathrm{Mp}}-\left(g[\bar{g}]^{-1},[\bar{g}]\right) z_{\mathrm{Gp}}=0
$$

for all $g \in \operatorname{Gp} E$, that is, $\mathrm{s}_{z}=0$. Hence $z^{\mathrm{st}}=z-\mathrm{s}_{z} \partial=z$, that is, $z$ is standard. Altogether, we have

$$
\begin{aligned}
\mathrm{Z}_{\mathrm{st}}^{2}(E, M)=\{ & \left\{\in \mathrm{Z}_{\mathrm{pt}}^{2}(E, M) \mid([m]) z_{\mathrm{Mp}}=\left(g[\bar{g}]^{-1},[\bar{g}]\right) z_{\mathrm{Gp}}=0\right. \\
& \text { for all } m \in \mathrm{Mp} E, g \in \mathrm{Gp} E\}
\end{aligned}
$$

and a further application of (a) shows that $z^{\text {st }} \in \mathrm{Z}_{\mathrm{st}}^{2}(E, M)$ for all $z \in \mathrm{Z}^{2}(E, M)$.

(c) By definition of the standardisation, we have $z=z^{\text {st }}+\mathrm{s}_{z} \partial$ for every pointed 2-cocycle $z \in \mathrm{Z}_{\mathrm{pt}}^{2}(E, M)$ and since the standardisation $z^{\text {st }}$ is standard by (b), it follows that

$$
\begin{aligned}
\mathrm{H}_{\mathrm{pt}}^{2}(E, M) & =\mathrm{Z}_{\mathrm{pt}}^{2}(E, M) / \mathrm{B}_{\mathrm{pt}}^{2}(E, M) \\
& =\left(\mathrm{Z}_{\mathrm{st}}^{2}(E, M)+\mathrm{B}_{\mathrm{pt}}^{2}(E, M)\right) / \mathrm{B}_{\mathrm{pt}}^{2}(E, M) .
\end{aligned}
$$

Moreover,

$$
\begin{aligned}
\mathrm{H}_{\mathrm{st}}^{2}(E, M) & =\mathrm{Z}_{\mathrm{st}}^{2}(E, M) / \mathrm{B}_{\mathrm{st}}^{2}(E, M) \\
& =\mathrm{Z}_{\mathrm{st}}^{2}(E, M) /\left(\mathrm{Z}_{\mathrm{st}}^{2}(E, M) \cap \mathrm{B}_{\mathrm{pt}}^{2}(E, M)\right),
\end{aligned}
$$

and thus Noether's first law of isomorphism provides the asserted isomorphisms

$$
\mathrm{H}_{\mathrm{st}}^{2}(E, M) \rightarrow \mathrm{H}_{\mathrm{pt}}^{2}(E, M), z+\mathrm{B}_{\mathrm{st}}^{2}(E, M) \mapsto z+\mathrm{B}_{\mathrm{pt}}^{2}(E, M)
$$


and

$$
\mathrm{H}_{\mathrm{pt}}^{2}(E, M) \rightarrow \mathrm{H}_{\mathrm{st}}^{2}(E, M), z+\mathrm{B}_{\mathrm{pt}}^{2}(E, M) \mapsto z^{\mathrm{st}}+\mathrm{B}_{\mathrm{st}}^{2}(E, M) .
$$

In particular, we have

$$
\mathrm{H}^{2}(E, M) \cong \mathrm{H}_{\mathrm{pt}}^{2}(E, M) \cong \mathrm{H}_{\mathrm{st}}^{2}(E, M),
$$

cf. section 2.12 .

Similarly to proposition 3.11 , we will give in proposition 4.7 a characterisation of standard 2-cocycles and 2-coboundaries. For convenience, we introduce the following abbreviation first.

Notation 4.6. For $g, h \in \mathrm{Gp} E$, we abbreviate

$$
(h, g) \kappa:=\left[h[\bar{h}]^{-1}\right]^{-1} h\left(\left[g[\bar{g}]^{-1}\right]^{-1}\right)\left[h g[\overline{h g}]^{-1}\right][\bar{h}, \bar{g}]^{-1} \in \operatorname{Ker} \mu .
$$

\section{Proposition 4.7.}

(a) A pointed 2-cochain $z \in \mathrm{Ch}_{\mathrm{pt}}^{2}(E, M)$ is a standard 2-cocycle if and only if the following conditions hold:

(i) We have $(m, h, g) z=(m) z_{\mathrm{Mp}}-(m, h) z_{\mathrm{Gp}}+(h, g) z_{\mathrm{Gp}}$ for $m \in \mathrm{Mp} V, g, h \in$ $\mathrm{Gp} V$.

(ii) We have $(m) z_{\mathrm{Mp}}=\left(m[m]^{-1}\right) z_{\mathrm{Mp}}$ for $m \in \mathrm{Mp} E$.

(iii) We have $(h, g) z_{\mathrm{Gp}}=((h, g) \kappa) z_{\mathrm{Mp}}+([\bar{h}],[\bar{g}]) z_{\mathrm{Gp}}$ for $g, h \in \mathrm{Gp} E$.

(iv) We have $\mathrm{\iota} z_{\mathrm{Mp}} \in \operatorname{Hom}_{\Pi_{0}}\left(\Pi_{1}, M\right)$.

(v) We have $([r, q, p] \iota) z_{\mathrm{Mp}}=(r, q, p)\left(\left(\left(s^{0} \times s^{0}\right) z_{\mathrm{Gp}}\right) \partial\right)$ for $p, q, r \in \Pi_{0}$.

(b) A pointed 2-cochain $b \in \mathrm{Ch}_{\mathrm{pt}}^{2}(E, M)$ is a standard 2-coboundary if and only if the following conditions hold:

(i) We have $b_{\mathrm{Mp}}=0$.

(ii) There exists a pointed 1-cochain $c_{0} \in \mathrm{Ch}_{\mathrm{pt}}^{1}\left(\Pi_{0}, M\right)$ such that $(h, g) b_{\mathrm{Gp}}=$ $(\bar{h}, \bar{g})\left(c_{0} \partial\right)$ for $g, h \in \mathrm{Gp} E$.

Proof.

(a) First, we suppose given a standard 2-cocycle $z \in \mathrm{Z}_{\mathrm{st}}^{2}(E, M)$. We verify the asserted formulas:

(i) Since $z$ is a 2-cocycle, this property holds by proposition 3.11(b)(i).

(ii) By corollary 4.5(b), we have

$$
\begin{aligned}
(m) z_{\mathrm{Mp}} & =\left(m[m]^{-1}[m]\right) z_{\mathrm{Mp}}=\left(m[m]^{-1}\right) z_{\mathrm{Mp}}+([m]) z_{\mathrm{Mp}}-(1, m) z_{\mathrm{Gp}} \\
& =\left(m[m]^{-1}\right) z_{\mathrm{Mp}}
\end{aligned}
$$

for $m \in \operatorname{Mp} E$.

(iii) By proposition 4.4(a), proposition 3.11(b)(iii), corollary 4.5(b) and (ii), we have

$$
\begin{aligned}
(h, g) z_{\mathrm{Gp}}= & (h, g) z_{\mathrm{Gp}}^{\mathrm{st}} \\
= & \left(\left[h[\bar{h}]^{-1}\right]^{-1 h}\left(\left[g[\bar{g}]^{-1}\right]^{-1}\right)\left[h g[\overline{h g}]^{-1}\right]\right) z_{\mathrm{Mp}}-([\bar{h}, \bar{g}],[\bar{h} \bar{g}]) z_{\mathrm{Gp}} \\
& +([\bar{h}],[\bar{g}]) z_{\mathrm{Gp}}
\end{aligned}
$$




$$
\begin{aligned}
= & \left(\left(\left[h[\bar{h}]^{-1}\right]^{-1} h\left(\left[g[\bar{g}]^{-1}\right]^{-1}\right)\left[h g[\overline{h g}]^{-1}\right][\bar{h}, \bar{g}]^{-1}\right)[\bar{h}, \bar{g}]\right) z_{\mathrm{Mp}} \\
& -\left([\bar{h}][\bar{g}][\bar{h} \bar{g}]^{-1},[\bar{h} \bar{g}]\right) z_{\mathrm{Gp}}+([\bar{h}],[\bar{g}]) z_{\mathrm{Gp}} \\
= & \left(\left[h[\bar{h}]^{-1}\right]^{-1} h\left(\left[g[\bar{g}]^{-1}\right]^{-1}\right)\left[h g[\overline{h g}]^{-1}\right][\bar{h}, \bar{g}]^{-1}\right) z_{\mathrm{Mp}}+([\bar{h}],[\bar{g}]) z_{\mathrm{Gp}} \\
= & ((h, g) \kappa) z_{\mathrm{Mp}}+([\bar{h}],[\bar{g}]) z_{\mathrm{Gp}}
\end{aligned}
$$

for $g, h \in \mathrm{Gp} E$.

(iv) We have $\left\llcorner z_{\mathrm{Mp}} \in \operatorname{Hom}_{\Pi_{0}}\left(\Pi_{1}, M\right)\right.$ by proposition 3.11(b)(iii) and (iv).

(v) Using proposition 3.11(b) and corollary 4.5(b), we compute

$$
\begin{aligned}
& ([r, q, p] \iota) z_{\mathrm{Mp}}=\left([r, q][r q, p][r, q p]^{-1}\left({ }^{[r]}[q, p]\right)^{-1}\right) z_{\mathrm{Mp}} \\
& =([r, q][r q, p]) z_{\mathrm{Mp}}-\left({ }^{[r]}[q, p][r, q p]\right) z_{\mathrm{Mp}} \\
& =([r, q]) z_{\mathrm{Mp}}+([r q, p]) z_{\mathrm{Mp}}-([r, q],[r q, p]) z_{\mathrm{Gp}}-\left({ }^{[r]}[q, p]\right) z_{\mathrm{Mp}} \\
& -([r, q p]) z_{\mathrm{Mp}}+\left({ }^{[r]}[q, p],[r, q p]\right) z_{\mathrm{Gp}} \\
& =-([r, q],[r q, p]) z_{\mathrm{Gp}}-r \cdot([q, p]) z_{\mathrm{Mp}}-\left({ }^{[r]}[q, p],[r]\right) z_{\mathrm{Gp}} \\
& +([r],[q, p]) z_{\mathrm{Gp}}+\left({ }^{[r]}[q, p],[r, q p]\right) z_{\mathrm{Gp}} \\
& =-\left([r, q],[r q][p][r q p]^{-1}\right) z_{\mathrm{Gp}}-\left([r][q, p][r]^{-1},[r]\right) z_{\mathrm{Gp}}+([r],[q, p]) z_{\mathrm{Gp}} \\
& +\left([r][q, p][r]^{-1},[r][q p][r q p]^{-1}\right) z_{\mathrm{Gp}} \\
& =-\left([r, q][r q],[p][r q p]^{-1}\right) z_{\mathrm{Gp}}+\left([r q],[p][r q p]^{-1}\right) z_{\mathrm{Gp}}-([r, q],[r q]) z_{\mathrm{Gp}} \\
& +\left([r],[q, p][r]^{-1}\right) z_{\mathrm{Gp}}-r \cdot\left([q, p][r]^{-1},[r]\right) z_{\mathrm{Gp}} \\
& +\left([r],[q, p][q p][r q p]^{-1}\right) z_{\mathrm{Gp}}-\left([r],[q, p][r]^{-1}\right) z_{\mathrm{Gp}} \\
& +r \cdot\left([q, p][r]^{-1},[r][q p][r q p]^{-1}\right) z_{\mathrm{Gp}} \\
& =-\left([r][q],[p][r q p]^{-1}\right) z_{\mathrm{Gp}}+\left([r q],[p][r q p]^{-1}\right) z_{\mathrm{Gp}} \\
& -\left([r][q][r q]^{-1},[r q]\right) z_{\mathrm{Gp}}-r \cdot\left([q, p][r]^{-1},[r]\right) z_{\mathrm{Gp}} \\
& +\left([r],[q][p][r q p]^{-1}\right) z_{\mathrm{Gp}}+r \cdot\left([q, p][r]^{-1},[r][q p][r q p]^{-1}\right) z_{\mathrm{Gp}} \\
& =([r],[q]) z_{\mathrm{Gp}}-r \cdot\left([q],[p][r q p]^{-1}\right) z_{\mathrm{Gp}}+\left([r q][p],[r q p]^{-1}\right) z_{\mathrm{Gp}} \\
& -r q \cdot\left([p],[r q p]^{-1}\right) z_{\mathrm{Gp}}+([r q],[p]) z_{\mathrm{Gp}}+r \cdot\left([q, p],[q p][r q p]^{-1}\right) z_{\mathrm{Gp}} \\
& -\left([r],[q p][r q p]^{-1}\right) z_{\mathrm{Gp}} \\
& =([r],[q]) z_{\mathrm{Gp}}-r \cdot\left([q][p],[r q p]^{-1}\right) z_{\mathrm{Gp}}-r \cdot([q],[p]) z_{\mathrm{Gp}} \\
& +\left([r q][p],[r q p]^{-1}\right) z_{\mathrm{Gp}}+([r q],[p]) z_{\mathrm{Gp}}+r \cdot\left([q, p][q p],[r q p]^{-1}\right) z_{\mathrm{Gp}} \\
& -r \cdot\left([q p],[r q p]^{-1}\right) z_{\mathrm{Gp}}+r \cdot([q, p],[q p]) z_{\mathrm{Gp}}-\left([r][q p],[r q p]^{-1}\right) z_{\mathrm{Gp}} \\
& +r \cdot\left([q p],[r q p]^{-1}\right) z_{\mathrm{Gp}}-([r],[q p]) z_{\mathrm{Gp}} \\
& =([r],[q]) z_{\mathrm{Gp}}-r \cdot([q],[p]) z_{\mathrm{Gp}}+\left([r q, p][r q p],[r q p]^{-1}\right) z_{\mathrm{Gp}} \\
& +([r q],[p]) z_{\mathrm{Gp}}-\left([r, q p][r q p],[r q p]^{-1}\right) z_{\mathrm{Gp}}-([r],[q p]) z_{\mathrm{Gp}} \\
& =([r],[q]) z_{\mathrm{Gp}}-r \cdot([q],[p]) z_{\mathrm{Gp}}+([r q, p], 1) z_{\mathrm{Gp}}-([r q, p],[r q p]) z_{\mathrm{Gp}} \\
& +\left([r q p],[r q p]^{-1}\right) z_{\mathrm{Gp}}+([r q],[p]) z_{\mathrm{Gp}}-([r, q p], 1) z_{\mathrm{Gp}} \\
& +([r, q p],[r q p]) z_{\mathrm{Gp}}-\left([r q p],[r q p]^{-1}\right) z_{\mathrm{Gp}}-([r],[q p]) z_{\mathrm{Gp}}
\end{aligned}
$$




$$
\begin{aligned}
= & ([r],[q]) z_{\mathrm{Gp}}-([r],[q p]) z_{\mathrm{Gp}}+([r q],[p]) z_{\mathrm{Gp}}-r \cdot([q],[p]) z_{\mathrm{Gp}} \\
= & (r, q)\left(\left(s^{0} \times s^{0}\right) z_{\mathrm{Gp}}\right)-(r, q p)\left(\left(s^{0} \times s^{0}\right) z_{\mathrm{Gp}}\right)+(r q, p)\left(\left(s^{0} \times s^{0}\right) z_{\mathrm{Gp}}\right) \\
& \quad-r \cdot(q, p)\left(\left(s^{0} \times s^{0}\right) z_{\mathrm{Gp}}\right) \\
= & (r, q, p)\left(\left(\left(s^{0} \times s^{0}\right) z_{\mathrm{Gp}}\right) \partial\right)
\end{aligned}
$$

for $p, q, r \in \Pi_{0}$.

Conversely, we suppose given a pointed 2-cochain $z \in \mathrm{Ch}_{\mathrm{pt}}^{2}(E, M)$ that fulfills conditions (i) to (v). To show that $z$ is a 2-cocycle, we use the characterisation given in proposition $3.11(\mathrm{~b})$. First of all, we show that $z_{\mathrm{Gp}} \in \mathrm{Z}^{2}(\mathrm{Gp} E, M)$. Indeed, we have

$$
\begin{aligned}
& (k, h) \kappa(k h, g) \kappa((k, h g) \kappa)^{-1}\left({ }^{[\bar{k}]}((h, g) \kappa)\right)^{-1}[\bar{k}, \bar{h}, \bar{g}] \iota \\
& =((k, h) \kappa[\bar{k}, \bar{h}])((k h, g) \kappa[\bar{k} \bar{h}, \bar{g}])((k, h g) \kappa[\bar{k}, \bar{h} \bar{g}])^{-1}\left({ }^{[\bar{k}}((h, g) \kappa[\bar{h}, \bar{g}])\right)^{-1} \\
& =\left({ }^{[\bar{k}]}\left(\left[h[\bar{h}]^{-1}\right]^{-1}\right)\left[k[\bar{k}]^{-1}\right]^{-1}\left[k h[\overline{k h}]^{-1}\right]\right) \\
& \left.\left({ }^{[\overline{k h}}\right]\left(\left[g[\bar{g}]^{-1}\right]^{-1}\right)\left[k h[\overline{k h}]^{-1}\right]^{-1}\left[k h g[\overline{k h g}]^{-1}\right]\right) \\
& \left.\left({ }^{[\bar{k}}\right]\left(\left[h g[\overline{h g}]^{-1}\right]^{-1}\right)\left[k[\bar{k}]^{-1}\right]^{-1}\left[k h g[\overline{k h g}]^{-1}\right]\right)^{-1} \\
& \left([\bar{k}][\bar{h}]\left(\left[g[\bar{g}]^{-1}\right]^{-1}\right)^{[\bar{k}]}\left[h[\bar{h}]^{-1}\right]^{-1[\bar{k}]}\left[h g[\overline{h g}]^{-1}\right]\right)^{-1} \\
& \left.={ }^{[\bar{k}}\right]\left(\left[h[\bar{h}]^{-1}\right]^{-1}\right)\left[k[\bar{k}]^{-1}\right]^{-1}\left[k h[\overline{k h}]^{-1}\right]^{[\overline{k h}]}\left(\left[g[\bar{g}]^{-1}\right]^{-1}\right)\left[k h[\overline{k h}]^{-1}\right]^{-1} \\
& {\left[k h g[\overline{k h g}]^{-1}\right]\left[k h g[\overline{k h g}]^{-1}\right]^{-1}\left[k[\bar{k}]^{-1}\right]^{[\bar{k}]}\left[h g[\overline{h g}]^{-1}\right]\left({ }^{[\bar{k}}\left[h g[\overline{h g}]^{-1}\right]\right)^{-1}} \\
& \left.{ }^{[\bar{k}}\right]\left[h[\bar{h}]^{-1}\right]^{[\bar{k}][\bar{h}]}\left[g[\bar{g}]^{-1}\right] \\
& ={ }^{[\bar{k}]}\left(\left[h[\bar{h}]^{-1}\right]^{-1}\right)\left[k[\bar{k}]^{-1}\right]^{-1}\left[k h[\overline{k h}]^{-1}\right]^{[\overline{k h}]}\left(\left[g[\bar{g}]^{-1}\right]^{-1}\right)\left[k h[\overline{k h}]^{-1}\right]^{-1}\left[k[\bar{k}]^{-1}\right] \\
& { }^{[\bar{k}}\left[h[\bar{h}]^{-1}\right]^{[\bar{k}][\bar{h}]}\left[g[\bar{g}]^{-1}\right] \\
& ={ }^{[\bar{k}]}\left(\left[h[\bar{h}]^{-1}\right]^{-1}\right)^{\left[k[\bar{k}]^{-1}\right]^{-1}\left[k h[\overline{k h}]^{-1}\right][\overline{k h}]}\left(\left[g[\bar{g}]^{-1}\right]^{-1}\right)^{[\bar{k}]}\left[h[\bar{h}]^{-1}\right]^{[\bar{k}][\bar{h}]}\left[g[\bar{g}]^{-1}\right] \\
& ={ }^{[\bar{k}]}\left(\left[h[\bar{h}]^{-1}\right]^{-1}\right)^{[\bar{k}] h}\left(\left[g[\bar{g}]^{-1}\right]^{-1}\right)^{[\bar{k}]}\left[h[\bar{h}]^{-1}\right]^{[\bar{k}][\bar{h}]}\left[g[\bar{g}]^{-1}\right] \\
& ={ }^{[\bar{k}}\left(\left[h[\bar{h}]^{-1}\right]^{-1 h}\left(\left[g[\bar{g}]^{-1}\right]^{-1}\right)\left[h[\bar{h}]^{-1}\right]^{[\bar{h}]}\left[g[\bar{g}]^{-1}\right]\right) \\
& \left.={ }^{[\bar{k}}\right]\left(\left[h[\bar{h}]^{-1}\right]^{-1} h\left(\left[g[\bar{g}]^{-1}\right]^{-1}\right)^{[\bar{h}]}\left[g[\bar{g}]^{-1}\right]\right) \\
& ={ }^{[\bar{k}]}\left({ }^{[\bar{h}}\left(\left[g[\bar{g}]^{-1}\right]^{-1}\right)^{[\bar{h}]}\left[g[\bar{g}]^{-1}\right]\right)=1
\end{aligned}
$$

and hence

$$
\begin{aligned}
(k, h, g)\left(z_{\mathrm{Gp}} \partial\right)=(k, h) z_{\mathrm{Gp}}-(k, h g) z_{\mathrm{Gp}}+(k h, g) z_{\mathrm{Gp}}-\bar{k} \cdot(h, g) z_{\mathrm{Gp}} \\
=((k, h) \kappa) z_{\mathrm{Mp}}+([\bar{k}],[\bar{h}]) z_{\mathrm{Gp}}-((k, h g) \kappa) z_{\mathrm{Mp}}-([\bar{k}],[\overline{h g}]) z_{\mathrm{Gp}} \\
\quad+((k h, g) \kappa) z_{\mathrm{Mp}}+([\overline{k h}],[\bar{g}]) z_{\mathrm{Gp}}-\bar{k} \cdot((h, g) \kappa) z_{\mathrm{Mp}}-\bar{k} \cdot([\bar{h}],[\bar{g}]) z_{\mathrm{Gp}} \\
=\left((k, h) \kappa(k h, g) \kappa((k, h g) \kappa)^{-1}[\bar{k}]\left(((h, g) \kappa)^{-1}\right)\right) z_{\mathrm{Mp}} \\
\quad+(\bar{k}, \bar{h}, \bar{g})\left(\left(\left(s^{0} \times s^{0}\right) z_{\mathrm{Gp}}\right) \partial\right) \\
=\left((k, h) \kappa(k h, g) \kappa((k, h g) \kappa)^{-1}\left({ }^{[\bar{k}]}((h, g) \kappa)\right)^{-1}[\bar{k}, \bar{h}, \bar{g}] \iota\right) z_{\mathrm{Mp}}=0
\end{aligned}
$$


for $g, h, k \in \mathrm{Gp} E$, that is, $z_{\mathrm{Gp}} \in \mathrm{Z}_{\mathrm{pt}}^{2}(\mathrm{Gp} E, M)$. Moreover, we have

$$
\begin{aligned}
(n m) & z_{\mathrm{Mp}}-(n) z_{\mathrm{Mp}}-(m) z_{\mathrm{Mp}}+(n, m) z_{\mathrm{Gp}} \\
& =\left(n m[n m]^{-1}\right) z_{\mathrm{Mp}}-\left(n[n]^{-1}\right) z_{\mathrm{Mp}}-\left(m[m]^{-1}\right) z_{\mathrm{Mp}}+((n, m) \kappa) z_{\mathrm{Mp}} \\
& =\left(\left(n m[n m]^{-1}\right)\left(n[n]^{-1}\right)^{-1}\left(m[m]^{-1}\right)^{-1}(n, m) \kappa\right) z_{\mathrm{Mp}} \\
& =\left(\left(n[n]^{-1}\right)^{-1} n\left(m[m]^{-1}\right)^{-1} m(n, m) \kappa[n m]^{-1}\right) z_{\mathrm{Mp}} \\
& =\left([n] n^{-1} n[m] m^{-1} m[n]^{-1} n\left([m]^{-1}\right)[n m][n m]^{-1}\right) z_{\mathrm{Mp}} \\
& =\left([n][m][n]^{-1 n}\left([m]^{-1}\right)\right) z_{\mathrm{Mp}}=\left({ }^{[n]}[m]^{n}\left([m]^{-1}\right)\right) z_{\mathrm{Mp}}=0
\end{aligned}
$$

for $m, n \in \operatorname{Mp} E$ and

$$
\begin{aligned}
&\left({ }^{g} m\right) z_{\mathrm{Mp}}-\bar{g} \cdot(m) z_{\mathrm{Mp}}-\left({ }^{g} m, g\right) z_{\mathrm{Gp}}+(g, m) z_{\mathrm{Gp}} \\
&=\left({ }^{g} m\left[{ }^{g} m\right]^{-1}\right) z_{\mathrm{Mp}}-\bar{g} \cdot\left(m[m]^{-1}\right) z_{\mathrm{Mp}}-\left(\left({ }^{g} m, g\right) \kappa\right) z_{\mathrm{Mp}}+((g, m) \kappa) z_{\mathrm{Mp}} \\
&=\left(\left({ }^{g} m\left[{ }^{g} m\right]^{-1}\right){ }^{g}\left(\left(m[m]^{-1}\right)^{-1}\right)\left(\left({ }^{g} m, g\right) \kappa\right)^{-1}(g, m) \kappa\right) z_{\mathrm{Mp}} \\
&=\left(\left({ }^{g}[m]^{g}\left(m^{-1}\right)\right)\left({ }^{g} m\left[{ }^{g} m\right]^{-1}\right)\left(\left({ }^{g} m, g\right) \kappa\right){ }^{-1}(g, m) \kappa\right) z_{\mathrm{Mp}} \\
&=\left({ }^{g}[m](g, m) \kappa\left(\left({ }^{g} m, g\right) \kappa\right)^{-1}\left[{ }^{g} m\right]^{-1}\right) z_{\mathrm{Mp}} \\
&=\left({ } ^ { g } [ m ] ( [ g [ \overline { g } ] ^ { - 1 } ] ^ { - 1 } g ( [ m ] ^ { - 1 } ) [ g m [ \overline { g } ] ^ { - 1 } ] ) \left(\left[{ }^{g} m\right]^{-1}{ }^{g} m\left(\left[g[\bar{g}]^{-1}\right]^{-1}\right)\right.\right. \\
& \\
&\left.\left.=\left({ }^{g} m g[\bar{g}]^{-1}\right]\right)^{-1}\left[{ }^{g} m\right]^{-1}\right) z_{\mathrm{Mp}} \\
&=\left({ }^{g}[m]\left[g[\bar{g}]^{-1}\right]^{-1} g\left([m]^{-1}\right)\left[g m[\bar{g}]^{-1}\right]\left[{ }^{g} m g[\bar{g}]^{-1}\right]^{-1}{ }^{g} m\left[g[\bar{g}]^{-1}\right]\left[{ }^{g} m\right]\right. \\
&=\left.\left.\quad\left[{ }^{g} m\right]^{-1}\right) z_{\mathrm{Mp}}[m]\left[g[\bar{g}]^{-1}\right]^{-1} g\left([m]^{-1}\right){ }^{g} m\left[g[\bar{g}]^{-1}\right]\right) z_{\mathrm{Mp}} \\
&=\left({ }^{g}[m]\left(\left[g[\bar{g}]^{-1}\right]^{-1}\right){ }^{g} m\left[g[\bar{g}]^{-1}\right]\right) z_{\mathrm{Mp}}=0
\end{aligned}
$$

for $m \in \operatorname{Mp} E$ and $g \in \operatorname{Gp} E$. Altogether, $z \in \mathrm{Z}_{\mathrm{pt}}^{2}(E, M)$. Finally, we have

$$
([m]) z_{\mathrm{Mp}}=\left([m][m]^{-1}\right) z_{\mathrm{Mp}}=0
$$

for $m \in \operatorname{Mp} E$ and

$$
\begin{aligned}
\left(g[\bar{g}]^{-1},[\bar{g}]\right) z_{\mathrm{Gp}} & =\left(\left(g[\bar{g}]^{-1},[\bar{g}]\right) \kappa\right) z_{\mathrm{Mp}}+(1,[\bar{g}]) z_{\mathrm{Gp}} \\
& =\left(\left[g[\bar{g}]^{-1}\right]^{-1}\left[g[\bar{g}]^{-1}\right]\right) z_{\mathrm{Mp}}=0
\end{aligned}
$$

for $g \in \operatorname{Gp} E$. Hence $z \in \mathrm{Z}_{\mathrm{st}}^{2}(E, M)$ by corollary $4.5(\mathrm{~b})$.

(b) We suppose given a standard 2-coboundary $b \in \mathrm{B}_{\mathrm{st}}^{2}(E, M)$ and we choose $c \in$ $\mathrm{Ch}_{\mathrm{pt}}^{1}(E, M)$ such that $b=c \partial$. Letting $c_{0} \in \mathrm{Ch}_{\mathrm{pt}}^{1}\left(\Pi_{0}, M\right)$ be defined by $(p) c_{0}:=$ $([p]) c$, proposition $4.4(\mathrm{~b})$ implies that $(m) b_{\mathrm{Mp}}=(m) b_{\mathrm{Mp}}^{\mathrm{st}}=0$ for $m \in \mathrm{Mp} E$ and $(h, g) b_{\mathrm{Gp}}=(h, g) b_{\mathrm{Gp}}^{\mathrm{st}}=(\bar{h}, \bar{g})\left(c_{0} \partial\right)$ for $g, h \in \mathrm{Gp} E$.

Conversely, let us suppose that $b_{\mathrm{Mp}}=0$ and suppose given a pointed 1-cochain $c_{0} \in \mathrm{Ch}_{\mathrm{pt}}^{1}\left(\Pi_{0}, M\right) \quad$ with $(h, g) b_{\mathrm{Gp}}=(\bar{h}, \bar{g})\left(c_{0} \partial\right)$ for $g, h \in \mathrm{Gp} E$. Defining $c \in \mathrm{Ch}_{\mathrm{pt}}^{1}(E, M)$ by $(g) c:=(\bar{g}) c_{0}$ for $g \in \mathrm{Gp} E$, we have

$$
(m)(c \partial)_{\mathrm{Mp}}=(m) c=(\bar{m}) c_{0}=0
$$


for $m \in \operatorname{Mp} E$ and

$$
\begin{aligned}
(h, g)(c \partial)_{\mathrm{Gp}} & =(h) c-(h g) c+\bar{h} \cdot(g) c=(\bar{h}) c_{0}-(\overline{h g}) c_{0}+\bar{h} \cdot(\bar{g}) c_{0} \\
& =(\bar{h}, \bar{g})\left(c_{0} \partial\right),
\end{aligned}
$$

that is, $c \partial=b$. Moreover, $([m]) b_{\mathrm{Mp}}=0$ for all $m \in \operatorname{Mp} E$ and $\left(g[\bar{g}]^{-1},[\bar{g}]\right) b_{\mathrm{Gp}}=$ $(1, \bar{g})\left(c_{0} \partial\right)=0$ for all $g \in \mathrm{Gp} E$. Hence $b \in \mathrm{Z}_{\mathrm{st}}^{2}(E, M) \cap \mathrm{B}_{\mathrm{pt}}^{2}(E, M)=\mathrm{B}_{\mathrm{st}}^{2}(E, M)$ by corollary $4.5(\mathrm{~b})$.

Definition 4.8 (cocycle, coboundary and cohomology group of a 3-cocycle). For a 3-cocycle $z^{3} \in \mathrm{Z}^{3}\left(\Pi_{0}, \Pi_{1}\right)$, we set

$$
\begin{aligned}
& \mathrm{Z}^{2}\left(\left(\Pi_{0}, \Pi_{1}, z^{3}\right), M\right):=\operatorname{Hom}_{\Pi_{0}}\left(\Pi_{1}, M\right)_{\left.\operatorname{Map}\left(z^{3}, M\right)\right|_{\operatorname{Hom}_{\Pi_{0}}\left(\Pi_{1}, M\right)}} \times{ }_{\partial} \mathrm{Ch}_{\mathrm{cpt}}^{2}\left(\Pi_{0}, M\right), \\
& \mathrm{B}^{2}\left(\left(\Pi_{0}, \Pi_{1}, z^{3}\right), M\right):=\{0\} \times \mathrm{B}_{\mathrm{cpt}}^{2}\left(\Pi_{0}, M\right) \text {, and } \\
& \mathrm{H}^{2}\left(\left(\Pi_{0}, \Pi_{1}, z^{3}\right), M\right):=\mathrm{Z}^{2}\left(\left(\Pi_{0}, \Pi_{1}, z^{3}\right), M\right) / \mathrm{B}^{2}\left(\left(\Pi_{0}, \Pi_{1}, z^{3}\right), M\right) .
\end{aligned}
$$

Corollary 4.9. We have group homomorphisms $\Phi_{1}: \mathrm{Z}_{\mathrm{st}}^{2}(E, M) \rightarrow \operatorname{Hom}_{\Pi_{0}}\left(\Pi_{1}, M\right)$ and $\Phi_{0}: \mathrm{Z}_{\mathrm{st}}^{2}(E, M) \rightarrow \mathrm{Ch}_{\mathrm{pt}}^{2}\left(\Pi_{0}, M\right)$ given by $(k)\left(z \Phi_{1}\right):=(k \iota) z_{\mathrm{Mp}}$ for $k \in \Pi_{1}$ and $(q, p)\left(z \Phi_{0}\right):=([q],[p]) z_{\mathrm{Gp}}$ for $p, q \in \Pi_{0}, z \in \mathrm{Z}_{\mathrm{st}}^{2}(E, M)$. These group homomorphisms fit into the following diagram, which is a pullback of abelian groups.

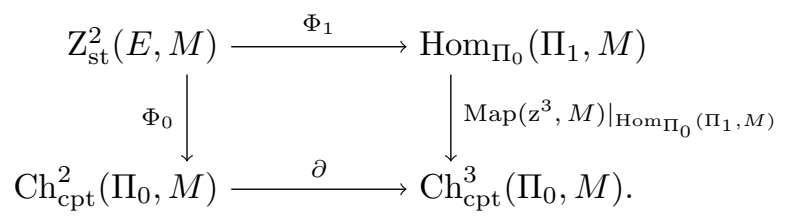

The induced isomorphism

$$
\Phi: \mathrm{Z}_{\mathrm{st}}^{2}(E, M) \rightarrow \mathrm{Z}^{2}\left(\left(\Pi_{0}, \Pi_{1}, \mathrm{z}^{3}\right), M\right), z \mapsto\left(z \Phi_{1}, z \Phi_{0}\right),
$$

whose inverse

$$
\Psi: \mathrm{Z}^{2}\left(\left(\Pi_{0}, \Pi_{1}, \mathrm{z}^{3}\right), M\right) \rightarrow \mathrm{Z}_{\mathrm{st}}^{2}(E, M)
$$

is given by $(m, h, g)\left(\left(z_{1}, c_{0}\right) \Psi\right)=\left(\left(m[m]^{-1}((m, h) \kappa)^{-1}(h, g) \kappa\right)\left(\left.\iota\right|^{\operatorname{Im} \iota}\right)^{-1}\right) z_{1}+(\bar{h}, \bar{g}) c_{0}$ for $m \in \mathrm{Mp} E, g, h \in \mathrm{Gp} E$, induces in turn isomorphisms

$$
\mathrm{B}_{\mathrm{st}}^{2}(E, M) \rightarrow \mathrm{B}^{2}\left(\left(\Pi_{0}, \Pi_{1}, \mathrm{z}^{3}\right), M\right) \quad \text { and } \quad \mathrm{H}_{\mathrm{st}}^{2}(E, M) \rightarrow \mathrm{H}^{2}\left(\left(\Pi_{0}, \Pi_{1}, \mathrm{z}^{3}\right), M\right) .
$$

In particular, we have

$$
\mathrm{H}^{2}(E, M) \cong \mathrm{H}^{2}\left(\left(\Pi_{0}, \Pi_{1}, \mathrm{z}^{3}\right), M\right) .
$$

Proof. By proposition 4.7(a)(iv) and (v), the group homomorphisms $\Phi_{0}$ and $\Phi_{1}$ are well-defined and the quadrangle commutes. To show that it is a pullback of abelian groups, we suppose given an arbitrary abelian group $T$ as well as group homomorphisms $\varphi_{0}: T \rightarrow \mathrm{Ch}_{\mathrm{cpt}}^{2}\left(\Pi_{0}, M\right)$ and $\varphi_{1}: T \rightarrow \operatorname{Hom}_{\Pi_{0}}\left(\Pi_{1}, M\right)$ such that $\left.\varphi_{1} \operatorname{Map}\left(\mathrm{z}^{3}, M\right)\right|_{\operatorname{Hom}_{\Pi_{0}}\left(\Pi_{1}, M\right)}=\varphi_{0} \partial$, that is, with $([r, q, p])\left(t \varphi_{1}\right)=(r, q, p)\left(\left(t \varphi_{0}\right) \partial\right)$ for all $p, q, r \in \Pi_{0}, t \in T$. For $t \in T$, we define a pointed 2 -cochain $t \varphi \in \mathrm{Ch}_{\mathrm{pt}}^{2}(E, M)$ by

$$
(m, h, g)(t \varphi):=\left(\left(m[m]^{-1}((m, h) \kappa)^{-1}(h, g) \kappa\right)\left(\left.\iota\right|^{\operatorname{Im} \iota}\right)^{-1}\right)\left(t \varphi_{1}\right)+(\bar{h}, \bar{g})\left(t \varphi_{0}\right)
$$

for $m \in \mathrm{Mp} E, g, h \in \mathrm{Gp} E$. We obtain $(m)(t \varphi)_{\mathrm{Mp}}=\left(\left(m[m]^{-1}\right)\left(\left.\iota\right|^{\operatorname{Im} \iota}\right)^{-1}\right)\left(t \varphi_{1}\right)$ for $m \in \mathrm{Mp} E$ and $(h, g)(t \varphi)_{\mathrm{Gp}}=\left((h, g) \kappa\left(\left.\iota\right|^{\operatorname{Im} \iota}\right)^{-1}\right)\left(t \varphi_{1}\right)+(\bar{h}, \bar{g})\left(t \varphi_{0}\right)$ for $g, h \in \mathrm{Gp} E$. 
To show that $t \varphi$ is a standard 2-cocycle, we verify the conditions in proposition 4.7(a). Indeed, using $\left[m[m]^{-1}\right]=([\bar{h}],[\bar{g}]) \kappa=(1, h) \kappa=1$ for $m \in \mathrm{Mp} E, g, h \in \mathrm{Gp} E$, we have

$$
\begin{gathered}
(m, h, g)(t \varphi)=\left(\left(m[m]^{-1}((m, h) \kappa)^{-1}(h, g) \kappa\right)\left(\left.\iota\right|^{\operatorname{Im} \iota}\right)^{-1}\right)\left(t \varphi_{1}\right)+(\bar{h}, \bar{g})\left(t \varphi_{0}\right) \\
=\left(\left(m[m]^{-1}\right)\left(\left.\iota\right|^{\operatorname{Im} \iota}\right)^{-1}\right)\left(t \varphi_{1}\right)-\left((m, h) \kappa\left(\left.\iota\right|^{\operatorname{Im} \iota}\right)^{-1}\right)\left(t \varphi_{1}\right) \\
\quad+\left((h, g) \kappa\left(\left.\iota\right|^{\operatorname{Im} \iota}\right)^{-1}\right)\left(t \varphi_{1}\right)+(\bar{h}, \bar{g})\left(t \varphi_{0}\right) \\
=(m)(t \varphi)_{\mathrm{Mp}}-(m, h)(t \varphi)_{\mathrm{Gp}}+(h, g)(t \varphi)_{\mathrm{Gp}}
\end{gathered}
$$

since $t \varphi_{1}$ is componentwise pointed as well as

$$
(m)(t \varphi)_{\mathrm{Mp}}=\left(m[m]^{-1}\right)\left(t \varphi_{1}\right)=\left(m[m]^{-1}\right)(t \varphi)_{\mathrm{Mp}}
$$

and

$$
\begin{aligned}
(h, g)(t \varphi)_{\mathrm{Gp}} & =\left((h, g) \kappa\left(\left.\iota\right|^{\operatorname{Im} \iota}\right)^{-1}\right)\left(t \varphi_{1}\right)+(\bar{h}, \bar{g})\left(t \varphi_{0}\right) \\
& =((h, g) \kappa)(t \varphi)_{\mathrm{Mp}}+([\bar{h}],[\bar{g}])(t \varphi)_{\mathrm{Gp}}
\end{aligned}
$$

for $m \in \operatorname{Mp} E, g, h \in \mathrm{Gp} E$. Moreover, $\mathfrak{\iota}(t \varphi)_{\mathrm{Mp}}=t \varphi_{1} \in \operatorname{Hom}_{\Pi_{0}}\left(\Pi_{1}, M\right)$ and

$$
\begin{aligned}
([r, q, p])(t \varphi)_{\mathrm{Mp}} & =([r, q, p] \iota)\left(t \varphi_{1}\right)=(r, q, p)\left(\left(t \varphi_{0}\right) \partial\right) \\
& =(r, q, p)\left(\left(\left(s^{0} \times s^{0}\right)(t \varphi)_{\mathrm{Gp}}\right) \partial\right)
\end{aligned}
$$

for $p, q, r \in \Pi_{0}$. Altogether, $t \varphi \in \mathrm{Z}_{\mathrm{st}}^{2}(E, M)$ for all $t \in T$, and we have constructed a well-defined group homomorphism $\varphi: T \rightarrow \mathrm{Z}_{\mathrm{st}}^{2}(E, M)$. Finally, we have

$$
(k)\left((t \varphi) \Phi_{1}\right)=(k \iota)(t \varphi)_{\mathrm{Mp}}=(k)\left(t \varphi_{1}\right)
$$

for $k \in \Pi_{1}, t \in T$, and

$$
\begin{aligned}
(q, p)\left((t \varphi) \Phi_{0}\right) & =([q],[p])(t \varphi)_{\mathrm{Gp}}=\left(([q],[p]) \kappa\left(\left.\iota\right|^{\operatorname{Im} \iota}\right)^{-1}\right)\left(t \varphi_{1}\right)+(q, p)\left(t \varphi_{0}\right) \\
& =(q, p)\left(t \varphi_{0}\right)
\end{aligned}
$$

for $p, q \in \Pi_{0}, t \in T$, that is, $\varphi \Phi_{1}=\varphi_{1}$ and $\varphi \Phi_{0}=\varphi_{0}$.

Conversely, given an arbitrary group homomorphism $\varphi: T \rightarrow \mathrm{Z}_{\mathrm{st}}^{2}(E, M)$ with $\varphi \Phi_{1}=\varphi_{1}$ and $\varphi \Phi_{0}=\varphi_{0}$, we necessarily have

$$
\begin{aligned}
(m)(t \varphi)_{\mathrm{Mp}} & =\left(m[m]^{-1}\right)(t \varphi)_{\mathrm{Mp}}=\left(\left(m[m]^{-1}\right)\left(\left.\iota\right|^{\operatorname{Im} \iota}\right)^{-1}\right)\left(t \varphi \Phi_{1}\right) \\
& =\left(\left(m[m]^{-1}\right)\left(\left.\iota\right|^{\operatorname{Im} \iota}\right)^{-1}\right)\left(t \varphi_{1}\right)
\end{aligned}
$$

for $m \in \operatorname{Mp} E$, and

$$
\begin{aligned}
(h, g)(t \varphi)_{\mathrm{Gp}} & =((h, g) \kappa)(t \varphi)_{\mathrm{Mp}}+([\bar{h}],[\bar{g}])(t \varphi)_{\mathrm{Gp}} \\
& =((h, g) \kappa)(t \varphi)_{\mathrm{Mp}}+(\bar{h}, \bar{g})\left(t \varphi \Phi_{0}\right) \\
& =\left((h, g) \kappa\left(\iota^{\mid \mathrm{Im} \iota}\right)^{-1}\right)\left(t \varphi_{1}\right)+(\bar{h}, \bar{g})\left(t \varphi_{0}\right)
\end{aligned}
$$

for $g, h \in \mathrm{Gp} E$. This shows the uniqueness of the induced group homomorphism. Altogether, the diagram under consideration is a pullback of abelian groups.

Our next step is to show that the induced isomorphism

$$
\Phi: \mathrm{Z}_{\mathrm{st}}^{2}(E, M) \rightarrow \mathrm{Z}^{2}\left(\left(\Pi_{0}, \Pi_{1}, \mathrm{z}^{3}\right), M\right)
$$

restricts to an isomorphism $\mathrm{B}_{\mathrm{st}}^{2}(E, M) \rightarrow \mathrm{B}^{2}\left(\left(\Pi_{0}, \Pi_{1}, \mathrm{z}^{3}\right), M\right)$. Given a standard 2coboundary $b \in \mathrm{B}_{\mathrm{st}}^{2}(E, M)$, proposition $4.7(\mathrm{~b})$ states that $b_{\mathrm{Mp}}=0$ and that there 
exists a pointed 1-cochain $c_{0} \in \mathrm{Ch}_{\mathrm{pt}}^{1}\left(\Pi_{0}, M\right)$ with $(h, g) b_{\mathrm{Gp}}=(\bar{h}, \bar{g})\left(c_{0} \partial\right)$ for $g, h \in$ Gp $E$. In particular, $b \Phi_{1}=0$ and

$$
(q, p)\left(b \Phi_{0}\right)=([q],[p]) b_{\mathrm{Gp}}=(q, p)\left(c_{0} \partial\right)
$$

for $p, q \in \Pi_{0}$ and hence $b \Phi_{0} \in \mathrm{B}^{2}\left(\Pi_{0}, M\right)$. Conversely, we suppose given a standard 2-cocycle $b \in \mathrm{Z}_{\mathrm{st}}^{2}(E, M)$ with $b \Phi_{1}=0$ and $b \Phi_{0} \in \mathrm{B}_{\mathrm{pt}}^{2}\left(\Pi_{0}, M\right)$, that is, there exists a pointed 1-cochain $c_{0} \in \mathrm{Ch}_{\mathrm{pt}}^{1}\left(\Pi_{0}, M\right)$ with $b \Phi_{0}=c_{0} \partial$. Then

$$
(m) b_{\mathrm{Mp}}=\left(m[m]^{-1}\right) b_{\mathrm{Mp}}=\left(\left(m[m]^{-1}\right)\left(\iota^{\mathrm{Im} \iota}\right)^{-1}\right)\left(b \Phi_{1}\right)=0
$$

for all $m \in \operatorname{Mp} E$ and

$$
(h, g) b_{\mathrm{Gp}}=((h, g) \kappa) b_{\mathrm{Mp}}+([\bar{h}],[\bar{g}]) b_{\mathrm{Gp}}=(\bar{h}, \bar{g})\left(b \Phi_{0}\right)=(\bar{h}, \bar{g})\left(c_{0} \partial\right)
$$

for all $g, h \in \operatorname{Gp} E$. Hence $b$ is a standard 2-coboundary by proposition 4.7(b).

Altogether, $\Phi$ restricts to an isomorphism $\mathrm{B}_{\mathrm{st}}^{2}(E, M) \rightarrow \mathrm{B}^{2}\left(\left(\Pi_{0}, \Pi_{1}, \mathrm{z}^{3}\right), M\right)$ and hence induces also an isomorphism $\mathrm{H}_{\mathrm{st}}^{2}(E, M) \rightarrow \mathrm{H}^{2}\left(\left(\Pi_{0}, \Pi_{1}, \mathrm{z}^{3}\right), M\right)$. Moreover, corollary $4.5(\mathrm{c})$ implies that

$$
\mathrm{H}^{2}(E, M) \cong \mathrm{H}_{\mathrm{st}}^{2}(E, M) \cong \mathrm{H}^{2}\left(\left(\Pi_{0}, \Pi_{1}, \mathrm{z}^{3}\right), M\right) .
$$

Corollary 4.10. For $z^{3}, \tilde{z}^{3} \in \mathrm{Z}_{\mathrm{cpt}}^{3}\left(\Pi_{0}, \Pi_{1}\right)$ with $z^{3} \mathrm{~B}_{\mathrm{cpt}}^{3}\left(\Pi_{0}, \Pi_{1}\right)=\tilde{z}^{3} \mathrm{~B}_{\mathrm{cpt}}^{3}\left(\Pi_{0}, \Pi_{1}\right)$, we have

$$
\mathrm{H}^{2}\left(\left(\Pi_{0}, \Pi_{1}, z^{3}\right), M\right) \cong \mathrm{H}^{2}\left(\left(\Pi_{0}, \Pi_{1}, \tilde{z}^{3}\right), M\right) .
$$

Proof. We suppose given 3-cocycles $z^{3}, \tilde{z}^{3} \in \mathrm{Z}_{\mathrm{cpt}}^{3}\left(\Pi_{0}, \Pi_{1}\right)$ with $z^{3} \mathrm{~B}_{\mathrm{cpt}}^{3}\left(\Pi_{0}, \Pi_{1}\right)=$ $\tilde{z}^{3} \mathrm{~B}_{\mathrm{cpt}}^{3}\left(\Pi_{0}, \Pi_{1}\right)$. By construction of the standard extension $\mathrm{E}\left(z^{3}\right)$, the 3 -cocycle of the standard extension $\mathrm{E}\left(z^{3}\right)$ with respect to the standard section system $\left(\mathrm{s}_{z^{3}}^{1}, \mathrm{~s}_{z^{3}}^{0}\right)$ is given by $\mathrm{z}_{\mathrm{E}\left(z^{3}\right),\left(\mathrm{s}_{z^{3}}^{1}, \mathrm{~s}_{z^{3}}^{0}\right)}=z^{3}$, cf. section 2.13. Moreover, by [31, prop. (6.5)] there exists a section system $\left(\tilde{s}^{1}, \mathrm{~s}_{z^{3}}^{0}\right)$ for $\mathrm{E}\left(z^{3}\right)$ such that $\mathrm{z}_{\mathrm{E}\left(z^{3}\right),\left(\tilde{s}^{1}, \mathrm{~s}_{z^{3}}^{0}\right)}=\tilde{z}^{3}$. Thus corollary 4.9 implies

$$
\mathrm{H}^{2}\left(\left(\Pi_{0}, \Pi_{1}, z^{3}\right), M\right) \cong \mathrm{H}^{2}\left(\mathrm{E}\left(z^{3}\right), M\right) \cong \mathrm{H}^{2}\left(\left(\Pi_{0}, \Pi_{1}, \tilde{z}^{3}\right), M\right) .
$$

We finish this section by a direct algebraic proof that extension equivalent crossed module extensions yield the same second cohomology group, as to be expected from a weak homotopy equivalence, cf. for example [31, rem. (4.5)].

Proposition 4.11. We suppose given crossed module extensions $E$ and $\tilde{E}$ of $\Pi_{0}$ with $\Pi_{1}$ and an extension equivalence $\varphi: E \rightarrow \tilde{E}$. Moreover, we suppose given a section system $\left(s^{1}, s^{0}\right)$ for $E$ and a section system $\left(\tilde{s}^{1}, \tilde{s}^{0}\right)$ for $\tilde{E}$ such that $\tilde{s}^{0}=s^{0}(\operatorname{Gp} \varphi)$ and $s^{1}(\operatorname{Mp} \varphi)=\left.(\operatorname{Gp} \varphi)\right|_{\operatorname{Im} \mu^{E}} ^{\operatorname{Im} \mu^{\tilde{E}}} \tilde{s}^{1} \cdot\left({ }^{7}\right)$

The induced group homomorphism $\mathrm{Z}^{2}(\varphi, M): \mathrm{Z}^{2}(\tilde{E}, M) \rightarrow \mathrm{Z}^{2}(E, M)$ restricts to an isomorphism $\mathrm{Z}_{\mathrm{st},\left(\tilde{s}^{1}, \tilde{s}^{0}\right)}^{2}(\tilde{E}, M) \rightarrow \mathrm{Z}_{\mathrm{st},\left(s^{1}, s^{0}\right)}^{2}(E, M)$, which induces in turn isomorphisms

$$
\mathrm{B}_{\mathrm{st},\left(\tilde{s}^{1}, \tilde{s}^{0}\right)}^{2}(\tilde{E}, M) \rightarrow \mathrm{B}_{\mathrm{st},\left(s^{1}, s^{0}\right)}^{2}(E, M) \quad \text { and } \quad \mathrm{H}_{\mathrm{st},\left(\tilde{s}^{1}, \tilde{s}^{0}\right)}^{2}(\tilde{E}, M) \rightarrow \mathrm{H}_{\mathrm{st},\left(s^{1}, s^{0}\right)}^{2}(E, M)
$$

\footnotetext{
${ }^{7}$ Such section systems exist, cf. for example [31, prop. (5.16)(b)].
} 
Proof. To show that $\mathrm{Z}^{2}(\varphi, M)$ restricts to a group homomorphism $\mathrm{Z}_{\mathrm{st}}^{2}(\tilde{E}, M) \rightarrow$ $\mathrm{Z}_{\mathrm{st}}^{2}(E, M)$, we have to show that $\tilde{z} \mathrm{Z}^{2}(\varphi, M) \in \mathrm{Z}_{\mathrm{st}}^{2}(E, M)$ for every given standard 2-cocycle $\tilde{z} \in \mathrm{Z}_{\mathrm{st}}^{2}(\tilde{E}, M)$. By corollary $4.5(\mathrm{~b})$, we have

$$
\left(\tilde{m} \tilde{s}^{1}\right) \tilde{z}_{\mathrm{Mp}}=\left(\tilde{g}\left(\tilde{g} \pi^{\tilde{E}} \tilde{s}^{0}\right)^{-1}, \tilde{g} \pi^{\tilde{E}} \tilde{s}^{0}\right) \tilde{z}_{\mathrm{Gp}}=0
$$

for all $\tilde{m} \in \operatorname{Mp} \tilde{E}, \tilde{g} \in \operatorname{Gp} \tilde{E}$. Since $s^{0}(\operatorname{Gp} \varphi)=\tilde{s}^{0}$ and $s^{1}(\operatorname{Mp} \varphi)=\left.(\operatorname{Gp} \varphi)\right|_{\operatorname{Im} \mu^{E}} ^{\operatorname{Im} \tilde{s}^{1}}$, it $^{\tilde{E}}$ follows that

$$
\left(m s^{1}\right)\left(\tilde{z} \mathrm{Z}^{2}(\varphi, M)\right)_{\mathrm{Mp}}=\left(m s^{1} \varphi\right) \tilde{z}_{\mathrm{Mp}}=\left(m \varphi \tilde{s}^{1}\right) \tilde{z}_{\mathrm{Mp}}=0
$$

for all $m \in \operatorname{Mp} E$ and

$$
\begin{aligned}
\left(g\left(g \pi^{E} s^{0}\right)^{-1}, g \pi^{E} s^{0}\right)\left(\tilde{z} \mathrm{Z}^{2}(\varphi, M)\right)_{\mathrm{Gp}} & =\left((g \varphi)\left(g \pi^{E} s^{0} \varphi\right)^{-1}, g \pi^{E} s^{0} \varphi\right) \tilde{z}_{\mathrm{Gp}} \\
& =\left((g \varphi)\left((g \varphi) \pi^{\tilde{E}} \tilde{s}^{0}\right)^{-1},(g \varphi) \pi^{\tilde{E}} \tilde{s}^{0}\right) \tilde{z}_{\mathrm{Gp}}=0
\end{aligned}
$$

for all $g \in \operatorname{Gp} E$, that is, $\tilde{z} \mathrm{Z}^{2}(\varphi, M) \in \mathrm{Z}_{\mathrm{st}}^{2}(E, M)$ by corollary $4.5(\mathrm{~b})$. Hence $\mathrm{Z}^{2}(\varphi, M)$ restricts to a well-defined group homomorphism

$$
\left.\mathrm{Z}^{2}(\varphi, M)\right|_{\mathrm{Z}_{\mathrm{st}}^{2}(\tilde{E}, M)} ^{\mathrm{Z}_{2}^{2}(E, M)}: \mathrm{Z}_{\mathrm{st}}^{2}(\tilde{E}, M) \rightarrow \mathrm{Z}_{\mathrm{st}}^{2}(E, M)
$$

Now, $[31$, prop. $(5.14)(\mathrm{c})]$ implies that $\mathrm{z}_{E,\left(s^{1}, s^{0}\right)}^{3}=\mathrm{z}_{\tilde{E},\left(\tilde{s}^{1}, \tilde{s}^{0}\right)}^{3}$. By corollary 4.9, we have isomorphisms

$$
\Phi: \mathrm{Z}_{\mathrm{st}}^{2}(E, M) \rightarrow \mathrm{Z}^{2}\left(\left(\Pi_{0}, \Pi_{1}, \mathrm{z}^{3}\right), M\right), z \mapsto\left(z \Phi_{1}, z \Phi_{0}\right)
$$

given by $(k)\left(z \Phi_{1}\right):=\left(k \iota^{E}\right) z_{\mathrm{Mp}}$ for $k \in \Pi_{1}$ and $(q, p)\left(z \Phi_{0}\right):=\left(q s^{0}, p s^{0}\right) z_{\mathrm{Gp}}$ for $p, q \in$ $\Pi_{0}, z \in \mathrm{Z}_{\mathrm{st}}^{2}(E, M)$, and

$$
\tilde{\Phi}: \mathrm{Z}_{\mathrm{st}}^{2}(\tilde{E}, M) \rightarrow \mathrm{Z}^{2}\left(\left(\Pi_{0}, \Pi_{1}, \mathrm{z}^{3}\right), M\right), \tilde{z} \mapsto\left(\tilde{z} \tilde{\Phi}_{1}, \tilde{z} \tilde{\Phi}_{0}\right)
$$

given by $(k)\left(\tilde{z} \tilde{\Phi}_{1}\right):=\left(k \iota^{\tilde{E}}\right) \tilde{z}_{\mathrm{Mp}}$ for $k \in \Pi_{1}$ and $(q, p)\left(\tilde{z} \tilde{\Phi}_{0}\right):=\left(q \tilde{s}^{0}, p \tilde{s}^{0}\right) \tilde{z}_{\mathrm{Gp}}$ for $p, q \in$ $\Pi_{0}, \tilde{z} \in \mathrm{Z}_{\mathrm{st}}^{2}(\tilde{E}, M)$. To show that $\left.\mathrm{Z}^{2}(\varphi, M)\right|_{\mathrm{Z}_{\mathrm{st}}^{2}(\tilde{E}, M)} ^{\mathrm{Z}_{\mathrm{s}}^{2}(E, M)}$ is an isomorphism, it suffices to verify that $\tilde{\Phi}=\left.\left(\mathrm{Z}^{2}(\varphi, M)\right)\right|_{\mathrm{Z}_{\mathrm{st}}^{2}(\tilde{E}, M)} ^{\mathrm{Z}_{\mathrm{E}}^{2}(E, M)} \Phi$. Indeed, given $\tilde{z} \in \mathrm{Z}_{\mathrm{st}}^{2}(\tilde{E}, M)$, we have

$$
k\left(\tilde{z} \mathrm{Z}^{2}(\varphi, M) \Phi_{1}\right)=\left(k \iota^{E}\right)\left(\tilde{z} \mathrm{Z}^{2}(\varphi, M)\right)_{\mathrm{Mp}}=\left(k \iota^{E} \varphi\right) \tilde{z}_{\mathrm{Mp}}=\left(k \iota^{\tilde{E}}\right) \tilde{z}_{\mathrm{Mp}}=k\left(\tilde{z} \tilde{\Phi}_{1}\right)
$$

for all $k \in \Pi_{1}$ and

$$
\begin{aligned}
(q, p)\left(\tilde{z} \mathrm{Z}^{2}(\varphi, M) \Phi_{0}\right) & =\left(q s^{0}, p s^{0}\right)\left(\tilde{z} \mathrm{Z}^{2}(\varphi, M)\right)_{\mathrm{Gp}}=\left(q s^{0} \varphi, p s^{0} \varphi\right) \tilde{z}_{\mathrm{Gp}} \\
& =\left(q \tilde{s}^{0}, p \tilde{s}^{0}\right) \tilde{z}_{\mathrm{Gp}}=(q, p)\left(\tilde{z} \tilde{\Phi}_{0}\right)
\end{aligned}
$$

for all $p, q \in \Pi_{0}$, that is, $\tilde{\Phi}=\left.\left(\mathrm{Z}^{2}(\varphi, M)\right)\right|_{\mathrm{Z}_{\mathrm{st}}^{2}(\tilde{E}, M)} ^{\mathrm{Z}_{\mathrm{st}}^{2}(E, M)} \Phi$.

Moreover, the induced group homomorphism $\mathrm{B}^{2}(\varphi, M)$ also restricts to a welldefined group homomorphism

$$
\left.\mathrm{B}^{2}(\varphi, M)\right|_{\mathrm{B}_{\mathrm{st}}^{2}(\tilde{E}, M)} ^{\mathrm{B}_{\mathrm{s}}^{2}(E, M)}: \mathrm{B}_{\mathrm{st}}^{2}(\tilde{E}, M) \rightarrow \mathrm{B}_{\mathrm{st}}^{2}(E, M)
$$

cf. definition $4.2(\mathrm{~b})$, which is an isomorphism since

$$
\left.\tilde{\Phi}\right|_{\mathrm{B}_{\mathrm{st}}^{2}(\tilde{E}, M)} ^{\mathrm{B}_{\mathrm{EM}}^{2}\left(\left(\Pi_{0}, \Pi_{1}, \mathrm{z}^{3}\right), M\right)}=\left(\left.\mathrm{B}^{2}(\varphi, M)\right|_{\mathrm{B}_{\mathrm{st}}^{2}(\tilde{E}, M)} ^{\mathrm{B}_{2}^{2}(E, M)}\right)\left(\left.\Phi\right|_{\mathrm{B}_{\mathrm{st}}^{2}(E, M)} ^{\mathrm{B}_{\mathrm{EM}}^{2}\left(\left(\Pi_{0}, \Pi_{1}, \mathrm{z}^{3}\right), M\right)}\right)
$$


and since $\left.\Phi\right|_{\mathrm{B}_{\mathrm{st}}^{2}(E, M)} ^{\mathrm{B}_{\mathrm{EM}}^{2}\left(\left(\Pi_{0}, \Pi_{1}, \mathrm{z}^{3}\right), M\right)}$ and $\left.\tilde{\Phi}\right|_{\mathrm{B}_{\mathrm{st}}^{2}(\tilde{E}, M)} ^{\mathrm{B}_{\mathrm{EM}}^{2}\left(\left(\Pi_{0}, \Pi_{1}, \mathrm{z}^{3}\right), M\right)}$ are isomorphisms by corollary 4.9 .

Finally, it follows that we get an induced isomorphism

$$
\mathrm{H}_{\mathrm{st}}^{2}(\tilde{E}, M) \rightarrow \mathrm{H}_{\mathrm{st}}^{2}(E, M) \text {. }
$$

\section{Second Eilenberg-Mac Lane cohomology group}

Until now, we have worked with crossed module extensions. Since every crossed module gives rise to a canonical crossed module extension, we can now formulate EILENBERGS and MAC LANEs theorem in the context of crossed modules and simplicial groups.

Definition 5.1 (first Postnikov invariant).

(a) Given a crossed module $V$, the cohomology class associated to the canonical extension

$$
\pi_{1}(V) \stackrel{\text { inc }}{\longrightarrow} \mathrm{Mp} V \stackrel{\mu}{\longrightarrow} \mathrm{Gp} V \stackrel{\text { quo }}{\longrightarrow} \pi_{0}(V)
$$

will be denoted by $\mathrm{k}_{V}^{3}:=\operatorname{cl}(V) \in \mathrm{H}_{\mathrm{cpt}}^{3}\left(\pi_{0}(V), \pi_{1}(V)\right)$ and is called the (first) Postnikov invariant of $V$.

(b) Given a simplicial group $G$, we call $\mathrm{k}_{G}^{3}:=\operatorname{cl}\left(\operatorname{Trunc}^{1} G\right) \in \mathrm{H}_{\text {cpt }}^{3}\left(\pi_{0}(G), \pi_{1}(G)\right)$ the first Postnikov invariant of $G$.

Definition 5.2 (second Eilenberg-Mac Lane cohomology group, cf. [12, sec. 3]).

(a) We suppose given a crossed module $V$ and a componentwise pointed 3-cocycle $z^{3} \in \mathrm{Z}_{\mathrm{cpt}}^{3}\left(\pi_{0}(V), \pi_{1}(V)\right)$ with $\mathrm{k}_{V}^{3}=z^{3} \mathrm{~B}_{\mathrm{cpt}}^{3}\left(\pi_{0}(V), \pi_{1}(V)\right)$. The second Eilenberg-Mac Lane cohomology group of $V$ with respect to $z^{3}$ and with coefficients in $M$ is defined by

$$
\mathrm{H}_{\mathrm{EM}, z^{3}}^{2}(V, M):=\mathrm{H}^{2}\left(\left(\pi_{0}(V), \pi_{1}(V), z^{3}\right), M\right) .
$$

(b) We suppose given a simplicial group $G$ and a componentwise pointed 3-cocycle $z^{3} \in \mathrm{Z}_{\mathrm{cpt}}^{3}\left(\pi_{0}(G), \pi_{1}(G)\right)$ with $\mathrm{k}_{G}^{3}=z^{3} \mathrm{~B}_{\mathrm{cpt}}^{3}\left(\pi_{0}(G), \pi_{1}(G)\right)$. The second EilenbergMac Lane cohomology group of $G$ with respect to $z^{3}$ and with coefficients in $M$ is defined by

$$
\mathrm{H}_{\mathrm{EM}, z^{3}}^{2}(G, M):=\mathrm{H}^{2}\left(\left(\pi_{0}(G), \pi_{1}(G), z^{3}\right), M\right) .
$$

We have already seen that the isomorphism class of the second Eilenberg-Mac Lane cohomology group of a crossed module does not depend on the choice of a specific 3 -cocycle in its associated cohomology class:

Remark 5.3. Given a crossed module $V$ and componentwise pointed 3-cocycles $z^{3}$, $\tilde{z}^{3} \in \mathrm{Z}_{\mathrm{cpt}}^{3}\left(\pi_{0}(V), \pi_{1}(V)\right) \quad$ with $\mathrm{k}_{V}^{3}=z^{3} \mathrm{~B}_{\mathrm{cpt}}^{3}\left(\pi_{0}(V), \pi_{1}(V)\right)=\tilde{z}^{3} \mathrm{~B}_{\mathrm{cpt}}^{3}\left(\pi_{0}(V), \pi_{1}(V)\right)$, we have

$$
\mathrm{H}_{\mathrm{EM}, z^{3}}^{2}(V, M) \cong \mathrm{H}_{\mathrm{EM}, \tilde{z}^{3}}^{2}(V, M) .
$$

Proof. This follows from corollary 4.10. 
Theorem 5.4 (cf. [12, th. 2]).

(a) Given a crossed module $V$, an abelian $\pi_{0}(V)$-module $M$ and a componentwise pointed 3-cocycle $z^{3} \in \mathrm{Z}_{\mathrm{cpt}}^{3}\left(\pi_{0}(V), \pi_{1}(V)\right)$ with $\mathrm{k}_{V}^{3}=z^{3} \mathrm{~B}_{\mathrm{cpt}}^{3}\left(\pi_{0}(V), \pi_{1}(V)\right)$, we have

$$
\mathrm{H}^{2}(V, M) \cong \mathrm{H}_{\mathrm{EM}, z^{3}}^{2}(V, M) .
$$

(b) Given a simplicial group $G$, an abelian $\pi_{0}(G)$-module $M$ and a componentwise pointed 3 -cocycle $z^{3} \in \mathrm{Z}_{\mathrm{cpt}}^{3}\left(\pi_{0}(G), \pi_{1}(G)\right)$ with $\mathrm{k}_{G}^{3}=z^{3} \mathrm{~B}_{\mathrm{cpt}}^{3}\left(\pi_{0}(G), \pi_{1}(G)\right)$, we have

$$
\mathrm{H}^{2}(G, M) \cong \mathrm{H}_{\mathrm{EM}, z^{3}}^{2}(G, M) .
$$

Proof.

(a) This follows from corollary 4.9 and remark 5.3.

(b) Applying proposition 3.13 and (a), we obtain

$$
\mathrm{H}^{2}(G, M) \cong \mathrm{H}^{2}\left(\operatorname{Trunc}^{1} G, M\right) \cong \mathrm{H}_{\mathrm{EM}, z^{3}}^{2}\left(\operatorname{Trunc}^{1} G, M\right)=\mathrm{H}_{\mathrm{EM}, z^{3}}^{2}(G, M) .
$$

Corollary 5.5 (cf. [12, sec. 4]).

(a) We suppose given a simplicial group $G$ and an abelian $\pi_{0}(G)$-module $M$.

(i) If $\mathrm{k}_{G}^{3}=1$, then

$$
\mathrm{H}^{2}(G, M) \cong \operatorname{Hom}_{\pi_{0}(G)}\left(\pi_{1}(G), M\right) \oplus \mathrm{H}^{2}\left(\pi_{0}(G), M\right) .
$$

(ii) If $\operatorname{Hom}_{\pi_{0}(G)}\left(\pi_{1}(G), M\right)=\{0\}$, then

$$
\mathrm{H}^{2}(G, M) \cong \mathrm{H}^{2}\left(\pi_{0}(G), M\right) .
$$

(b) We suppose given a crossed module $V$ and an abelian $\pi_{0}(V)$-module $M$.

(i) If $\mathrm{k}_{V}^{3}=1$, then

$$
\mathrm{H}^{2}(V, M) \cong \operatorname{Hom}_{\pi_{0}(V)}\left(\pi_{1}(V), M\right) \oplus \mathrm{H}^{2}\left(\pi_{0}(V), M\right) .
$$

(ii) If $\operatorname{Hom}_{\pi_{0}(V)}\left(\pi_{1}(V), M\right)=\{0\}$, then

$$
\mathrm{H}^{2}(V, M) \cong \mathrm{H}^{2}\left(\pi_{0}(V), M\right) .
$$

Proof.

(a) (i) If $\mathrm{k}_{G}^{3}=1$, then we have $\mathrm{Z}^{2}\left(\left(\pi_{0}(G), \pi_{1}(G), 1\right), M\right)=\operatorname{Hom}_{\pi_{0}(G)}\left(\pi_{1}(G), M\right) \times$ $\mathrm{Z}_{\mathrm{cpt}}^{2}\left(\pi_{0}(G), M\right)$ and hence

$$
\begin{aligned}
\mathrm{H}^{2}(G, M) & \left.\cong \mathrm{H}_{\mathrm{EM}, 1}^{2}(G, M)=\mathrm{H}^{2}\left(\pi_{0}(G), \pi_{1}(G), 1\right), M\right) \\
& \cong \operatorname{Hom}_{\pi_{0}(G)}\left(\pi_{1}(G), M\right) \times \mathrm{H}_{\mathrm{cpt}}^{2}\left(\pi_{0}(G), M\right) \\
& \cong \operatorname{Hom}_{\pi_{0}(G)}\left(\pi_{1}(G), M\right) \oplus \mathrm{H}^{2}\left(\pi_{0}(G), M\right)
\end{aligned}
$$

by theorem 5.4 .

(ii) If $\operatorname{Hom}_{\pi_{0}(G)}\left(\pi_{1}(G), M\right)=\{0\}$, then we get

$$
\begin{aligned}
\mathrm{H}^{2}(G, M) & \left.\cong \mathrm{H}_{\mathrm{EM}, z^{3}}^{2}(G, M)=\mathrm{H}^{2}\left(\pi_{0}(G), \pi_{1}(G), z^{3}\right), M\right) \\
& \cong \mathrm{H}_{\mathrm{cpt}}^{2}\left(\pi_{0}(G), M\right) \cong \mathrm{H}^{2}\left(\pi_{0}(G), M\right),
\end{aligned}
$$

where $z^{3} \in \mathrm{Z}_{\mathrm{cpt}}^{3}\left(\pi_{0}(G), \pi_{1}(G)\right)$ with $\mathrm{k}_{G}^{3}=z^{3} \mathrm{~B}_{\mathrm{cpt}}^{3}\left(\pi_{0}(G), \pi_{1}(G)\right)$.

(b) This follows from (a) applied to the simplicial group $\operatorname{Cosk}_{1} V$. 
Question 5.6 (cf. [12, sec. 5]).

(a) We suppose given a crossed module $V$ and an abelian $\pi_{0}(V)$-module $M$. How can theorem 5.4 be generalised to obtain a description of $\mathrm{H}^{n}(V, M)$ for $n \geqslant 3$ in terms of $\pi_{0}(V), \pi_{1}(V)$ and $\mathrm{k}_{V}^{3}$ ? What about such descriptions for homology?

(b) We suppose given a simplicial group $G$ and an abelian $\pi_{0}(V)$-module $M$. How can theorem 5.4 be generalised to obtain a description of $\mathrm{H}^{n}(G, M)$ for $n \geqslant$ 3 in terms of homotopy groups and Postnikov invariants? What about such descriptions for homology?

Finally, we discuss some examples.

Example 5.7. We suppose given a group $\Pi_{0}$ and abelian $\Pi_{0}$-modules $\Pi_{1}$ and $M$. We let $E$ be the crossed module extension

$$
\Pi_{1} \stackrel{\mathrm{id}_{\Pi_{1}}}{\longrightarrow} \Pi_{1} \stackrel{\text { triv }}{\longrightarrow} \Pi_{0} \stackrel{\operatorname{id}_{\Pi_{0}}}{\longrightarrow} \Pi_{0} .
$$

Then we have

$$
\mathrm{H}^{2}(E, M) \cong \operatorname{Hom}_{\Pi_{0}}\left(\Pi_{1}, M\right) \oplus \mathrm{H}^{2}\left(\Pi_{0}, M\right) .
$$

Proof. The 3-cocycle of $E$ with respect to the unique section system (triv, $\operatorname{id}_{\Pi_{0}}$ ) for $E$ is trivial and hence

$$
\mathrm{H}^{2}(E, M) \cong \operatorname{Hom}_{\Pi_{0}}\left(\Pi_{1}, M\right) \oplus \mathrm{H}^{2}\left(\Pi_{0}, M\right)
$$

by corollary $5.5(\mathrm{~b})(\mathrm{i})$.

Example 5.8. We suppose given a simplicial group $G$ such that $\pi_{1}(G)$ is finite. Then we have

$$
\mathrm{H}^{2}(G, \mathbb{Z}) \cong \mathrm{H}^{2}\left(\pi_{0}(G), \mathbb{Z}\right)
$$

Proof. Since $\pi_{1}(G)$ is finite, we have $\operatorname{Hom}_{\pi_{0}(G)}\left(\pi_{1}(G), \mathbb{Z}\right)=\{0\}$, whence corollary 5.5(a)(ii) applies.

Example 5.9. We suppose given a simplicial group $G$ with $\pi_{0}(G) \cong \pi_{1}(G) \cong \mathrm{C}_{2}$. For $n \in \mathbb{N}_{0}$, we have

$$
\begin{aligned}
\mathrm{H}^{2}(G, \mathbb{Z} / n) \cong & \left.\begin{array}{ll}
\operatorname{Hom}\left(\mathrm{C}_{2}, \mathbb{Z} / n\right) \oplus \mathrm{H}^{2}\left(\mathrm{C}_{2}, \mathbb{Z} / n\right) & \text { if } \mathrm{k}_{G}^{3}=1, \\
\mathrm{H}^{2}\left(\mathrm{C}_{2}, \mathbb{Z} / n\right) & \text { if } \mathrm{k}_{G}^{3} \neq 1,
\end{array}\right\} \\
& \cong \begin{cases}\mathbb{Z} / 2 & \text { if } n=0, \\
\{0\} & \text { if } n \in \mathbb{N}, 2 \nmid n, \\
\mathbb{Z} / 2 \oplus \mathbb{Z} / 2 & \text { if } n \in \mathbb{N}, 2 \mid n, \mathrm{k}_{G}^{3}=1, \\
\mathbb{Z} / 2 & \text { if } n \in \mathbb{N}, 2 \mid n, \mathrm{k}_{G}^{3} \neq 1,\end{cases}
\end{aligned}
$$

where $\mathbb{Z} / n$ is considered as a trivial $\mathrm{C}_{2}$-module.

Proof. The assertion for $\mathrm{k}_{G}^{3}=1$ is a particular case of corollary 5.5(a)(i), so let us suppose that $\mathrm{k}_{G}^{3} \neq 1$. For $n=0$, we get the assertion from example 5.8. So let us suppose given an $n \in \mathbb{N}$. By the additivity of $\mathrm{H}^{2}(G,-)$ resp. $\mathrm{H}^{2}\left(\pi_{0}(G),-\right)$ and the 
Chinese Remainder Theorem, it suffices to consider the case where $n=p^{e}$ for a prime $p$ and $e \in \mathbb{N}$. If $p>2$, we have $\operatorname{Hom}_{\pi_{0}(G)}\left(\pi_{1}(G), \mathbb{Z} / p^{e}\right)=\{0\}$ and hence

$$
\mathrm{H}^{2}\left(G, \mathbb{Z} / p^{e}\right) \cong \mathrm{H}^{2}\left(\pi_{0}(G), \mathbb{Z} / p^{e}\right)
$$

by corollary $5.5(\mathrm{a})(\mathrm{ii})$.

It remains to consider the case $n=2^{e}$ for some $e \in \mathbb{N}$. We let $x$ be the generator of $\pi_{0}(G)$, we let $y$ be the generator of $\pi_{1}(G)$ and we let $z^{3} \in \mathrm{Z}_{\text {cpt }}^{3}\left(\pi_{0}(G), \pi_{1}(G)\right)$ be a componentwise pointed 3 -cocycle with $\mathrm{k}_{G}^{3}=z^{3} \mathrm{~B}_{\mathrm{cpt}}^{3}\left(\pi_{0}(G), \pi_{1}(G)\right)$. Since $\mathrm{k}_{G}^{3} \neq 1$, we have $z^{3} \neq 1$ and hence

$$
(r, q, p) z^{3}= \begin{cases}1 & \text { for }(r, q, p) \neq(x, x, x) \\ y & \text { for }(r, q, p)=(x, x, x) .\end{cases}
$$

Now $\operatorname{Hom}_{\pi_{0}(G)}\left(\pi_{1}(G), \mathbb{Z} / 2^{e}\right)=\operatorname{Hom}\left(\pi_{1}(G), \mathbb{Z} / 2^{e}\right)$ has a unique non-trivial element $z_{1}: \pi_{1}(G) \rightarrow \mathbb{Z} / 2^{e}$, which maps $y$ to $y z_{1}=2^{e-1}$. But for all $c_{0} \in \mathrm{Ch}_{\mathrm{cpt}}^{2}\left(\pi_{0}(G), \mathbb{Z} / 2^{e}\right)$, we have

$$
\begin{aligned}
(x, x, x)\left(c_{0} \partial\right) & =(x, x) c_{0}-(x, 1) c_{0}+(1, x) c_{0}-(x, x) c_{0}=0 \neq 2^{e-1}=y z_{1} \\
& =(x, x, x) z^{3} z_{1} .
\end{aligned}
$$

Hence there does not exist a cochain $c_{0} \in \mathrm{Ch}_{\mathrm{cpt}}^{2}\left(\pi_{0}(G), \mathbb{Z} / 2^{e}\right)$ with $z^{3} z_{1}=c_{0} \partial$. It follows that

$$
\mathrm{Z}_{\mathrm{EM}, z^{3}}^{2}\left(G, \mathbb{Z} / 2^{e}\right)=\{0\} \times \mathrm{Z}_{\mathrm{cpt}}^{2}\left(\pi_{0}(G), \mathbb{Z} / 2^{e}\right)
$$

and thus

$$
\mathrm{H}^{2}\left(G, \mathbb{Z} / 2^{e}\right) \cong \mathrm{H}_{\mathrm{EM}, z^{3}}^{2}\left(G, \mathbb{Z} / 2^{e}\right) \cong \mathrm{H}_{\mathrm{cpt}}^{2}\left(\pi_{0}(G), \mathbb{Z} / 2^{e}\right) \cong \mathrm{H}^{2}\left(\pi_{0}(G), \mathbb{Z} / 2^{e}\right) .
$$

Example 5.10. We consider the crossed module $V$ with group part $\mathrm{Gp} V=\langle a| a^{4}=$ $1\rangle$, module part $\mathrm{Mp} V=\left\langle b \mid b^{4}=1\right\rangle$, structure morphism given by $b \mu=a^{2}$ and action given by ${ }^{a} b=b^{-1}$, cf. [29, ex. (5.6)]. Then we have

$$
\mathrm{H}^{2}(V, \mathbb{Z} / n) \cong \begin{cases}\mathbb{Z} / 2 & \text { for } n \in \mathbb{N}_{0} \text { even }, \\ \{0\} & \text { for } n \in \mathbb{N}_{0} \text { odd }\end{cases}
$$

Proof. The homotopy groups of $V$ are given by $\pi_{0}(V)=\langle x\rangle$ with $x:=a(\operatorname{Im} \mu)$ and $\pi_{1}(V)=\langle y\rangle$ with $y:=b^{2}$, and we have $\pi_{0}(V) \cong \pi_{1}(V) \cong \mathrm{C}_{2}$. Now $\left(s^{1}, s^{0}\right)$ defined by $s^{0}: \pi_{0}(V) \rightarrow \operatorname{Gp} V, 1 \mapsto 1, x \mapsto a$ and $s^{1}: \operatorname{Im} \mu \rightarrow \operatorname{Mp} V, 1 \mapsto 1, a^{2} \mapsto b$ is a section system for $V$. We let $\left(Z^{2}, Z^{1}\right)$ be the lifting system coming from $\left(s^{1}, s^{0}\right)$. It follows that $(x, x) \mathrm{z}^{2}=\left(x s^{0}\right)\left(x s^{0}\right)\left(1 s^{0}\right)^{-1}=a^{2}$ and therefore $(x, x) Z^{2}=a^{2} s^{1}=b$. Finally,

$$
(x, x, x) \mathrm{z}^{3}=(x, x) Z^{2}(1, x) Z^{2}\left((x, 1) Z^{2}\right)^{-1}\left(x Z^{1}(x, x) Z^{2}\right)^{-1}=b^{a}\left(b^{-1}\right)=b^{2}=y
$$

and therefore $\mathrm{z}^{3} \neq 1$. Since

$$
\begin{aligned}
(x, x, x)\left(c^{2} \partial\right) & =(x, x) c^{2}\left((x, 1) c^{2}\right)^{-1}(1, x) c^{2}\left({ }^{x}(x, x) c^{2}\right)^{-1}=(x, x) c^{2}\left((x, x) c^{2}\right)^{-1} \\
& =1
\end{aligned}
$$

for every componentwise pointed 2-cochain $c^{2} \in \mathrm{Ch}_{\text {cpt }}^{2}\left(\pi_{0}(V), \pi_{1}(V)\right)$, we conclude that $\mathrm{z}^{3} \notin \mathrm{B}_{\mathrm{cpt}}^{3}\left(\pi_{0}(V), \pi_{1}(V)\right)$ and hence $\mathrm{k}_{V}^{3} \neq 1$. The assertion follows now from example 5.9 . 


\section{References}

[1] Artin, Michael, Grothendieck, Alexander and Verdier, Jean-Louis. Théorie des topos et cohomologie étale des schémas. Tome 1: Théorie des topos. Lecture Notes in Mathematics, vol. 269. Springer-Verlag, Berlin-New York, 1972. Séminaire de Géométrie Algébrique du Bois-Marie 1963-1964 (SGA4). With the collaboration of N. Bourbaki, P. Deligne and B. Saint-Donat.

[2] Artin, Michael, Grothendieck, Alexander and Verdier, Jean-Louis. Théorie des topos et cohomologie étale des schémas. Tome 2. Lecture Notes in Mathematics, vol. 270. Springer-Verlag, Berlin-New York, 1972. Séminaire de Géométrie Algébrique du Bois-Marie 1963-1964 (SGA4). With the collaboration of N. Bourbaki, P. Deligne and B. Saint-Donat.

[3] Bredon, Glen E. Topology and Geometry. Graduate Texts in Mathematics, vol. 139. Springer-Verlag, New York, 1993.

[4] Brown, Kenneth S. Cohomology of Groups. Graduate Texts in Mathematics, vol. 87. Springer-Verlag, New York-Berlin, 1982.

[5] Brown, Ronald. Groupoids and crossed objects in algebraic topology. Homology, Homotopy and Applications 1 (1999), pp. 1-78.

[6] Brown, Ronald and Spencer, Christopher B. G-groupoids, crossed modules and the fundamental groupoid of a topological group. Indagationes Mathematicae 38(4) (1976), pp. 296-302.

[7] Bullejos, Manuel, Cegarra, Antonio M. and Duskin, John W. Jr. On cat $^{n}$-groups and homotopy types. J. Pure Appl. Algebra 86(2) (1993), pp. 135154.

[8] Bullejos, Manuel, Faro, Emilio and García-Muñoz, Miguel A. Postnikov invariants of crossed complexes. J. Algebra 285(1) (2005), pp. 238-291.

[9] Carrasco, Pilar and Cegarra, Antonio M. Group-theoretic algebraic models for homotopy types. J. Pure Appl. Algebra 75(3) (1991), pp. 195-235.

[10] Cegarra, Antonio M. and Remedios, Josué. The relationship between the diagonal and the bar constructions on a bisimplicial set. Topology Appl. 153(1) (2005), pp. 21-51.

[11] Duskin, John W. Simplicial methods and the interpretation of "triple" cohomology. Mem. Amer. Math. Soc., vol. 3(2), no. 163. Amer. Math. Soc., 1975.

[12] Eilenberg, Samuel and Mac Lane, Saunders. Determination of the second homology and cohomology groups of a space by means of homotopy invariants. Proc. Nat. Acad. Sci. U.S.A. 32 (1946), pp. 277-280.

[13] Eilenberg, Samuel and Mac Lane, Saunders. Cohomology theory in abstract groups. II. Group Extensions with a non-Abelian Kernel. Ann. of Math. 48(2) (1947), pp. 326-341.

[14] Ellis, Graham J. Homology of 2-types. Jour. Lond. Math. Soc. 46(1) (1992), pp. 1-27.

[15] Friedlander, Eric M. and Mazur, Barry. Filtrations on the homology of algebraic varieties. Mem. Amer. Math. Soc., vol. 110, no. 529. Amer. Math. Soc., 1975. 
[16] Goerss, Paul G. and Jardine, John F. Simplicial Homotopy Theory. Progress in Mathematics, vol. 174. Birkhäuser Verlag, Basel, 1999.

[17] Holt, Derek F. An interpretation of the cohomology groups $H^{n}(G, M)$. J. Algebra 60(2) (1979), pp. 307-320.

[18] Huebschmann, Johannes. Crossed $n$-fold extensions of groups and cohomology. Comment. Math. Helv. 55(2) (1980), pp. 302-313.

[19] Jardine, John F. Algebraic Homotopy Theory, Groups, and K-Theory. Ph.D. thesis, The University of British Columbia, 1981.

[20] Kan, Daniel M. On c.s.s. complexes. Amer. J. Math. 79 (1957), pp. 449-476.

[21] Kan, Daniel M. On homotopy theory and c.s.s. groups. Ann. of Math. 68 (1958), pp. 38-53.

[22] Lamotke, Klaus. Semisimpliziale algebraische Topologie. Die Grundlehren der mathematischen Wissenschaften, Band 147. Springer-Verlag, Berlin-New York, 1968.

[23] Loday, Jean-Louis. Spaces with finitely many non-trivial homotopy groups. J. Pure Appl. Algebra 24(2) (1982), pp. 179-202.

[24] Mac Lane, Saunders. Cohomology theory in abstract groups. III. Operator Homomorphisms of Kernels. Ann. of Math. 50(3) (1949), pp. 736-761.

[25] Mac Lane, Saunders. Historical Note. J. Algebra 60(2) (1979), pp. 319-320. Appendix in [17].

[26] May, J. Peter. Simplicial objects in algebraic topology. Chicago Lectures in Mathematics. University of Chicago Press, Chicago (IL), 1992.

[27] Quillen, Daniel G. Homotopical Algebra. Lecture Notes in Mathematics, vol. 43. Springer-Verlag, Berlin-New York, 1967.

[28] Ratcliffe, John G. Crossed extensions. Trans. Amer. Math. Soc. 257(1) (1980), pp. 73-89.

[29] Thomas, Sebastian. (Co)homology of crossed modules. Diploma thesis, RWTH Aachen University, 2007. http://www .math.rwth-aachen.de/ Sebastian. Thomas/publications/

[30] Thomas, Sebastian. The functors $\overline{\mathrm{W}}$ and Diag o Nerve are simplicially homotopy equivalent. J. Homotopy Relat. Struct. 3(1) (2008), pp. 359-378.

[31] Thomas, Sebastian. The third cohomology group classifies crossed module extensions. Manuscript, 2009. arXiv:0911.2861v1 [math.KT].

[32] Weibel, Charles A. An introduction to homological algebra. Cambridge studies in advanced mathematics, vol. 38. Cambridge University Press, Cambridge, 1997.

Sebastian Thomas sebastian.thomas@math.rwth-aachen.de

Lehrstuhl D für Mathematik, RWTH Aachen University, Templergraben 64, D-52062 Aachen, Germany 